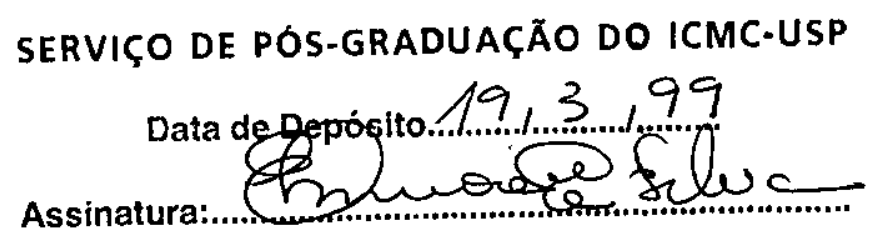

\title{
Os Duais de Hipersuperfícies Genéricas
}

\author{
José Carlos de Souza Junior ${ }^{1}$
}

Orientadora: Profa. Dra. Maria Aparecida Soares Ruas

issertação apresentada ao Instituto de Ciências Matemáticas e de Computação da Universidade de São Paulo, como parte dos requisitos para obtenção do título de "Mestre em Ciências - Área de Matemática".

$$
\text { USP - São Carlos }
$$

Abril - 1999

\footnotetext{
${ }^{1}$ Este trabalho teve suporte financeiro da FAPESP
} 
Aos meus pais José Carlos e Elvira

À minha irmã Maria Cláudia 


\section{Agradecimentos}

Antes de mais nada agradeço a DEUS por ter me dado esta oportunidade.

À toda a minha família por sempre terem dado o seu apoio e em especial aos meus queridos pais.

À minha orientadora, Maria Aparecida Soares Ruas os meus mais sinceros agradecimentos, por ter acreditado no meu trabalho desde o segundo ano da minha graduação e principalmente pela participação efetiva na realização deste projeto de mestrado.

A todos os colegas e funcionários do ICMC-USP.

A todos aqueles que direta ou indiretamente colaboraram para a realização deste trabalho.

À FAPESP 


\section{Resumo}

Neste trabalho estudamos os contatos genéricos de superfícies com famílias de hiperplanos e utilizamos os resultados para descrever os duais destas superfícies e as singularidades da aplicação de Gauss.

Apresentamos duas abordagens para este estudo: o estudo dos teoremas de genericidade para famílias de funções altura definidas em hipersuperfícies em espaços euclidianos, e os correspondentes resultados para hipersuperfícies projetivas. A interpretação geométrica das singularidades destas famílias é discutida nos casos especiais de curvas planas, superfícies em $\mathbb{R}^{3}$ e superfícies em $\mathbb{P}^{3}$. Como aplicação, descrevemos os conjuntos duais nestas dimensões e estudamos as cúspides da aplicação de Gauss projetiva. 


\begin{abstract}
In this work we study the generic contacts of hypersurfaces with families of hyperplanes and we use these results to describe the duals of these surfaces and the singularities of the Gauss map.

We discuss the genericity theorems for the family of height functions of embeddings of hypersurfaces in euclidian spaces and the corresponding results for projective hypersurfaces. The geometric interpretations of the singularities of these families are presented for the cases of plane curves, surfaces in $\mathbb{R}^{3}$ and projective hypersurfaces in $\mathbb{P}^{3}$. As final example, we study the cusps of the projective Gauss map.
\end{abstract}




\section{Sumário}

1 Material Introdutório $\quad 1$

1.1 Germes e jatos ....................... 1

1.2 Equivalência de germes e jatos . . . . . . . . . . . . . 3

1.3 Desdobramentos versais .................. 6

1.4 Transversalidade e genericidade . . . . . . . . . . . . 7

1.5 Classificaçāọ de singularidades . . . . . . . . . . . . . . 9 9

1.6 Classificação dos germes de funções . . . . . . . . . . . . . . 13

1.6.1 Singularidades de Morse e Lema da Decomposição . . . . . . . 13

1.6.2 Singularidades de corank 1 . . . . . . . . . . 15

1.6.3 Singularidades de corank 2 . . . . . . . . . . . 16

1.6.4 Singularidades simples . . . . . . . . . . . . . . 18 
1.6.5 Classificação dos germes $\mathbb{R}^{2}, 0 \rightarrow \mathbb{R}^{2}, 0 \ldots \ldots \ldots \ldots$

2 Duais de hipersuperfícies genéricas em $\mathbb{R}^{n}$

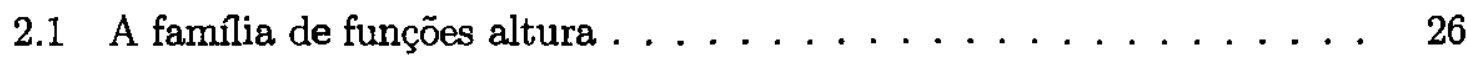

2.2 Curvas planas $\ldots \ldots \ldots \ldots \ldots \ldots \ldots \ldots \ldots \ldots \ldots$

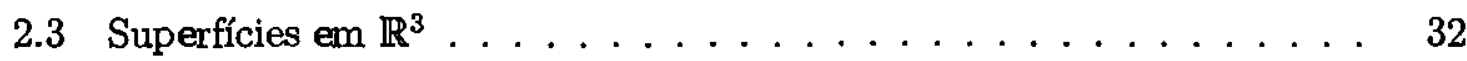

3 Duais de hipersuperfícies projetivas $\quad 39$

3.1 A família de funções altura: definições básicas e resultados preliminares 41

$3.2 \quad$ O teorema de genericidade local $\ldots \ldots \ldots \ldots \ldots$

$3.3 \quad$ O teorema de genericidade multilocal $\ldots \ldots \ldots \ldots \ldots$

3.4 Considerações finais . . . . . . . . . . . . . . . . 65

$\begin{array}{ll}\text { A Geometria Algébrica Projetiva } & 69\end{array}$

A.1 O Espaço Projetivo e as Variedades Projetivas . . . . . . . . . . . 69

A.2 Correspondência entre Ideais Projetivos e Vari- edades Projetivas . . 75

A.3 A Geometria das Hipersuperfícies Quádricas . . . . . . . . . 87 


\section{Introdução}

O objetivo deste trabalho é estudar os contatos genéricos de hipersuperfícies com famílias de hiperplanos e utilizar os resultados para descrever os duais destas superfícies e as singularidades da aplicação de Gauss.

Este estudo é apresentado em duas abordagens: no contexto de subvariedades em espaços euclidianos $\mathbb{R}^{n+1}$ e para hipersuperfícies projetivas em $\mathbb{P}^{n+1}$. A primeira situação tem sido amplamente estudada do ponto de vista da Teoria de Singularidades. O resultado clássico de Looijenga estabelece que para um conjunto residual de mergulhos de subvariedades de dimensão menor ou igual a 5 , as singularidades da família de funções altura são localmente versais. No trabalho, discutimos este teorema e a interpretação geométrica das singularidades da família para curvas planas e superfícies em $\mathbb{R}^{3}$. Os correspondentes teoremas de genericidade para hipersuperfícies projetivas são menos conhecidos e a sua discussão detalhada é a principal parte do trabalho.

No capítulo 1, abordamos noçōes e resultados básicos da teoria de singularidades, como por exemplo, os conceitos de transversalidade e genericidade, métodos de classificação de singularidades e a classificação dos germes simples de funções.

No capítulo 2, estudamos a família de funções altura definida em subvariedades do espaço euclidiano $\mathbb{R}^{n+1}$. Apresentamos o Teorema de Genericidade de Looijenga, e discutimos a maneira de aplicar este resultado ao estudo dos contatos de uma subvariedade genericamente mergulhada com a família de hiperplanos de $\mathbb{R}^{n+1}$. Também analisamos a relação entre as singularidades da família de funçōes altura, a geometria do conjunto dual das hipersuperfícies afins e as singularidades da aplicação normal de Gauss.

A interpretação geométrica das singularidades da família de funções altura é discutida nos casos especiais de curvas em $\mathbb{R}^{2}$ e superfícies em $\mathbb{R}^{3}$. As singulari- 
dades dos conjuntos duais e da aplicação de Gauss são apresentadas.

O capítulo 3 contém a principal parte do trabalho. Nele apresentamos um estudo detalhado dos teoremas 3.2.1, 3.3.1 e 3.3.2, os quais correspondem respectivamente aos teoremas 2.4, 2.8 e 2.10 do artigo "The Duals of Generic Hypersurface", de J. W. Bruce [13], referentes aos contatos genéricos de hipersuperfícies algébricas reais e complexas com famílias de hiperplanos em $\mathbb{P}^{n+1}$. Como aplicação vimos alguns resultados relacionados com as cúspides da aplicação de Gauss projetiva.

O trabalho contém também um apêndice com uma apresentação dos resultados básicos da geometria algébrica projetiva, incluindo em especial um estudo das hipersuperfícies quádricas. A leitura deste apêndice não é essencial para a compreensão do trabalho, mas pode ser útil para uma iniciação à geometria algébrica projetiva. 


\section{Capítulo 1}

\section{Material Introdutório}

Neste capítulo apresentaremos conceitos e resultados básicos em teoria de singularidades que serão necessários para os capítulos seguintes do trabalho. Começaremos apresentando um breve relato sobre germes e jatos, passando então a descrever $\mathcal{A}$ equivalência entre germes. Em seguida voltamos nossa atenção às deformaçōes versais de um germe, e à questão de genericidade. Finalizando, falamos um pouco sobre classificação de singularidades.

\subsection{Germes e jatos}

Sejam $x \in \mathbb{R}^{n}$ e $f, g: U_{1} \subset \mathbb{R}^{n} \rightarrow \mathbb{R}^{p}$ aplicações diferenciáveis definidas em uma vizinhança aberta $U_{1}$ de $x$. Dizemos que $f$ e $g$ são equivalentes e escrevemos $f \sim g$ se existir uma vizinhança $U \ni x$ em $\mathbb{R}^{n}$ tal que $f|U=g| U$, ou seja, se $f$ e $g$ coincidem numa vizinhança $U$ de $x$. Esta relação de equivalência e suas classes de equivalência são chamadas germes de aplicações diferenciáveis de $\mathbb{R}^{n} \rightarrow \mathbb{R}^{p}$ em $x$, 
e os elementos de uma classe são chamados representantes do germe. Se $f$ e $g$ são representantes do mesmo germe então devemos ter $f(x)=g(x)$ e, portanto qualquer outro representante deve assumir o mesmo valor em $x$, digamos $y$. É comum usar-se a notação $f: \mathbb{R}^{n}, x \rightarrow \mathbb{R}^{p}$ para indicar um germe de aplicação.

Consideremos, em particular, os germes em $x$ de aplicações $\mathbb{R}^{n} \rightarrow \mathbb{R}$. Usando as operações da meta $\mathbb{R}$, podemos somar e multiplicar tais aplicações e, assim, vemos que o conjunto de seus germes em $x$ adquire uma estrutura de um anel que denotaremos por $\mathcal{E}_{x}$. Este anel tem um ideal $\mathcal{M}_{x}$ constituido pelos germes de funçōes $\phi: \mathbb{R}^{n}, x \rightarrow$ $\mathbb{R}, 0$, ou seja, funções $\phi \operatorname{com} \phi(x)=0$. Este ideal é um ideal maximal. Na verdade $\mathcal{M}_{x}$ é o único ideal maximal de $\mathcal{E}_{x}$ que é, portanto um anel local. Dado um germe de aplicação $f: \mathbb{R}^{n}, x \rightarrow \mathbb{R}^{p}$ teremos $f=\left(f_{1}, \ldots, f_{p}\right)$ com o $\mathcal{E}_{x}$-módulo (livre) $\mathcal{E}_{p}=$ $\mathcal{E}_{x} \times \ldots \times \mathcal{E}_{x}$ (p cópias). Desta forma, os espaços de germes podem ser identificados com espaços lineares, e considerando-os como variedades podemos identificá-los com seus próprios espaços tangentes.

Vamos agora introduzir uma outra relação de equivalência entre aplicações $\mathbb{R}^{n} \rightarrow$ $\mathbb{R}^{p}$. Sejam $x \in \mathbb{R}^{n}$ e $f, g: U \subset \mathbb{R}^{n} \rightarrow \mathbb{R}^{p}$ aplicações definidas em uma vizinhança $U$ de $x$. Dizemos que $f$ e $g$ têm o mesmo $k$-jato em $x$ se $f(x)=g(x)$ e para algum sistema local de coordenadas em $x \in \mathbb{R}^{n}$ e $f(x) \in \mathbb{R}^{p}$ todas as derivadas parciais de ordem $\leq k$ das componentes de $f$ e $g$ coincidem em $x$. Observe que esta definição depende apenas do germe de $f$ e $g$. Fixando $x \in \mathbb{R}^{n}, y=f(x) \in \mathbb{R}^{p}$ e coordenadas locais em $x \in \mathbb{R}^{n}$ e $y \in \mathbb{R}^{p}$, segue que o $k$-jato de $f$ é determinado pelos termos de grau $\leq k$ nas expansões de Taylor das componentes de $f$ com respeito a estas coordenadas. Estes são os $k$-jatos de aplicações $\mathbb{R}^{n}, 0 \rightarrow \mathbb{R}^{p}, 0$. O conjunto de todos tais $k$-jatos denotase por $J^{k}(n, p)$ o qual é um espaço vetorial de dimensão $p\left(\left(\begin{array}{c}n+k \\ k\end{array}\right)-1\right)$. O conjunto $J^{k}\left(\mathbb{R}^{n}, \mathbb{R}^{p}\right)$ de todos os $k$-jatos (em todos os pontos) de todas as aplicações $\mathbb{R}^{n} \rightarrow \mathbb{R}^{p}$ é um fibrado sobre $\mathbb{R}^{n} \times \mathbb{R}^{p}$ com fibra $J^{k}(n, p)$. Dada então uma aplicação $f: \mathbb{R}^{n} \rightarrow \mathbb{R}^{p}$ temos definida uma aplicação $j^{k} f: \mathbb{R}^{n} \rightarrow J^{k}\left(\mathbb{R}^{n}, \mathbb{R}^{p}\right.$ ) qúe a cada ponto $x \in \mathbb{R}^{n}$ associa 
o $k$-jato de $f$ em $x, j^{k} f(x)$. Esta aplicação é chamada aplicação $k$-jato.

No caso particular em que $p=1$ pode-se mostrar que as derivadas parciais de ordem $\leq k$ de $\phi \in \mathcal{E}_{x}$ se anulam em $x$ se e somente se, $\phi \in \mathcal{M}_{x}^{k+1}$. Assim, podemos identificar o espaço dos $k$-jatos em $x$ de aplicaçōes $\mathbb{R}^{n} \rightarrow \mathbb{R}^{p}$ com o espaço

$$
\frac{\mathcal{E}_{x}}{\mathcal{M}_{x}^{k+1}}
$$

Logo, no caso geral, tem-se

$$
J^{k}(n, p)=\frac{\mathcal{E}_{x}}{\mathcal{M}_{x}^{k+1}} \times \ldots \times \frac{\mathcal{E}_{x}}{\mathcal{M}_{x}^{k+1}}(\mathrm{p} \text { cópias })
$$

\subsection{Equivalência de germes e jatos}

As relaçōes de equivalência fundamentais para o problema de classificação de germes são a $\mathcal{K}$ - e a $\mathcal{A}$ - equivalência, onde $\mathcal{K}$ denota o grupo de contato e $\mathcal{A}$ é o subgrupo de $\mathcal{K}$ de mudanças de coordenadas na fonte e na meta do germe. É este último grupo que nos interessa no momento e é sobre ele que discutiremos a seguir.

Seja $\mathcal{R}=\operatorname{Diff}\left(\mathbb{R}^{n}, x\right)$ o grupo de germes de difeomorfismos $\phi: \mathbb{R}^{n}, x \rightarrow \mathbb{R}^{n}, x$ (ou seja, $\phi \in \mathcal{R}$ é uma mudança de coordenadas locais). Este grupo age sobre $\mathcal{E}_{x}^{p}$ por composição à direita:

$$
\begin{aligned}
& \mathcal{R} \times \mathcal{E}_{x}^{p} \rightarrow \mathcal{E}_{x}^{p} \\
& (\dot{\phi}, f) \mapsto f \circ \phi^{-1}
\end{aligned}
$$

Assim, dizemos que os germes $f, g: \mathbb{R}^{n}, x \rightarrow \mathbb{R}^{p}$ sāo $\mathcal{R}$ - equivalentes, e escrevemos $f \sim_{\mathcal{R}} g$, se existir $\phi \in \mathcal{R}$ tal que $f=g \circ \phi^{-1}$. 
Da mesma forma, o grupo $\mathcal{L}=\operatorname{Diff}\left(\mathbb{R}^{p}, y\right)$ age sobre $\mathcal{E}_{x}^{p}$, agora por composição à esquerda

$$
\begin{aligned}
& \mathcal{L} \times \mathcal{E}_{x}^{p} \rightarrow \mathcal{E}_{x}^{p} \\
& (\psi, f) \mapsto \psi \circ f,
\end{aligned}
$$

e dizemos que dois germes $f, g: \mathbb{R}^{n}, x \rightarrow \mathbb{R}^{p}$ são $\mathcal{L}$ - equivalentes, e escrevemos $f \sim_{\mathcal{L}} g$ se existir $\psi \in \mathcal{L}$ tal que $f=\psi \circ g$. A $\mathcal{R}$ - equivalência (resp. $\mathcal{L}$ - equivalência) é também chamada equivalência à direita (resp. à esquerda), daí o uso da letra $\mathcal{R}$ (abreviação de right em inglês)(resp. $\mathcal{L}$ (abreviação de left em inglês)). O grupo $\mathcal{A}$ de mudanças de coordenadas na fonte e na meta é então definido pelo produto $\mathcal{A}=\mathcal{R} \times \mathcal{L}$ e este grupo age sobre $\mathcal{E}_{x}^{p}$ por composição à direita e à esquerda da seguinte forma:

$$
\begin{aligned}
& \mathcal{A} \times \mathcal{E}_{x}^{p} \rightarrow \mathcal{E}_{x}^{p} \\
& ((\phi, \psi), f) \mapsto \psi \circ f \circ \phi^{-1}
\end{aligned}
$$

Definimos então a seguinte relação de equivalência, chamada de $\mathcal{A}$ - equivalência (ou equivalência à esquerda e à direita). Dois germes $f, g \in \mathcal{E}_{x}^{p}$ são $\mathcal{A}$-equivalentes (ou equivalentes à esquerda e à direita) se existir $(h, k) \in \mathcal{A}$, ou seja, germes de difeomorfismos $h \in \mathcal{R}$ e $k \in \mathcal{L}$ tais que o seguinte diagrama comuta:

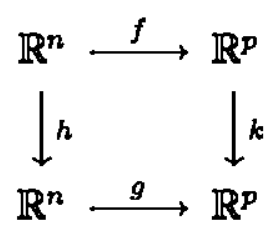

Passamos agora a descrever o espaço tangente à ação do grupo $\mathcal{A}$. Para isto vamos descrever separadamente os espaços tangentes às ações dos grupos $\mathcal{R}$ e $\mathcal{L}$. Começamos com o grupo $\mathcal{R}$. Vamos trabalhar aqui com a fonte e a meta fixas digamos $x=0$ e $y=0$. Neste caso, usamos a notação $\mathcal{E}_{n}$ ao invés de $\mathcal{E}_{x}$ para indicar o anel dos germes de funções $\mathbb{R}^{n}, 0 \rightarrow \mathbb{R}$ e $\mathcal{M}_{n}$ para o respectivo ideal maximal. Dado então um germe $f \in \mathcal{E}_{n}$ seja $\theta(f)$ o conjunto de todos os germes na origem de campos de vetores ao longo de $f$, isto é de germes de aplicações $\zeta$ (como no diagrama abaixo) 
$\operatorname{com} \Pi_{p} \circ \zeta=f$

$$
\begin{array}{ccc}
T\left(\mathbb{R}^{n}\right) & \stackrel{T f}{\longrightarrow} & T\left(\mathbb{R}^{p}\right) \\
\downarrow \Pi_{n} & \nearrow \zeta & \downarrow \Pi_{p} \\
\mathbb{R}^{n} & \stackrel{f}{\rightarrow} & \mathbb{R}^{p}
\end{array}
$$

onde $T\left(\mathbb{R}^{n}\right)$ é o fibrado tangente de $\mathbb{R}^{n}, \Pi_{n}$ é a projeção natural e $T f$ é a aplicação tangente induzida por $f$. Se denotarmos por $\theta(n)$ o conjunto de germes na origem de campos de vetores do $\mathbb{R}^{n}$ (ou seja, campos de vetores ao longo da identidade $\left.I_{n}: \mathbb{R}^{n}, 0 \rightarrow \mathbb{R}^{n}, 0\right)$, um elemento $\chi \in \theta(n)$ será aplicado por $T f$ ao campo $t f(\chi)=$ $T f \circ \chi \in \theta(f)$, e portanto fica definida uma aplicação $t f: \theta(n) \rightarrow \theta(f)$. Observe que $\theta(n)=\theta\left(I_{n}\right)$ é o $\mathcal{E}_{n}$-módulo livre com base $\left\{\partial / \partial x_{1}, \ldots, \partial / \partial x_{n}\right\}$. Logo $t f: \theta(n) \rightarrow$ $\theta(f)$ é a aplicação $\mathcal{E}_{n}$-linear com matriz

$$
\left(\frac{\partial f_{i}}{\partial x_{j}}\right)
$$

Definimos então o espaço tangente à direita de $f$, ou o $\mathcal{R}$-espaço tangente de $f$ como sendo

$$
t f\left(\mathcal{M}_{n} \theta(n)\right)
$$

ou seja, a imagem de $t f$ dos campos de vetores que se anulam na origem. Por outro lado, se $\eta \in \theta(p)$ então a composiçāo $\operatorname{com} f, \eta \circ f \in \theta(f)$, define uma aplicação $w f: \theta(p) \rightarrow \theta(f)$. Também aqui $\theta(p)=\theta\left(I_{p}\right)$ é $\circ \mathcal{E}_{p}$-módulo livre gerado por $\left\{\partial / \partial y_{1}, \ldots, \partial / \partial y_{p}\right\}$, e $w f$ é simplesmente a aplicação induzida por $f^{*}: \mathcal{E}_{p} \rightarrow \mathcal{E}_{n}$, $f^{*}(g)=g \circ f$. Definimos então o espaço tangente à esquerda de $f$ como sendo

$$
w f\left(\mathcal{M}_{p}(\theta(p))\right.
$$

O $\mathcal{A}$-espaço tangente de $f$ é então definido por

$$
T \mathcal{A} f=T \mathcal{R} f+T \mathcal{L} f
$$


Voltemos agora ao caso de jatos. $\mathrm{O} k$-jato de uma composta $h \circ k$ depende somente dos respectivos $k$-jatos das aplicações $h$ e $k$. Assim, as ações de $\mathcal{R}, \mathcal{L}, \mathcal{A}$ sobre o espaço de germes induzem ações dos grupos de Lie (de dimensão finita) $J^{k} \mathcal{R}, J^{k} \mathcal{L}, J^{k} \mathcal{A}$ sobre $J^{k}(n, p)$ (a fibra de $J^{k}\left(\mathbb{R}^{n}, \mathbb{R}^{p}\right)$ sobre o ponto $(0,0)$ ). Como estamos num caso finitodimensional os espaços tangentes a cada uma destas ações podem ser tomados no sentido usual, e apesar de ao nível de germes termos apenas expressōes formais para os espaços tangentes, os respectivos espaços tangentes às órbitas das açōes induzidas são obtidos tomando-se a imagem dos respectivos espaços tangentes ao nível de germes em $\mathcal{M}_{n} \theta(f) / \mathcal{M}_{n}^{k+1} \theta(f)$. Assim, temos

$$
\begin{aligned}
& T \mathcal{R}^{k} f:=T \mathcal{R} f / \mathcal{M}_{n}^{k+1}, \\
& T \mathcal{L}^{k} f:=T \mathcal{L} f / \mathcal{M}_{n}^{k+1}, \\
& T \mathcal{A}^{k} f:=T \mathcal{A} f / \mathcal{M}_{n}^{k+1}
\end{aligned}
$$

Os espaços tangentes definidos acima se referem ao caso em que a fonte e a meta dos germes são fixas (no caso fixamos $x=0$ e $y=0$ ). Se permitirmos que estas variem definimos então os espaços tangentes estendidos por

$$
\begin{aligned}
& T \mathcal{R}_{e} f:=t f(\theta(n)) \\
& T \mathcal{L}_{e} f:=w f(\theta(p)) \\
& T \mathcal{A}_{e} f:=T \mathcal{R}_{e} f+T \mathcal{L}_{e} f
\end{aligned}
$$

\subsection{Desdobramentos versais}

Passamos agora a discutir o conceito de desdobramento de um germe $f$ e, na seção seguinte discutiremos o conceito de genericidade. Estes dois conceitos são de certa forma relacionados um com o outro.

Seja $f: \mathbb{R}^{n}, 0 \times \mathbb{R}^{p}, 0$ um germe diferenciável. Um desdobramento a s parâmetros de $f$ é uma aplicação $F: \mathbb{R}^{n} \times \mathbb{R}^{s}, 0 \rightarrow \mathbb{R}^{p} \times \mathbb{R}^{s}, 0, F(x, \lambda)=(\vec{f}(x, \lambda), \lambda)$ tal que 
$F(x, 0)=(f(x), 0), \forall x$. A aplicação $\bar{f}(x, \lambda)=\bar{f}_{\lambda}(x)$, por sua vez, é denominada uma deformação de $f$. Em outras palavras, um desdobramento de $f$ é simplesmente uma família de germes de aplicações contendo o germe de $f$. Dentre os desdobramentos de um germe $f$ destacam-se os desdobramentos $\mathcal{G}$-versais $(\mathcal{G}=\mathcal{R}, \mathcal{C}, \mathcal{K}, \mathcal{L}, \mathcal{A})$ de $f$, que são de central importância no estudo da geometria do mesmo. Dizemos que um desdobramento $F$ do germe $f$ é $\mathcal{G}$-versal se dado qualquer desdobramento $G(x, u)=(\bar{g}(x, u), u)$ de $f$, para cada parâmetro $u \in \mathbb{R}^{r}$ a aplicação $\bar{g}_{u}: \mathbb{R}^{n}, 0 \rightarrow \mathbb{R}^{p}, 0$, dada por $g_{u}(x)=g(x, u)$, é $\mathcal{G}$-equivalente a $\vec{f}_{\alpha(u)}$ onde $\alpha: \mathbb{R}^{r} \rightarrow \mathbb{R}^{s}$ é uma aplicação diferenciável. Neste caso, dizemos também que $\bar{f}$ é uma deformaçāo versal. O número mínimo $s$ de parâmetros em um desdobramento versal de $F$ é chamado de $\mathcal{G}_{e^{-}}$-codimensão de $f$.Uma deformação versal com o número mínimo de parâmetros é chamada deformação miniversal. Algébricamente os desdobramentos versais são caracterizados pelo seguinte teorema devido a Martinet [1].

Teorema 1.3.1 F: $\mathbb{R}^{n} \times \mathbb{R}^{s}, 0 \rightarrow \mathbb{R}^{p}$ é um desdobramento $\mathcal{G}$-versal $(\mathcal{G}=\mathcal{R}, \mathcal{L}, \mathcal{C}, \mathcal{K}, \mathcal{A})$ do germe $f$ se, e somente se,

$$
T \mathcal{G}_{e} f+\mathbb{R}\left\{\bar{f}_{i}^{\prime}\right\}=\theta(f)
$$

onde $\bar{f}_{i}^{\prime}(x)=\frac{\partial \bar{f}}{\partial u_{i}}(x, 0)$ é a velocidade inicial da deformação $\bar{f}, i=1, \ldots, s$.

Neste trabalho estaremos principalmente interessados nos grupos $\mathcal{G}=\mathcal{A}$ e $\mathcal{R}$.

\subsection{Transversalidade e genericidade}

Fortemente relacionado com o conceito de versalidade está o conceito de genericidade. Dizemos que uma propriedade é genérica em $C^{\infty}(M, N)$ se ela se verifica 
para um conjunto residual de aplicações. Lembremos que um conjunto residual é uma interseção enumerável de abertos densos. Sendo $C^{\infty}(M, N)$ um espaço de Baire, tais conjuntos são densos. A definição mais precisa deste conceito é em termos de resultados de transversalidade, que descrevemos a seguir.

Definição 1.4.1 Sejam $M$ e $N$ variedades diferenciáveis e $f: M \rightarrow N$ uma aplicação $C^{\infty}$. Seja $W$ uma subvariedade de $N$ e $x \in M$. Então $f$ intercepta $W$ transversalmente em $x$ se

(i) $f(x) \notin W$, ou

(ii) $f(x) \in W$ e $T_{f(x)} N=T_{f(x)} W+(d f)_{x}\left(T_{x} M\right)$.

Dizemos que $f$ é transversal a $W$ se $f$ é transversal a $W$ em $x$ para todo $x \in M$.

Lema 1.4.1 (Lema de transversalidade de Thom) Sejam $M, N$ e $P$ variedades diferenciáveis, $W \subset P$ uma subvariedade e $F: N \times M \rightarrow P$ uma aplicação $C^{\infty}$. Se $F$ é transversal a $W$ então para quase todo $y \in M$ a aplicação $F_{y}: N \rightarrow P ; F_{y}(x)=$ $F(x, y)$ é transversal a $W$.

Teorema 1.4.1 (Teorema de transversalidade de Thom) Sejam $Q_{1}, \ldots, Q_{s} s u b$ variedades diferenciáveis do espaço de jatos $J^{k}\left(\mathbb{R}^{n}, \mathbb{R}^{p}\right)$. O conjunto de todas as aplicações diferenciáveis $f: \mathbb{R}^{n} \rightarrow \mathbb{R}^{p}$ para as quais $j^{k} f: \mathbb{R}^{n} \rightarrow J^{k}\left(\mathbb{R}^{n}, \mathbb{R}^{p}\right)$ é transversal a $Q_{1}, Q_{2}, \ldots, Q_{t}$ é denso em $C^{\infty}\left(\mathbb{R}^{n}, \mathbb{R}^{p}\right)$. 


\subsection{Classificação de singularidades}

Quando estudamos a geometria de superfícies é muito comum precisarmos classificar germes de aplicações sob alguma relação de equivalência até uma certa codimensão. Por exemplo, para estudarmos o contato de superfícies em $\mathbb{R}^{3}$ com planos, precisamos de antemão classificar os germes de aplicações $\mathbb{R}^{2}, 0 \rightarrow \mathbb{R}, 0$ de $\mathcal{A}_{e}$-codimensão $\leq 2$. Este processo de classificação envolve basicamente duas etapas: a primeira delas é determinar quais são os "jatos relevantes" em $J^{k}(n, p)$ e então testar se estes jatos são "finitamente determinados" ou não. Este processo é o que chamamos de processo indutivo via jatos, e se baseia em três pontos chaves que são: o conceito de transversal completa introduzido por Bruce, du Plessis e Kirk em [5], o lema de Mather e os teoremas de determinação finita. Destes três pontos o mais conhecido é o lema de Mather que, quando aplicado, reduz os jatos a formas mais simples. Seu enunciado é o seguinte:

Teorema 1.5.1 (Lema de Mather [6]) a) Seja $G$ um grupo de Lie agindo suavemente sobre a variedade $M$ e suponha que a subvariedade $S \subset M$ tem as seguintes propriedades:

1. para cada $x \in S, T_{x} S \subset T_{x} G x$;

2. a dimensão de $G x$ é independente da escolha de $x \in S$;

3. $S$ é uma variedade conexa.

Então $S$ está inteiramente contida em uma única G-órbita.

b) Suponha que $\Pi: M_{1} \rightarrow M_{2}$ é uma $G$-submersão, e seja $S=\Pi^{-1}\left(x_{0}\right)$ para algum $x_{0} \in M_{2}$. Se (1) e (3) de (a) se verificam, então $S$ está contida em uma única G-órbita.

A técnica de classificação, como já dissemos, é o método indutivo de classificação via jatos até que um jato suficiente (e portanto um germe finitamente determinado) 
seja obtido. Comecemos então falando um pouco sobre determinação finita. Dado um grupo $\mathcal{G}$ de equivalências entre germes, dizemos que um germe $f: \mathbb{R}^{n}, 0 \rightarrow \mathbb{R}^{p}, 0$ é $k$ - $\mathcal{G}$-determinado se, para qualquer germe $g$, tal que $j^{k} g(0)=j^{k} f(0)$ tivermos $\mathrm{g}$ $\mathcal{G}$-equivalente a $f$. Dizemos ainda que $f$ é $\mathcal{G}$-finitamente determinado se ele for $k$ determinado para algum $k$. Um $k$-jato é dito suficiente se ele é o $k$-jato de algum germe $k$-determinado. Resultados importantes sobre determinação finita que dão condições necessárias e, em alguns casos, também suficientes para a determinação finita podem ser encontrados em [7]. Um resultado central da teoria é o critério infinitesimal para a determinação finita:

Teorema 1.5.2 ([7]) Seja $\mathcal{G}$ um dos grupos usuais da teoria de singularidades $\mathcal{R}, \mathcal{L}$, $\mathcal{C}, \mathcal{K}, \mathcal{A}$. As seguintes afirmações são equivalentes:

1. $f$ é $\mathcal{G}$-finitamente determinado;

2. para algum $k T \mathcal{G} f \supset \mathcal{M}_{n}^{k} \theta(f)$;

3. para algum $k T \mathcal{G}_{e} f \supset \mathcal{M}_{n}^{k} \theta(f)$;

4. $\operatorname{cod}(f, \mathcal{G})=\operatorname{dim}_{\mathbb{R}}\left\{\mathcal{M}_{\mathbf{n}} \theta(f) / T \mathcal{G} f\right\}<\infty$;

5. $\mathcal{G}_{e}-\operatorname{cod}(f, \mathcal{G})=\operatorname{dim}_{\mathbb{R}}\left\{\theta(f) / T \mathcal{G}_{e} f\right\}<\infty$;

Mais precisamente, pondo $\epsilon=1$ para $\mathcal{G}=\mathcal{R}, \mathcal{C}, \mathcal{K}, e \epsilon=2$ para $\mathcal{L}, \mathcal{A}$, temos:

1. se $f$ é $r$-G-determinado então $T \mathcal{G} f \supseteq \mathcal{M}_{n}^{r+1} \theta(f)$.

2. se $T \mathcal{G} f \supseteq \mathcal{M}_{n}^{r+1} \theta(f)$ entâo $f$ é $(\epsilon r+1)-\mathcal{G}$-determinado.

3. $\operatorname{se} \operatorname{cod}(f, \mathcal{G})=d$ então $T \mathcal{G} f \supseteq \mathcal{M}_{n}^{d+1^{\epsilon}} \theta(f)$.

Alguns resultados de determinação finita utilizam o subgrupo $\mathcal{G}_{1} \subset \mathcal{G}$ de mudanças de coordenadas cujo 1-jato é a identidade. Em geral dado o grupo $\mathcal{G}$ de mudanças de coordenadas, ficam definidos os subgrupos $\mathcal{G}_{s} \subset \mathcal{G}$ de mudanças de coordenadas cujo $s$-jato é a identidade. Nesta direção Bruce, du Plessis e Wall em [8] encontram condições necessárias e suficientes para a determinação finita de germes para uma classe bem abrangente de subgrupos de $\mathcal{K}$, a saber subgrupos unipotentes. $O$ principal 
resultado lá contido é o seguinte teorema, sobre o qual não entraremos em detalhes, por envolver conceitos e técnicas que vão além dos objetivos de nosso trabalho. Antes de enunciarmos o resultado, colocamos um conceito do qual os autores fazem uso no teorema. Diz-se que um subgrupo $\mathcal{H} \subset \mathcal{G}$ é fortemente fechado em $\mathcal{G}$ se $\mathcal{G}_{s} \subset \mathcal{H}$ para algum $s$ e $J^{s} \mathcal{H}$ é um subgrupo fechado de $J^{s} \mathcal{G}$. Se além disso $J^{s} \mathcal{H}$ é um subgrupo algébrico diz-se que $\mathcal{H}$ é fortemente Zariski fechado.

Teorema 1.5.3 ([8]). Seja $\mathcal{G}$ um dos grupos $\mathcal{R}, \mathcal{L}, \mathcal{C}, \mathcal{K}$ ou $\mathcal{A}$. Sejam $\mathcal{H}$ um subgrupo fortemente Zariski-fechado de $\mathcal{G}$ e $f: \mathbb{F}^{n}, 0 \rightarrow \mathbb{F}^{p}, 0$ um germe $C^{r}$ (aqui $\mathbb{F}=\mathbb{R}$ ou $\mathbb{C}$ ); $\tau=\infty$ (aplicações $C^{\infty}$, com $\mathbb{F}=\mathbb{R}$ ) ou $\tau=\mathbb{R}-\omega$ (aplicações analíticas reais), ou $\tau=\mathbb{C}-\omega$ (aplicações analíticas complexas, $\mathbb{F}=\mathbb{C}$ ). Então para qualquer $r<\infty, f$ é $r$-H-determinado se, e somente se, existe um subgrupo de $\mathcal{G}$ fortemente fechado $\mathcal{U} \subset \mathcal{H}$, com $J^{\mathrm{l}} \mathcal{U}$ unipotente para o qual tem-se

$$
\mathcal{M}_{n}^{r+1} \theta(f) \subset T U f
$$

Passamos agora a descrever a outra ferramenta que precisamos no processo de classificação que é o conceito de transversal completa desenvolvido primeiramente por J. W. Bruce e A. A. du Plessis e posteriormente junto com N. Kirk [5]. O resultado central daquele artigo é o seguinte teorema:

Teorema 1.5.4 (Teorema das transversais completas [5]) Seja $\mathcal{H}$ um subgrupo de Lie de $\mathcal{G}_{1}$ e seja $f$ um $k$-jato em $J^{k}(n, p)$. Se $T \subset H^{k+1}(n, p)$ é um subespaço vetorial de $H^{k+1}(n, p)$ tal que

$$
H^{k+1}(n, p) \subset T+T \mathcal{H}^{k+1} f
$$

entäo todo $(k+1)$-jato $F$ com $j^{k} F=f$ é $\mathcal{H}^{k+1}$-equivalente a algum $(k+1)$-jato da forma $f+t$, para algum $f \in T$. 
Bastante útil é a variante deste teorema que se obtem fazendo-se $\mathcal{H}=\mathcal{A}_{1}$.

Proposiçāo 1.5.1 Seja $f$ um $k$-jato no espaço de jatos $J^{k}(n, p)$. Seja ainda $T$ um subespaço vetorial do $H^{k+1}(n, p)$ de jatos homogêneos de grau $k+1$ tal que

$$
H^{k+1}(n, p) \subset T+T \mathcal{A}_{1}^{k+1} f .
$$

Então qualquer $(k+1)$-jato $F$ com $j^{k} F=f$ é $\mathcal{A}_{1}$-equivalente a $f+t$ para algum $t \in T$.

Neste ponto cabe um exemplo para ilustrar o método.

Exemplo 1 Consideremos aqui $\mathcal{A}$-equivalência e aplicações $f: \mathbb{R}^{2}, 0 \rightarrow \mathbb{R}^{2}, 0$ de corank 1 (ver [9]). Um tal germe $f$ deve ter 1-jato da forma $\sigma=(x, 0)$. Vamos determinar uma 2-transversal completa para $\sigma$. Como podemos ver facilmente, os geradores do espaço tangente $T \mathcal{A}_{1}^{2} \sigma$ são $\left(x^{2}, 0\right),(x y, 0),\left(y^{2}, 0\right),\left(0, x^{2}\right)$. Assim, podemos tomar como subespaço $T$ no teorema 1.5 .4 o subespaço de $H^{2}(2,2)$ gerado pelos vetores $(0, x y),\left(0, y^{2}\right)$. Portanto de acordo com o mesmo teorema qualquer 2-jato sobre $(x, 0)$ é $\mathcal{A}$-equivalente $a\left(x, a_{1} x y+a_{2} y^{2}\right)$, com $a_{1}, a_{2} \in \mathbb{R}$. Teremos então 3 casos a considerar: $a_{2} \neq 0 ; a_{2}=0$ com $a_{1} \neq 0$; ou $a_{1}=a_{2}=0$. Chegamos então aos três possiveis 2-jatos que são $\left(x, y^{2}\right),(x, x y),(x, 0)$. O primeiro deles é $2-\mathcal{A}_{1}$ determinado. Poderíamos agora continuar, por exemplo, a classificar os germes sobre o 2-jato $(x, x y)$ ou $(x, 0)$ em codimensões baixas. Por exemplo, um dos três jatos sobre $(x, 0)$ é $\left(x, y^{3}\right)$. Pensando este 3 -jato como um $k$-jato (com $k \geq 3$ ) e analisando o seu espaço tangente $T \mathcal{A}_{1}^{k+1} f$, vemos que o único termo de grau $k+1$ que não está presente é o vetor $\left(0, x^{k} y\right)$. Logo uma transversal completa em $J^{k+1}(2,2)$ é dada por $\left(x, y^{3}+a x^{k} y\right)$, com $a \in \mathbb{R}$. Se a $\neq 0$ com mudanças de escalas na fonte e na meta podemos reduzíla às formas normais $\left(x, y^{3} \pm x^{k} y\right)$ para $k$ par $e\left(x, y^{3}+x^{k} y\right)$ para $k$ impar. Estes germes são $\mathcal{A}$-finitamente determinados ([9]) e, portando, temos uma lista completa dos germes $\mathcal{A}$-finitamente determinados com 3-jato $\left(x, y^{3}\right)$. 
$\mathrm{Na}$ seçāo seguinte listamos alguns resultados importantes de classificação, dos quais faremos uso nos capítulos seguintes.

\subsection{Classificação dos germes de funções}

Seja $f: \mathbb{R}^{n}, 0 \rightarrow \mathbb{R}, 0$ um germe de uma função suave. Se $\nabla f(0) \neq 0$, então $f \simeq_{\mathcal{R}} x_{1}$. Se $f$ é singular em 0 , o objetivo é determinar uma lista de singularidades finitamente determinadas. É claro que não podemos obter todos os germes finitamente determinados, portanto, restringimo-nos ao caso dos germes simples.

A classificação dos germes de funções (ou de aplicações) é obtida por indução sobre o espaço de $k$-jatos. Dado um $j^{k} f$ usamos o teorema da Transversal Completa para obter uma parametrização dos $(k+1)$-jatos que tem $\circ k$-jato igual a $j^{k} f$. Podemos usar o Lema de Mather para produzir as órbitas dentro desta parametrização. Aplicamos o teste da determinação finita a cada órbita no $(k+1)$-jato (cuja $k$-jato é $j^{k} f$ ). Se o germe é finitamente determinado paramos o processo. Se não, consideramos $\circ(k+2)$ jato. No caso de funções precisamos dos resultados que apresentaremos na sequência, e cujas principais referências são [3] e [12]

\subsubsection{Singularidades de Morse e Lema da Decomposição}

Definiçāo 1.6.1 Um germe $f \in m_{n}$ é dito de Morse se $j^{2} f$ é uma forma quadrática não degenerada.

Lema 1.6.1 Um germe de Morse é $\mathcal{R}$-equivalente a $\sum_{i=1}^{n} \pm x_{i}^{2}$. 
Seja $f \in m_{n}$, então o $\mathcal{R}$-espaço tangente estendido de $f$ é dado por $T \mathcal{R}_{e} . f=$ $J(f)$, onde $J(f)$ denota o ideal Jacobiano de $f$. Segue do teorema fundamental dos desdobramentos versais (ver Teorema 1.3.1) que um desdobramento de $f$ é $\mathcal{R}_{e}$-versal se, e somente se,

$$
J(f)+\mathbb{R}\left\{\dot{F}_{1}, \cdots, \dot{F}_{s}\right\}=\mathcal{E}_{n} .
$$

Isto implica na seguinte proposição.

Proposição 1.6.1 Uma deformação $\mathcal{R}_{e}$-miniversal de um germe de Morse $f$ é dada por $f(x, u)=f(x)+u$.

Observação 1.6.1 No caso do grupo $\mathcal{K}$ ou $\mathcal{A}$ um germe de Morse é estável.

Definição 1.6.2 Um germe $f \in m_{n}^{2}$ tem corank $q$ se a matriz Hessiana $\left(\partial^{2} f / \partial x_{i} \partial x_{j}(0)\right)$ tem rank $p=n-q$.

Lema 1.6.2 ("Splitting Lemma"ou Lema de Decomposição) Se $f \in m_{n}^{2}$ é uma singularidade de corank $q$, então $f$ é $\mathcal{R}$-equivalente a um germe da forma

$$
Q\left(x_{1}, \cdots, x_{p}\right)+g\left(x_{p+1}, \cdots, x_{n}\right)
$$

onde $p+q=n, Q$ uma forma quadrática não degenerada e $j^{2} g=0$.

Demonstração: Podemos supor que $j^{2} f$ é dado por uma forma quadrática não degenerada $Q\left(x_{1}, \cdots, x_{p}\right)$, e considerar $f$ como um desdobramento de $Q$. Como $Q+u$ é miniversal, segue que $f$ é isomorfo a $g^{*}(Q+u)$ por algum germe $g: \mathbb{R}^{n-p}, 0 \rightarrow \mathbb{R}, 0$. Isto é, existe um difeomorfismo $\phi$ tal que

$$
f\left(\phi\left(x_{1}, \cdots, x_{n}\right)\right)=Q\left(x_{1}, \cdots, x_{p}\right)+g\left(x_{p+1}, \cdots, x_{n}\right) .
$$


Observemos que a singularidade de $f$ é completamente determinada pela singularidade de $g$. A vantagem do uso do Lema de Decomposição é que $g$ tem um número menor de variáveis. Podemos classificar agora os germes de funçōes usando o Teorema da Transversal Completa e o Teorema 1.5.1 da determinação finita, a saber, se

$$
m_{n}^{k} \cdot \mathcal{E}_{n} \subset T \mathcal{R} . f+m_{n}^{k+1} \cdot \mathcal{E}_{n}
$$

então $f$ é $k-\mathcal{R}$-determinado.

\subsubsection{Singularidades de corank 1}

Usando o Lema de Decomposiçāo, temos $f=g(x)+q\left(x_{2}, \cdots, x_{n}\right)$, onde $q$ é uma forma quadrática não degenerada. Então o problema neste caso se reduz a classificar funçōes de uma só variável.

Se $g(0)=\cdots=g^{(k)}=0$ mas $g^{(k+1)} \neq 0$, então não é difícil mostrar que $g$ é $(k+1)$-determinado e, portanto,

$$
g \simeq_{\mathcal{R}} \pm x^{k+1}
$$

A singularidade $\pm x^{k+1}$ é chamada $A_{k}$. Ela é de $\mathcal{R}_{e}$-codimensão $k$. Um desdobramento versal é dado por

$$
\pm x^{k+1}+u_{k-1} x^{k-1}+\cdots+u_{1} x+u_{0}
$$

A constante $u_{0}$ representa os níveis de $f_{u}(x)$. Em alguns casos somente a singularidade de $f$ é de interesse. Neste caso, é definido o desdobramento versal "potencial"como um desdobramento que satisfaz

$$
J(f)+\mathbb{R}\left\{\dot{F}_{1}, \cdots, \dot{F}_{s}\right\}=m_{n} \cdot \mathcal{E}_{n} .
$$


No caso da singularidade $\pm x^{k+1}$, um modelo de um desdobramento potencial é $\pm x^{k+1}+u_{k-1} x^{k-1}+\cdots+u_{1} x$. Uma outra maneira de eliminar o termo constante é considerar o grupo $\mathcal{K}$ ou $\mathcal{A}$. Nestes casos podemos obter o termo constante no desdobramento usando o grupo $\mathcal{L}$.

\subsubsection{Singularidades de corank 2}

Pelo Lema de Decomposição temos que considerar germes de funções de duas variáveis $g(x, y)$ com $j^{2} g=0$.

Seja $j^{3} g=a_{0} x^{3}+a_{1} x^{2} y+a_{2} x y^{2}+a_{3} y^{3}$. Podemos mudar variáveis e escrever $g$ na forma

$$
g \simeq_{\mathcal{R}} \begin{cases}x^{3} \pm x y^{2} & \text { se } g \text { possui uma ou três raízes reais } \\ x y^{2} & \text { se } g \text { possui uma raiz dupla } \\ x^{3} & \text { se } g \text { é uma cúbica perfeita } \\ 0 & \text { se } g=0\end{cases}
$$

O 3-jato $x^{3} \pm x y^{2}$ é 3-determinado pois

$$
m^{3}(x, y) \subset m(x, y)<3 x^{2} \pm y^{2}, \pm 2 x y>+m^{4}(x, y)
$$

Esta singularidade é chamada $D_{4}$ e tem $\mathcal{R}_{e}$-codimensão 4 . Um desdobramento versal é dado por

$$
x^{3} \pm x y^{2}+u_{3} x^{2}+u_{2} x+u_{1} y+u_{0}
$$

Como no caso anterior, não precisamos do termo $u_{0}$ no caso de um $\mathcal{A}$-desdobramento.

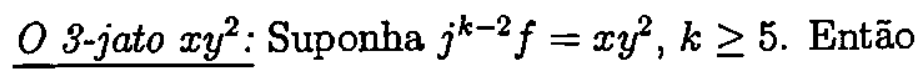

$$
J^{k-1}\left(m^{2}(x, y)<y^{2}, 2 x y>\right) \cap H^{k-1}(x, y)+\mathbb{R} .\left\{x^{k-1}\right\}=H^{k-1}(x, y)
$$


Segue do teorema da Transversal Completa que todos os $(k-1)$-jatos cujos $(k-2)$ jatos são iguais a $x y^{2}$ são equivalentes a $x y^{2}+a x^{k-1}$ para algum $a \in \mathbb{R}$. Uma mudança de coordenadas mostra que as órbitas nesta familia são

$$
x y^{2} \pm x^{k-1} \text { e } x y^{2}
$$

A singularidade $x y^{2} \pm x^{k-1}$ é $(k-1)$-determinada, pois

$$
m^{k-1}(x, y) \subset m(x, y)<y^{2} \pm(k-1) x^{k-2}, 2 x y>+m^{k}(x, y) .
$$

Esta singularidade é chamada $D_{k}$ e tem $\mathcal{R}_{e}$-codimensão $k$. Um desdobramento versal é dado por

$$
\begin{gathered}
x y^{2} \pm x^{k-1}+u_{k-1} x^{k-2}+\cdots+u_{2} x+u_{1} y+u_{0} . \\
\text { O 3-jato } x^{3}: \text { Temos } f_{x}=3 x^{2} \text { e } f_{y}=0, \\
J^{4}\left(m^{2}(x, y)<3 x^{2}, 0>\right) \cap H^{4}(x, y)+\mathbb{R} .\left\{x y^{3}, y^{4}\right\}=H^{4}(x, y) .
\end{gathered}
$$

Então um elemento $g$ de $J^{4}(2,1) \operatorname{com} j^{3} g=f$ é equivalente a $x^{3}+a x y^{3}+b y^{4}$ para algun par $a, b \in \mathbb{R}$.

- Se $b \neq 0$, então $g \simeq_{\mathcal{R}} x^{3}+a x y^{3} \pm y^{4}$. Seja $V=\left\{x^{3}+a x y^{3} \pm y^{4}, a \in \mathbb{R}\right\}$ um espaço vetorial em $M=J^{4}(2,1)$ e seja $G=J^{4} \mathcal{R}$. Aplicando o Lema de Mather segue que $V$ está contido em uma só órbita, o que mostra que $g \simeq_{\mathcal{R}} x^{3} \pm y^{4}$. Esta singularidade é 4-determinada e é denotada por $E_{6}$. Ela tem $\mathcal{R}_{e}$-codimensão $6 \mathrm{com}$ um desdobramento versal dado por

$$
x^{3} \pm y^{4}+u_{5} x y^{2}+u_{4} y^{2}+u_{3} x y+u_{2} x+u_{1} y+u_{0} .
$$

- Se $b=0$ e $a \neq 0$, então $g \simeq_{\mathcal{R}} x^{3}+x y^{3}$. Esta singularidade é 5 determinada e é denotada por $E_{7}$. Tem $\mathcal{R}_{e}$-codimensão $7 \mathrm{com}$ um desdobramento versal dado por

$$
x^{3}+x y^{3}+u_{6} y^{4}+u_{5} y^{3}+u_{4} x y^{2}+u_{3} y^{2}+u_{2} x y+u_{1} x+u_{0} .
$$


- Se $a=b=0$, então um 5-jato cujo 4-jato é $x^{3}$ é equivalente a $x^{3}+c x y^{4}+d y^{5}$. Se $d \neq 0$, então $g \simeq x^{3}+y^{5}$. Esta singularidade, denotada por $E_{8}$, é 5-determinada e tem $\mathcal{R}_{e}$-codimensāo 8 . Um desdobramento versal é dado por

$$
x^{3}+y^{5}+u_{7} x y^{3}+u_{6} y^{3}+u_{5} x y^{2}+u_{4} y^{2}+u_{3} x y+u_{2} x+u_{1} y^{4}+u_{0}
$$

\subsubsection{Singularidades simples}

Definição 1.6.3 Uma singularidade finitamente determinada $f \in m_{n} \mathcal{E}(n, p)$ é dita $\mathcal{G}$-simples se existe uma vizinhaça $V$ de $j^{k} f$ ( $k$ grande) em $J^{k}(n, p)$ tal que $V$ contém um número finito de órbitas de $J^{k} \mathcal{G}$ em $J^{k}(n, p)$.

Exemplo 2 (1) $n=p=1, f(x)= \pm x^{k+1}$ é $\mathcal{R}$-simples, pois para $l \geq k+1$, uma vizinhança de $j^{l} f$ que contém só as órbitas $A_{s}, s \leq k$. (Ver o desdobramento versal $\left.\operatorname{de} A_{k}\right)$.

(2) Nem todos os germes sāo $\mathcal{R}$-simples. Seja

$$
f_{t}(x, y)=x y(x+y)(x-t y)
$$

O conjunto $f^{-1}(0)$ é formado por 4 retas passando pelo origem. Podemos mostrar que $f_{t}$ é finitamente determinado mas $f_{t}$ e $f_{t^{\prime}}$ não são $\mathcal{R}$-equivalentes para $t \neq t^{\prime}$.

Proposição 1.6.2 Se $f \in m_{n}$ é $\mathcal{R}$-simples, então corank $(f) \leq 2$.

Demonstração: $j^{2} f$ define uma forma quadrática de rank $r$ em $\mathbb{R}^{2}$. O espaço nulo desta forma quadrática é um subespaço vetorial de dimensão $n-r . j^{3} f$ define uma forma cúbica em $V$ que é independente do sistema de coordenadas. Se $\phi \in \mathcal{R}$ entāo 
$D \phi(0)$ leva $V_{j^{2} f}$ a $V_{j^{2}(f \circ \phi)}$. Então a ação de $J^{3} \mathcal{R}$ sobre $J^{3}(n, 1)$ induz uma ação de $G L(n-r, \mathbb{R})$ sobre o espaço das formas cúbicas de $(n-r)$-variáveis. Assim, se as formas cúbicas definidas pelos germes $f, g$ de rank $r$ não são $G L(n-r, \mathbb{R})$-equivalentes, então $f$ e $g$ não são $\mathcal{R}$-equivalentes.

O espaço de cúbicas de $(n-r)$-variáveis é de dimensão $\left(\begin{array}{c}n-r+2 \\ 3\end{array}\right)$. Mas $\operatorname{dim} G L(n-r, \mathbb{R})=(n-r)^{2} ;$ logo se $n-r \geq 3$, então $\left(\begin{array}{c}n-r+2 \\ 3\end{array}\right)>(n-r)^{2}$, e portanto as 6rbitas de $G L(n-r, \mathbb{R})$ têm codimensão $>1$. Isto é qualquer vizinhança encontra um número infinito de órbitas.

O número $\mathcal{R}_{e}-\operatorname{cod}(f, \mathcal{R})=\operatorname{dim}_{\mathbb{R}}\left\{\theta(f) / T \mathcal{R}_{e} f\right\}$ é um importante invariante no estudo das singularidades de funções e é chamado o número de Milnor de $f$ e denotado por $\mu(f)$, ou simplesmente $\mu$.

Teorema 1.6.1 (Arnold [10]) As singularidades $\mathcal{R}$-simples em $m_{n}$ são:

$$
\begin{aligned}
& A_{k}, k \geq 1 \text { cuja forma normal é } \pm x^{k+1} \text { e } \mu=k \\
& D_{k}, k \geq 4 \text { cuja forma normal é } x y^{2} \pm x^{k-1} \text { e } \mu=k \\
& E_{6} \text { cuja forma normal é } x^{3} \pm y^{4} \text { e } \mu=6 \\
& E_{7} \text { cuja forma normal é } x^{3} \pm x y^{3} \text { e } \mu=7 \\
& E_{8} \text { cuja forma normal é } x^{3}+y^{5} \text { e } \mu=8
\end{aligned}
$$

Demonstração: Usando os desdobramentos versais das singularidades no teorema, podemos mostrar que estas singularidades são simples. Segue da Proposição 1.6.2 que só precisamos considerar os germes de corank $\leq 2$. 
Se corank $(f)=1$, então $f \simeq_{\mathcal{R}} \pm x^{k+1}$ que é simples.

Se corank $(f)=2$, então se $f$ tem uma singularidade $D_{k}, E_{6}, E_{7}$ ou $E_{8}, f$ é simples. Caso contrário, usando o Lema de Mather podemos mostrar fácilmente que $f$ não é simples.

Podemos agora desenhar o diagrama de adjacências das singularidades simples:

$$
\begin{aligned}
& A_{1} \hookleftarrow A_{2} \leftarrow A_{3} \longleftarrow A_{4} \longleftarrow A_{5} \longleftarrow A_{6} \longleftarrow A_{7} \longleftarrow A_{8} \cdots \\
& \nwarrow \nwarrow \uparrow \nwarrow \uparrow \nwarrow \uparrow \nwarrow \\
& D_{4} \longleftarrow D_{5} \longleftarrow D_{6} \longleftarrow D_{7} \longleftarrow D_{8} \\
& E_{6} \longleftarrow E_{7} \longleftarrow E_{8}
\end{aligned}
$$

Observação 1.6.2 1. A relação entre as singularidades simples e as álgebras de Lie simples designadas pelos mesmos nomes ou grupos gerados pelas reflexões é estudada em /V.I. Arnold, Remarks on the stationary phase methods and the Coxeter numbers, Russian Maths. Surveys,28:5, 19-48].

2. Um germe de função é $\mathcal{A}$-simples se, somente se, é $\mathcal{K}$-simples se, somente se, é $\mathcal{R}$-simples.

\subsubsection{Classificação dos germes $\mathbb{R}^{2}, 0 \rightarrow \mathbb{R}^{2}, 0$}

A teoria de singularidades começou com os trabalhos de Whitney (1955) sobre a classificação de singularidades estáveis do plano no plano. Muitas listas de germes $\mathbb{R}^{2}, 0 \rightarrow \mathbb{R}^{2}, 0$ seguiram, a mais longa até hoje é aquela apresentada por Rieger [g]. 
Daremos uma classificação dos germes de corank 1 e codimensão $\leq 2$ usando a técnica da Transversal Completa.

Seja $F: \mathbb{R}^{2}, 0 \rightarrow \mathbb{R}^{2}, 0$ um germe de uma aplicação suave. Se corank $(F) \leq 1$, então podemos mudar coordenadas e escrever $F$ na forma

$$
F(x, y)=(x, f(x, y))
$$

Pretendemos classificar germes sob a ação do grupo $\mathcal{A}$ que tem $\mathcal{A}_{e}-\operatorname{cod} \leq 2$. Seguimos o mesmo método da classsificação de funções, a saber classificar pela indução sobre os espaços de jatos.

Denotamos por $a_{k, i}$ o coeficiente do monômio $x^{k-i} y^{i}$ na serie de Taylor de $f$.

Se $a_{1,1} \neq 0$ então $F$ é um germe de um difeomorfismo, portanto $F \simeq_{\mathcal{A}}(x, y)$. Assim, suponhamos que $a_{1,1}=0$.

Lema 1.6.3 As $J^{2} \mathcal{A}$-órbitas singulares em $J^{2}(2,2)$ são $\left(x, y^{2}\right),(x, x y)$ e $(x, 0)$.

Demonstração: $j^{2} f=\left(x, a_{1,0} x+a_{2,0} x^{2}+a_{2,1} x y+a_{2,2} y^{2}\right)$. Podemos eliminar o termo $a_{1,0}$ usando a ação à esquerda. Se $a_{2,2} \neq 0$, então a mudança $y \mapsto y-\frac{a_{2,1}}{2 a_{2,2}} x$ elimina o termo xy. Para eliminar o termo $x^{2}$, usamos uma mudança de variáveis na meta da forma $(u, v) \mapsto\left(u, v-\alpha u^{2}\right)$. O germe final é da forma $\left(x, a_{2,2} y^{2}\right)$. Uma mudança escalar reduz ao germe $\left(x, y^{2}\right)$.

Se $a_{2,2}=0$ e $a_{2,1} \neq 0$, então a mudança $Y=a_{2,0} x+a_{2,1} y$, reduz $a(x, x y)$.

Se $a_{2,2}=a_{2,1}=0$, então. $j^{2} f \simeq(x, 0)$.

Proposição 1.6.3 O germe $F(x, y)=\left(x, y^{2}\right)$ é 2-A-determinado e é $\mathcal{A}_{e}$ - estável. 


\section{Classificação dos germes com o 2-jato igual a $(x, x y)$}

Considere um $k$-jato cujo 2-jato é $(x, x y)$. Usando uma mudança de coordenadas da forma $Y=y-p(x, y)$ podemos eliminar todos os termos divisiveis por $x$ na segunda componente exceto xy. Então este $k$-jato é equivalente $a\left(x, x y+\sum_{i=3}^{k} a_{i, i} y^{i}\right)$.

Proposição 1.6.4 Os germes de $\mathcal{A}_{\boldsymbol{e}}-\operatorname{cod} \leq 2$ com o 2-jato igual a $(x, x y)$ são equivalentes a um dos seguintes germes

$\begin{array}{llr}\text { Cúspide } & \left(x, x y+y^{3}\right) & \mathcal{A}_{e}-c o d=0 \\ \text { Rabo de andorinha } & \left(x, x y+y^{4}\right) & 1 \\ \text { Borboleta } & \left(x, x y+y^{5}+y^{7}\right) & 2\end{array}$

Classificação dos germes com o 2-jato igual a $(x, 0)$

Lema 1.6.4 As $J^{3} \mathcal{A}$-órbitas singulares em $J^{3}(2,2)$ que têm o 2-jato equivalente a $(x, 0) s \tilde{a} o\left(x, y^{3} \pm x^{2} y\right),\left(x, y^{3}\right),\left(x, x y^{2}\right) e(x, 0)$.

Demonstraçāo: Seja $J^{3} F=\left(x, a_{3,0} x^{3}+a_{3,1} x^{2} y+a_{3,2} x y^{2}+a_{3,3} y^{3}\right)$.

- Se $a_{3,3} \neq 0$, então usando a mudança $Y=y-\frac{a_{3,2}}{3 a_{3,3}} x$ e eliminando o termo $x^{3}$ por uma mudança de coordenadas na meta, reduzimos $j^{3} F$ a $\left(x,\left(3 a_{3,1} a_{3,3}-a_{3,2}^{2}\right) / a_{3,3} x^{2} y+\right.$ $\left.a_{3,3} y^{3}\right)$.

Se $3 a_{3,1} a_{3,3}-a_{3,2}^{2} \neq 0$, então uma mudança escalar reduz $a\left(x, y^{3} \pm x^{2} y\right)$. Se não, $j^{3} F \simeq\left(x, y^{3}\right)$.

- Se $a_{3,3}=0$ e $a_{3,2} \neq 0$, então usando o Lema de Mather podemos mostrar que $j^{3} F \simeq\left(x, a_{3,2} x y^{2}\right) \simeq\left(x, x y^{2}\right)$. 
- Se $a_{3,3}=a_{3,2}=0$ e $a_{3,1} \neq 0$, então usando o Lema de Mather $j^{3} F \simeq\left(x, x^{2} y\right)$.

- Se todos os coeficientes se anulam, então $j^{3} F=(x, 0)$.

Proposição 1.6.5 Um $(k+1)$-jato cujo $k$-jato $(k \geq 3)$ é equivalente a $\left(x, y^{3}\right)$ é equivalente a $\left(x, y^{3} \pm x^{k} y\right)$ ou $\left(x, y^{3}\right)$. O germe $\left(x, y^{3} \pm x^{k} y\right)(k \geq 2)$ é $(k+1)$ determinado é tem $\mathcal{A}_{e}-$ cod igual a $(k-1)$.

Podemos mostrar que um $k$-jato cujo $(k-1)$-jato $(k \geq 3)$ é equivalente a $\left(x, x y^{2}\right)$ é equivalente a $\left(x, x y^{2}+\sum_{i=4}^{k} a_{i, i} y^{i}\right)$. Se $a_{4,4} \neq 0$, então o 4 -jato é equivalente $a$ $\left(x, x y^{2}+y^{4}\right)$.

Proposição 1.6.6 As órbitas finitamente determinadas da "raiz" $\left(x, x y^{2}+y^{4}\right)$ são dadas por $\left(x, x y^{2}+y^{4}+y^{2 k+1}\right), k \geq 2$. Este germe é $(2 k+1)$-determinado e tem $\mathcal{A}_{e}-$ cod igual a $k-2$.

Observação 1.6.3 Os demais germes conduzem a $k$-jatos de codimensão $>2$.

Em conclusão temos

Proposição 1.6.7 Os germes de codimensão $\leq 2$ de aplicações $\mathbb{R}^{2}, 0 \rightarrow \mathbb{R}^{2}, 0$ e seus desdobramentos $\mathcal{A}_{e}$-versais são os seguintes: 
Nome

Submersão

Dobra

Cúspide

Rabo de andorinha

Lábios/Bicos

Borboleta

Ganso

Gaivota

$$
(x, y)
$$$$
\left(x, y^{2}\right)
$$$$
\left(x, x y+y^{3}\right)
$$$$
\left(x, x y+y^{4}\right)
$$$$
\left(x, y^{3} \pm x^{2} y\right)
$$$$
\left(x, x y+y^{5} \pm y^{7}\right)
$$$$
\left(x, y^{3}+x^{3} y\right)
$$$$
\left(x, x y^{2}+y^{4}+y^{5}\right)
$$

$(x, y)$

$\left(x, y^{2}\right)$

$\left(x, x y+y^{3}\right)$

$\left(x, x y+y^{4}+u y^{2}\right)$

$\left(x, y^{3} \pm x^{2} y+u y\right)$

$\left(x, x y+y^{5}+y^{7}+u y^{2}+v y^{3}\right)$

$\left(x, y^{3}+x^{3} y+u y+v x y\right)$

$\left(x, x y^{2}+y^{4}+y^{5}+u y+v y^{3}\right)$
0

0

0

1

1

2

2

2 


\section{Capítulo 2}

\section{Duais de hipersuperfícies genéricas em $\mathbb{R}^{n}$}

A teoria de singularidades não somente reinterpreta resultados clássicos da geometria diferencial local das subvariedades em espaços euclidianos, como também permite a descoberta de novos resultados sobre esta geometria. Os novos resultados seguiram da sugestâo de René Thom que consiste em estudar o contato das superfícies com conjuntos especiais tais como planos, linhas, esferas, círculos, etc.. Uma resenha dos resultados nesta área nos ultimos 20 anos é feita por J.W.Bruce em [16].

Neste capitulo, estudamos os contatos genéricos de subvariedades de $\mathbb{R}^{n}$ com familias de hiperplanos. A referência principal para este estudo é o artigo The duals of generic hypersurfaces de J.W. Bruce [13]. 


\subsection{A família de funções altura}

Seja $S^{n}=S$ a esfera unitária em $\mathbb{R}^{n+1}$ e seja $N$ uma variedade diferenciável. Dado um mergulho $f: N \rightarrow \mathbb{R}^{n+1}$, considere a familia de funções

$$
N \times S \stackrel{H_{f}}{\rightarrow} \mathbb{R} \times S \stackrel{\pi}{\rightarrow} S \text { onde } H_{f}(x, u)=(f(x) \cdot u, u), \pi(c, u)=u
$$

para $(x, u, c) \in N \times S \times \mathbb{R}$ e o "ponto" denota o produto interno usual em $\mathbb{R}^{n+1}$.

A familia $H_{f}$ chamada "familia de funções altura" associada a $f$, descreve os contatos de $N$ com a familia de hiperplanos ortogonais a $u \in S$. Quando não houver perigo de confusão, omitiremos o índice $f$. Para cada $u \in S$, indicaremos por $H_{u} a$ aplicação

$$
\begin{aligned}
& H_{u}: N \rightarrow \mathbb{R} \\
& x \longmapsto H_{u}(x)=H(x, u)
\end{aligned}
$$

$O$ conjunto dos pontos críticos $\Sigma H$ de $H$ é o conjunto $\{(x, u) ; x$ é um ponto crítico de $\left.H_{u}: N \rightarrow \mathbb{R}\right\}$, que é o fibrado normal unitário a $N$.

Quando $N$ é compacta de dimensão $n, N$ separa $\mathbb{R}^{n+1}$ e a projeção $\pi_{1}: \Sigma H \rightarrow N$ é um recobrimento duplo, cada uma das folhas correspondendo a uma orientação para os vetores normais a $N$.

Indicaremos por $\Sigma^{+}$o subconjunto de $\Sigma H$ correspondente aos vetores normais a $N$, que apontam para fora.

Definição 2.1.1 $O$ dual de $N$ é o conjunto dos valores críticos de $H$, isto é, o conjunto $H\left(\Sigma^{+}\right)$.

$A$ aplicação de Gauss está associada a $H$ pela composição

$$
G: N \stackrel{\pi_{1}^{-1}}{\longrightarrow} \Sigma^{+} \rightarrow \mathbb{R} \times S \stackrel{\pi}{\rightarrow} S
$$


Como foi definida, a familia de funções $H$ depende da posição de $N$ com respeito à origem. Contudo é fácil verificar que qualquer translação de $N$ dá uma familia equivalente de funções no seguinte sentido:

Duas familias suaves de funções $F_{i}: N \times S \rightarrow \mathbb{R}, i=1,2$ são equivalentes se existirem difeomorfismos $h_{1}, h_{2}, h_{3}$ comutando o seguinte diagrama:

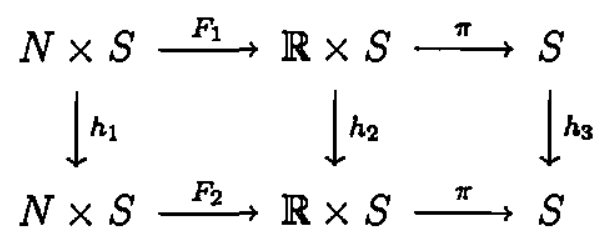

onde $F_{i}(x, y)=\left(f_{i}(x, y), y\right)$ e $\pi$ é a projeção canônica. Observe que a comutatividade do diagrama mostra que a segunda componente de $h_{2}$ depende só de $x$.

$$
h_{2}(t, x)=\left(\left(h_{2}\right)_{1},\left(h_{2}\right)_{2}\right)(t, x)=\left(\left(h_{2}\right)_{1}(t, x), h_{3}(x)\right)
$$

já que $\pi \circ h_{2}(t, x)=h_{3} \circ \pi(t, x)=h_{3}(x)$. Segue assim que existe um difeomorfismo de $\mathbb{R} \times S$ levando o dual de $N$ no dual transladado de $N$. A aplicação de Gauss, fica inalterada.

$O$ relacionamento entre as singularidades da familia $H_{f}$ e as singularidades da aplicação normal de Gauss fica evidente no seguinte lema, provavelmente devido a Milnor:

Lema 2.1.1 $O$ ponto $y \in N$ é um ponto regular de $G: N \rightarrow S$ com $G(y)=u$ se $e$ somente se a função altura $H_{u}: N \rightarrow \mathbb{R}$ tem uma singularidade $A_{1}$ em $y$.

Demonstração: É suficiente considerar o caso $u=(0, \ldots, 1)$, isto é, $H_{u}$ é a função altura $x_{n+1}$. Claramente podemos escrever $N$ localmente como $x_{n+1}=h\left(x_{1}, \ldots, x_{n}\right)$ para algum $h=H_{u}$. Entâo

$$
G\left(x_{1}, \ldots, x_{n}, h\left(x_{1}, \ldots, x_{n}\right)\right)=p\left(-\frac{\partial h}{\partial x_{1}}, \ldots,-\frac{\partial h}{\partial x_{n}}, 1\right)
$$


onde $p: \mathbb{R}^{n+1}-\{0\} \rightarrow S$ é tal que $p(x)=x \cdot\|x\|^{-1}$, onde $\|\cdot\|$ é a norma Euclidiana. Quando restringimos à qualquer hiperplano não passando pela origem $p$ atua como um difeomorfismo local. Então u é um valor regular de $G$ se e somente se $\left(-\frac{\partial^{2} h}{\partial x_{i} x_{j}}\right)$ é não singular. Então $h$ tem singularidade $A_{1}$ se o rank da hessiana é máximo $\Leftrightarrow a$ jacobiana de Gauss é máxima.

Podemos agora formular varias questões. Por exemplo, que tipo de funções altura $H_{u}$ se deve esperar; o que o dual de $N$ parece genericamente? Quais as correspondentes singularidades da aplicação de Gauss?

Com relação à primeira questão, a resposta é dada pelo teorema de genericidade de Looijenga.

Definição 2.1.2 Se $\chi=\cup X_{j}$ é um subconjunto estratificado do espaço de multijatos ${ }_{r} J^{k}(N, \mathbb{R})$, dizemos que $f$ é tangente transversa à $\chi$ se o jato ${ }_{r} j_{1}^{k} H_{f}: N^{(r)} \times S \rightarrow$ ${ }_{r} J^{k}(N, \mathbb{R})$ é transversal à cada $X_{j} \subset \chi$, onde $N^{(r)}=\left\{\left(x_{1}, \ldots, x_{r}\right) \in N^{r}\right.$ tal que $x_{i} \neq x_{j}$ para $\left.i \neq j\right\}$.

Teorema 2.1.1 (Looijenga [19]) Dado $\chi=\cup X_{j}$ como acima, o conjunto de mergulhos $f \in C^{\infty}\left(N, \mathbb{R}^{n+1}\right)$ com $H_{f}$ tangente transversa à $\chi$ é residual.

Os subconjuntos estratificados $\chi=\cup X_{j} \subset{ }_{r} J^{k}(N, \mathbb{R})$ de interesse são subconjuntos em ${ }_{r} J^{k}(N, \mathbb{R})$ invariantes pela ação do grupo $\mathcal{A}^{(k)}=\mathcal{R}^{(k)} \times \mathcal{L}^{(k)}$ dos $k$-jatos de difeomorfismos na fonte e na meta. A aplicação do teorema é feita da seguinte forma: Obtemos primeiramente uma classificação de todas as singularidades em $J^{k}(n, 1)$ de $\mathcal{A}_{e}$-codimensão $\leq n=\operatorname{dim}(S)$ (dimensão do espaço de parâmetros).

Suponhamos que obtivemos um número finito de órbitas nesta classificação. Estratificamos, então, o espaço de jatos (e também o de multijatos) ${ }_{r} J^{k}(N, \mathbb{R}$ ) obtendo $X_{1}, X_{2}, \ldots, X_{s}$, e também o complementar destas órbitas. Este complementar 
pode ser decomposto em um número finito de variedades de codimensão maior que $2 n(\operatorname{dim}(N)+\operatorname{dim}(S)), X_{s+1}, X_{s+2}, \ldots, X_{t}$. Aplicando então o teorema 2.1.1, a esta estratificação, obtemos para cada $X_{j}$, o conjunto

$$
R_{X_{j}}=\left\{f \in C^{\infty}\left(N, \mathbb{R}^{n+1}\right), \text { f mergulho, }{ }_{r} j_{1}^{k} H_{f} \text { é transversal a } X_{j}\right\} \text { é residual }
$$

Logo o conjunto $R=\cap R_{X_{i}}$ é ainda residual.

Observe que para um mergulho $f \in R$, a aplicação $k$-jato associada a $f$ evita os estratos $X_{i}$ para $i>s$ e é transversal $a X_{i}$, para $i \leq s$. Uma tal imersão é chamada uma imersão genérica para a situação que se está considerando. Assim um mergulho genérico $f: N \rightarrow \mathbb{R}$ dá origem a uma composta, $H_{f}: N \times S \rightarrow \mathbb{R}$, a qual apresentará somente aquelas singularidades que aparecem na classificação cuja $\mathcal{A}_{e}$-codimensão seja no máximo $n=\operatorname{dim} S$. A transversalidade às órbitas nos assegura, então, que estas singularidades serão versalmente deformadas (ou desdobradas), ou seja, que a familia $H_{f}$ é localmente versal.

Pode acontecer que, feita a classificação, tenhamos chegado a um número infinito de órbitas, isto é, tenhamos encontrado algum germe modal. Neste caso, devemos reunir estas órbitas em estratos e considerar então, não a codimensão individual das órbitas, mas sim a codimensão do estrato associado. Também não poderemos mais esperar que a aplicação $k$-jato seja transversal às órbitas.

Quando $n \leq 5$, as únicas singularidades de codimensão $\leq n$ em $J^{k}(n, 1)$ são as singularidades simples, classificadas por Arnold. Com efeito, cada estrato $X_{j} \dot{e}$ uma $A^{k}$-órbita de codimensão $\leq 5$ se e somente se $X_{j}$ é simples. Mais ainda, se $f$ é simples com número de Milnor $\mu$, o subconjunto $X_{j}$ correspondente em $J^{k}(N, \mathbb{R})$ terá codimensão $n+\mu-1$, de modo que tais singularidades ocorrem quando $\mu-1 \leq n$. Para as informações sobre o dual de $N$, é preciso investigar as singularidades genéricas que ocorrem quando $n \leq 5$, para germes (caso $r=1$ ) e também para multigermes 
(caso $r>1)$

Para uma melhor discussão da geometria do mergulho genérico $f: N \rightarrow \mathbb{R}^{n+1}$, o seguinte teorema, que complementa o resultado obtido no lema 2.1.1, é essencial:

Teorema 2.1.2 (Looijenga [19], Wall [20]) Seja $f: N \rightarrow \mathbb{R}^{n+1}$ um mergulho de $N$ para o qual a familia $H_{f}$ associada é localmente versal. Então, a aplicação normal de Gauss associada a este mergulho $G: N \rightarrow S$, é localmente estável.

Nas próximas seções estudamos a caracterização geométrica para as singularidades da função altura quando $n=1$ e 2 e as correspondentes singularidades do dual de $N$ $e$ da aplicação normal de Gauss.

\subsection{Curvas planas}

Seja $C$ uma curva regular em $\mathbb{R}^{2}$. O contato de $C$ com retas em $\mathbb{R}^{2}$ é dado pela familia

$$
\begin{aligned}
& H: C \times S^{1} \rightarrow \mathbb{R} \\
& (p, u) \mapsto<p, u>
\end{aligned}
$$

Para cada $u \in S^{1}$ fixo, $H_{u}$ descreve o contato de $C$ com a reta que passa por $p$ e é ortogonal a u. Como consequência do teorema de Looijenga nesta dimensão, segue-se que para um mergulho genérico de $C$ em $\mathbb{R}^{2}$, as singularidades da função $H$ são as singularidades de germes e multigermes de codimensão $\leq 1$. Assim, podem ocorrer as seguintes singularidades $A_{1}$ (Morse), $A_{2}$ (dobra) e também as singularidades $A_{1} A_{1}$ que correspondem a dois pontos críticos não degenerados de mesma altura.

Para interpretar geometricamente estes resultados, tomemos coordenadas de forma que numa vizinhança da origem a curva seja dada pelo gráfico e projetando o mesmo 
na direção do vetor normal $(0,1)$ obtemos: $t \rightarrow(t, f(t)) \stackrel{\pi}{\rightarrow} f(t)$, onde $f(0)=0=$ $f^{\prime}(0)$. Com as hipóteses anteriores teremos que a singularidade será estável $\left(A_{1}\right)$ $\Leftrightarrow f^{\prime \prime}(x) \neq 0$.

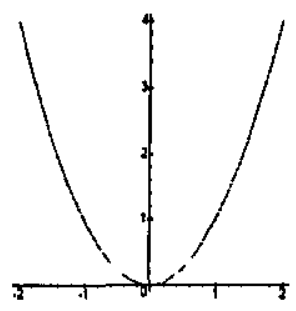

E a singularidade será $A_{2} \Leftrightarrow f^{\prime \prime}(0)=0, f^{\prime \prime \prime}(0) \neq 0$

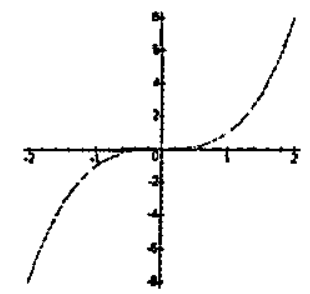

Vamos analisar o dual para os pontos parabólicos (ou de inflexão): Seja $g: \mathbb{R} \rightarrow \mathbb{R}$ dada por $g(x)=x^{3}$, e $H(x, u)=\left(u x-x^{3}, u\right) . O$ dual $H\left(\Sigma^{+}\right)$é portanto $\left\{\left(2 x^{3}, 3 x^{2}\right)\right\}$

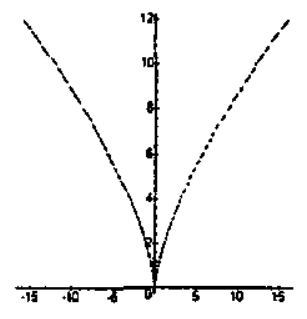

Considerando os multijatos, as singularidades genéricas que podem ocorrer são do tipo $A_{1} A_{1}$ e o dual correspondente possui ponto duplo como singularidade. De 
fato, escolhendo pontos críticos de Morse com a mesma altura: $f_{1}(t)=t^{2}, f_{2}(t)=t^{2}$. Os possiveis desdobramentos versais são: $\left(t^{2}+u, t^{2}\right)$ ou $\left(t^{2}, t^{2}+u\right)$. Considerando $H(t, u)=\left[\left(t^{2}+u, u\right) ;\left(t^{2}, u\right)\right]$ obtemos o dual $H\left(\Sigma^{+}\right)=\{[(u, u) ;(0, u)]\}$

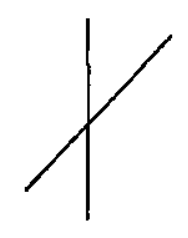

\subsection{Superfícies em $\mathbb{R}^{3}$}

Seja $M$ uma superfície suave em $\mathbb{R}^{3}$. O contato de $M$ com planos em $\mathbb{R}^{3}$ é dado pela famitia de funções altura

$$
\begin{aligned}
H: M \times S^{2} & \rightarrow \mathbb{R} \\
(p, u) & \mapsto<p, u>
\end{aligned}
$$

Para u fixo, a função $H_{u}$ é a função altura na direção u. Em $p \in M$ esta função descreve o contato de $M$ com o plano pelo ponto $p$ cujo vetor normal é $u$.

Escolhamos coordenadas locais em torno de $p_{0}$, tais que $p_{0}=(0,0)$ e a superficie $M$ é dada como o gráfico da função $z=f(x, y)$. Suponhamos que as direções em $S^{2}$ são em torno de $u=(0,0,1)$. Estas direções podem ser parametrizadas por $(a, b, 1)$. Então a família (alterada) de funções altura é dada, localmente, por

$$
\begin{aligned}
H: \mathbb{R}^{2} \times \mathbb{R}^{2}, 0 & \rightarrow \mathbb{R} \\
((x, y),(a, b)) & \mapsto f(x, y)+a x+b y
\end{aligned}
$$

com $H_{0}(x, y)=f(x, y)$. Queremos identificar geometricamente os tipos de singularidades da função $f$ e verificar se a familia $H$ é um desdobramento versal destas 
singularidades. Como $H$ é uma deformação a 2 parâmetros de $f$, somente as singularidades de codimensão $\leq 2$ de $f$ podem ser desdobradas versalmente por $H$, ou seja, somente as singularidades $A_{1}, A_{2}$ e $A_{3}$.

As singularidades estáveis são do tipo $A_{1}: A_{1}(1)$ (sela), $A_{1}(2)$ (Max/Min). $O$ conjunto dos pontos para os quais o plano tangente encontra a superfície em uma singularidade $A_{1}(1)$ são os pontos hiperbólicos e o conjunto dos pontos para os quais o plano tangente encontra a superfície em uma singularidade $A_{1}(2)$ são os pontos elípticos. De fato, $f$ é singular na origem se, e somente se, $f(0,0)=f_{x}(0,0)=$ $f_{y}(0,0)=0$, ou seja, se, e somente, se a direção $(0,0,1)$ é a direção normal a $M$ em $p_{0}$. Fazendo a expansão de Taylor de $f$ temos:

$$
f(x, y)=a x^{2}+2 b x y+c y^{2}+R(x, y), \text { onde } \operatorname{ordem}(R(x, y)) \geq 3
$$

Com uma rotação de forma a colocar os eixos nas direções principais obtemos:

$$
f(x, y)=\frac{k_{1}}{2} x^{2}+\frac{k_{2}}{2} y^{2}+R(x, y)
$$

onde $k_{1}$ e $k_{2}$ são as curvaturas principais em $(0,0)$. Assim, se o ponto é hiperbólico, $k_{1}$ e $k_{2}$ possuem sinais opostos. Logo numa vizinhança da origem, $f(x, y)$ se comporta como uma "sela". Portanto uma pequena translação do plano tangente na direção da normal faz com que este encontre a superfície localmente em uma hipérbole. Se o ponto é elíptico, então $k_{1}$ e $k_{2}$ possuem o mesmo sinal e então $(0,0)$ é um ponto de mínimo ou máximo não degenerado da função altura. Neste caso, nossa singularidade é do tipo $A_{1}(2)$. Ambos os casos descrevem a geometria das singularidades estáveis, isto é, singularidade de Morse numa vizinhança da origem.

Existem três tipos de singularidades genéricas em um ponto parabólico, isto é, ponto para o qual $\kappa=0$. Supondo $k_{2}=0, M(x, y)$ será dado por

$$
M(x, y)=\left(x, y, \frac{k_{1}}{2} x^{2}+R(x, y)\right)
$$


Fazendo mudanças de variáveis, podemos supor $f(x, y)=x^{2}+c x y^{2}+d y^{3}+R(x, y)$, onde a $\operatorname{ordem}(R) \geq 3$.

Se $d \neq 0, f(x, y)=x^{2}+y^{3}+\tilde{R}(x, y)$. Como $x^{2}+y^{3}$ é 3-determinado, podemos desprezar $\tilde{R}(x, y)$ na classificação da singularidade de $f$, que neste caso é do tipo $A_{2}$. Observemos que o plano tangente encontra $N$ em uma curva com cúspide. Podese verificar facilmente que a familia $H(x, y, a, b)=x^{2}+y^{3}+a x+b y$ é versal, isto é , $T \mathcal{A}_{e}(f)+\mathbb{R}\left\{\frac{\partial H}{\partial a}, \frac{\partial H}{\partial b}\right\}=\theta_{f}$. Com cálculos simples utilizando a forma normal, podemos verificar que a condição $d \neq 0$ significa geometricamente que a única direção assintótica no ponto parabólico é transversal a este conjunto.

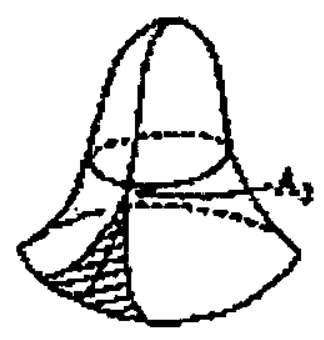

Se $d=0$, tomamos $f(x, y)=x^{2}+x y^{2} \pm y^{4}$, que é uma singularidade de tipo $A_{3}$. Observe que $x^{2}+x y^{2} \pm y^{4}$ é $\mathcal{R}$-equivalente a $x^{2} \pm y^{4}$ e sua $\mathcal{A}_{e}$-codimensão é igual a 2. Além disso, a familia $H(x, y)=x^{2}+x y^{2} \pm y^{4}+a x+b y$ é um desdobramento versal de $f(x, y)=x^{2}+x y^{2} \pm y^{4}$. Com efeito, temos:

$$
<2 x+y^{2}, 2 x y \pm 4 y^{3}>+\mathbb{R}\{x, y\}=<x, y>
$$

Vamos calcular o dual destas singularidades.

Para a familia $H(x, y)=\left(a, b, x^{2}+y^{3}+a x+b y\right)$, temos que o conjunto $\Sigma(H)=$ $\left\{(x, y, a, b) ;(x, y)\right.$ é ponto crítico de $\left.H_{a, b}: \mathbb{R}^{2}, 0 \rightarrow \mathbb{R}, 0\right\}$ é definido pelas equações

$$
\begin{aligned}
& 2 x+a=0 \\
& 3 y^{2}+b=0
\end{aligned}
$$


Portanto $H(\Sigma)$ pode ser descrito pela parametrização

$$
(x, y) \mapsto\left(-2 x,-3 y^{2},-x^{2}-2 y^{3}\right)
$$

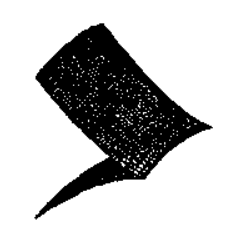

$A$ aplicação de Gauss em uma vizinhança de $(0,0)$ é dada por

$$
\begin{aligned}
& G: \mathbb{R}^{2}, 0 \rightarrow \mathbb{R}^{2}, 0 \\
& (x, y) \mapsto\left(-2 x,-3 y^{2}\right)
\end{aligned}
$$

Observe que a singularidade de $G$ em $(0,0)$ é uma dobra.

Para a familia $H(x, y)=\left(a, b, x^{2}+x y^{2} \pm y^{4}+a x+b y\right)$, o conjunto $\Sigma(H)$ é definido pelas equações:

$$
\begin{gathered}
2 x+y^{2}+a=0 \\
2 x y \pm 4 y^{3}+b=0 \\
\text { Portanto } H(\Sigma)=\left(-2 x-y^{2},-2 x y \mp 4 y^{3},-x^{2}-2 x y^{2} \mp 3 y^{4}\right)
\end{gathered}
$$

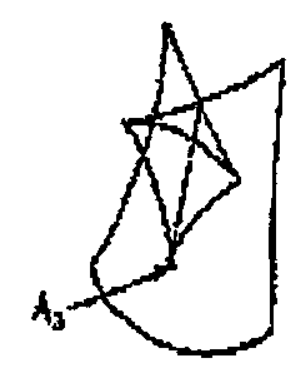


E a aplicação de Gauss $G$ numa vizinhança de $(0,0)$ é dada por

$$
\begin{aligned}
& \mathbb{R}^{2}, 0 \rightarrow \mathbb{R}^{2}, 0 \\
& (x, y) \mapsto\left(-2 x-y^{2},-2 x y \mp 4 y^{3}\right)
\end{aligned}
$$

Note que $(0,0)$ é um ponto de cúspide.

Nas proposições seguintes resumimos os resultados discutidos acima:

Teorema 2.3.1 Seja $f$ um mergulho genérico de $N$ em $\mathbb{R}^{n+1}$. Seja $p$ um ponto singular de $H_{u}: N \rightarrow \mathbb{R}$. Então:

(a) u é direção normal a $N$ em $p$

(b) (i) $p$ é de tipo $A_{1} \Longleftrightarrow K(p) \neq 0$

(ii) $p$ é de tipo $A_{2} \Longleftrightarrow p$ é um ponto parabólico; o conjunto dos pontos parabólicos $\Pi$ é uma curva regular e a única direção assintótica em $p$ é transversal ao conjunto dos pontos parabólicos $\Pi$.

(iii) $p$ é de tipo $A_{3} \Longleftrightarrow p$ é ponto parabólico; o conjunto dos pontos parabólicos $\Pi$ é uma curva regular e a única direção assintótica em p é tangente a $\Pi$.

Proposição 2.3.1 Nas hipóteses acima, seja u um vetor normal a $N$ em $p$.

(i) $p$ é de tipo $A_{1}$ para $H_{u} \Longleftrightarrow p$ é ponto regular para a aplicação normal de Gauss.

(ii) $p$ é de tipo $A_{2}$ para $H_{u} \Longleftrightarrow p$ é ponto de dobra para $G$

(iii) $p$ é de tipo $A_{3}$ para $H_{u} \Longleftrightarrow p$ é um ponto de cúspide para a aplicação de Gauss.

Proposição 2.3.2 (a) O dual de $M$ é localmente difeomorfo a

- uma superfície suave nos pontos $A_{1}$ 
- um edge cuspidal nos pontos $A_{2}$

- um rabo de andorinha num ponto $A_{3}$

(b) $A$ imagem do conjunto parabólico na esfera de Gauss é localmente difeomorfa a

- uma curva suave nos pontos $A_{2}$

- uma cúspide em pontos de tipo $A_{3}$

Uma análise análoga dos multigermes de $\mathcal{A}_{e}$-codimensão $\leq 2$ mostra que:

Proposição 2.3.3 As singularidades genéricas são dois pontos singulares com a mesma imagem:

(i) $A_{1}(1) A_{1}(1)$; (ii) $A_{1}(1) A_{1}(2)$; (iii) $A_{1}$ (2) $A_{1}(2)$; (iv) $A_{1}(1) A_{2}$; (v) $A_{1}(2) A_{2}$;

ou três pontos singulares com a mesma imagem:

(vi) $A_{1}(1) A_{1}(1) A_{1}(1)$; (vii) $A_{1}(1) A_{1}(1) A_{1}(2)$; (viii) $A_{1}(1) A_{1}(2) A_{1}(2)$; (ix) $A_{1}(2) A_{1}(2) A_{1}(2)$.

Os correspondentes duais são cúspide $\times$ reta; ponto duplo $\times$ reta; pontos triplos $e$ rabo de andorinha (correspondente as singularidades do tipo $A_{3}(1)$ e $A_{3}(2)$ ). 


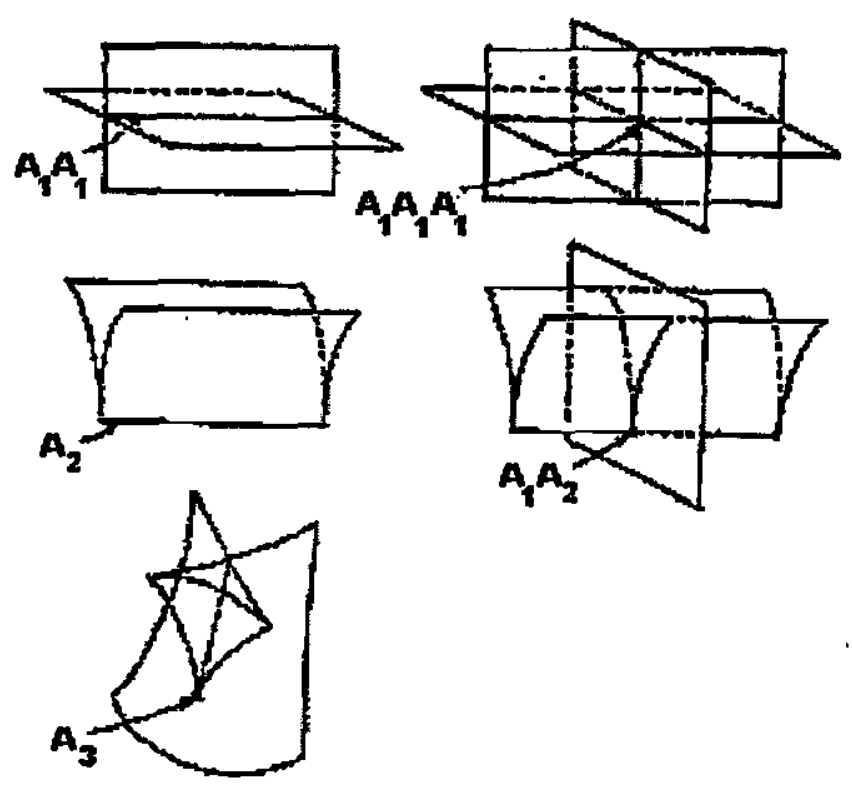

Observação 2.3.1 O livro "Cusps of the Gauss mappings"de T. Banchoff, T. Gaffney e C. McCrory [21], descreve várias caracterizações geométricas para as cúspides da aplicação de Gauss.

Destacamos a seguir uma destas caracterizações que será útil na parte final do trabalho:

Teorema: Para uma superfície regular genérica, as cúspides da aplicação de Gauss são precisamente os pontos parabólicos os quais são os limites dos pontos de inflexão das curvas assintóticas. 


\section{Capítulo 3}

\section{Duais de hipersuperfícies projetivas}

Neste capítulo, demonstraremos teoremas de genericidade para os contatos de hipersuperfícies algébricas reais e complexas com familias de hiperplanos de $\mathbb{P}^{n+1}$. A principal referência é o artigo "The Duals of Generic Hypersurfaces"de J.W. Bruce [13].

Sejam $K$ o corpo dos números reais ou complexos, $\mathbb{P}^{n+1}$ o espaço projetivo real ou complexo de dimensão $n+1$, e $K^{M}$ o espaço vetorial dos polinômios homogêneos de grau d em $n+2$ variáveis $\left(M=\left(\begin{array}{c}n+d+1 \\ d\end{array}\right)\right)$ e $\{F=0\}$ uma hipersuperfície não singular em $\mathbb{P}^{n+1}$.

Queremos discutir a estrutura dos duais de hipersuperfícies projetivas "genéricas" $\{F=$ $0\}$, e como estamos trabalhando no espaço projetivo precisamos tomar cuidado pois as funções altura são objetos Euclidianos. Contudo, transversalidade é um fenômeno local, de modo que podemos dar uma definição local de transversalidade tangente. Para fazer isto, selecionaremos uma carta afim de $\mathbb{P}^{n+1}$ e é claro que nossa definição 
de transversalidade tangente deve ser independente da carta e de qualquer outra escolha desejada. Como no capitulo anterior, consideraremos apenas subvariedades de singularidades naturais do espaço de jatos e multijatos resultantes das $\mathcal{R}^{(k)} \times \mathcal{L}^{(k)}$ subvariedades invariantes $X_{j} \subset \Sigma \subset{ }_{r} J^{k}(n, 1)$, e a definição de transversalidade tangente assegurará que para uma hipersuperfície não singular $V=\{F=0\}$ transversa tangente $a X_{j}$, o conjunto $\left\{x \in V ;\right.$ a função altura normal à $x$ é do tipo $\left.X_{j}\right\}$ é uma subvariedade de $V$ da dimensão correta.

Os principais resultados discutidos em [13] e apresentados neste capitulo são os teoremas abaixo, que são respectivamente, os teoremas de genericidade para germes $e$ multigermes.

Teorema 3.2.1: Seja $D \subset K^{M}$ o subconjunto das formas que definem hipersuperfícies singulares. Cada $F \in K^{M}-D$ possui uma vizinhança $U \subset K^{M}-D$ e no caso $K=\mathbb{C}$ (respectivamente $K=\mathbb{R}$ ) um subconjunto subanalítico (respectivamente real subanalítico) $B \subset U$ de codimensão real $\geq 1$, tal que para todo $G \in U-B a$ hipersuperfície $\{G=0\}$ é tangente transversa à $X \subset J^{k}(n, 1)$ desde que $k \leq d$.

Teorema 3.3.2: Seja $K=\mathbb{C}$. Seja $\{F=0\}$ uma hipersuperfície não singular com tangências em posição geral. Então dada uma uma estratificação natural $\chi \subset$ $\Sigma \subset J^{k}(n, 1)$, existe uma vizinhança aberta $U$ de $F$ e uma familia enumerável de subconjuntos reais subanalíticos $B_{\alpha} \subset U, \operatorname{com} \operatorname{cod}\left(B_{\alpha}\right) \geq 1$ tal que para $G \in U-\cup B_{\alpha}$ a hipersuperfície $\{G=0\}$ é tangente multitransversa a $\chi$ desde que $k \leq \frac{d-1}{2}$.

Nosso objetivo é apresentar as provas detalhadas destes teoremas e aplicar os resultados ao estudo dos duais de hipersuperfícies projetivas. 


\subsection{A família de funções altura: definições básicas e resultados preliminares}

Seja $D \subset K^{M}$ o subconjunto algébrico das formas que definem hipersuperfícies singulares, ou seja, não possuem rank máximo, e seja $F \in K^{M}-D$.

Se $\Gamma_{F}=\left\{(x, L) \in \mathbb{P}^{n+1} \times \mathbb{P}^{n+1} ; x \in L \cap\{F=0\}\right\} e \Pi_{F}: \Gamma_{F} \rightarrow \mathbb{P}^{n+1}$ é a projeção canônica, então o conjunto singular de $\Pi_{F}, \Sigma \Pi_{F}=\{(x, L) ; L$ é o plano tangente à $\{F=0\}$ em $x\}$.

Definição 3.1.1 $O$ dual de $\{F=0\}$ é o conjunto dos valores críticos de $\Pi_{F}$, isto é $\Pi_{F}\left(\Sigma \Pi_{F}\right)$.

Proposição 3.1.1 O dual de $\Pi_{F}$ é um conjunto algébrico.

Demonstração: A projeção $\Pi_{F}: \mathbb{P}^{n+1} \times \mathbb{P}^{n+1} \rightarrow \mathbb{P}^{n+1}$ é fechada na topologia de Zariski quando $K=\mathbb{C}$ (ver [11]), logo, leva conjuntos fechados em conjuntos fechados e neste caso os duais são algébricos.

Seja $x \in V$ e escolha uma carta afim $K^{n+1} \subset \mathbb{P}^{n+1}$ contendo $x$, seja $V_{1}=$ $V \cap K^{n+1}$. Sem perda de generalidade, podemos supor que $x=0 \in K^{n+1}$.

$A$ variedade irredutivel afim $V_{1}$ será o conjunto de zeros de algum polinômio irredutivel $f$ e podemos escolher uma parametrização local de $V_{1}$ no ponto $0, h: K^{n} \rightarrow V_{1}$ (de modo que $f \circ h(\alpha) \equiv 0)$.

Se $\left(K^{n+1}\right)^{*}$ é o dual de $K^{n+1}$, seja df $(0) \in\left(K^{n+1}\right)^{*}$ a diferencial de $f$ em 0.

Escolheremos então uma aplicação linear $L: K^{n} \rightarrow\left(K^{n+1}\right)^{*}$ de modo que

$$
\operatorname{ImL} \oplus S p\{d f(0)\}=\left(K^{n+1}\right)^{*}
$$


Observe que ImL é um subespaço vetorial de $\left(K^{n+1}\right)^{*}$ de dimensão $n$

Definimos então um germe de uma famitia a n parâmetros de funções $H_{a}: K^{n} \rightarrow$ $K$ por $H(f, h, L):\left(K^{n} \times K^{n}, 0\right) \rightarrow(K, 0)$ onde $H(f, h, L)(\alpha, a)=(d f(0)+L(a))(h(\alpha))$

Lema 3.1.1 (1) $H$ é independente das escolhas da equação $f$, da parametrização $h$ $e$ da aplicação linear $L$, isto é, quaisquer duas escolhas dão familias equivalentes.

(2) Seja $\Sigma H=\left\{(\alpha, a) ; \alpha\right.$ é um ponto crítico de $\left.H_{a}\right\}$. Então o conjunto dos pontos críticos de $H$ é regular e a projeção $\Sigma H \rightarrow K^{n},(\alpha, a) \rightarrow \alpha$ é um difeomorfismo local.

Demonstração: (1)(a) Como $V_{1}$ é uma variedade afim irredutivel, qualquer outra equação definindo $V_{1}$ é da forma $\lambda f=0$, para algum $\lambda \in K-\{0\}$. Usando as mudanças de coordenadas $\psi(\alpha, a)=(\alpha, \lambda a)$ e $\phi(c)=\lambda c$, temos que:

$$
\phi \circ H(f, h, L)(\alpha, a)=\phi((d f(0)+L(a))(h(\alpha)))=\lambda(d f(0)+L(a))(h(\alpha))
$$

Por outro lado, temos:

$$
H(\lambda f, h, L) \circ \psi(\alpha, a)=H(\lambda f, h, L)(\alpha, \lambda a)=\lambda(d f(0)+L(a))(h(\alpha))
$$

Portanto, $\phi \circ H(f, h, L)(\alpha, a)=H(\lambda f, h, L) \circ \psi(\alpha, a)$.

(b) Quaisquer duas parametrizações $h_{1}, h_{2}$ de $V_{1}$ no ponto 0 diferem por um difeomorfismo $h:\left(K^{n}, 0\right) \rightarrow\left(K^{n}, 0\right)$ tal que $h_{2}=h_{1} \circ h$. Uma mudança de coordenadas $\psi(\alpha, a)=(h(\alpha), a)$ nos fornece:

$$
\begin{aligned}
& \left(H\left(f, h_{1}, L\right) \circ \psi\right)(\alpha, a)=H\left(f, h_{1}, L\right)(h(\alpha), a)=(d f(0)+L(a))\left(h_{1}(h(\alpha))=\right. \\
& =(d f(0)+L(a))\left(h_{2}(\alpha)\right)=H\left(f, h_{2}, L\right)(\alpha, a)
\end{aligned}
$$

(c) Suponha que temos duas aplicações lineares $L_{1}, L_{2}$ como acima. Podemos escrever $L_{1}(a)=\theta(a) d f(0)+\varphi(a)$ para alguma aplicação linear $\theta: K^{n} \rightarrow K$ e $\varphi: K^{n} \rightarrow$ ImL $L_{2}$. Note que $\varphi$ é um isomorfismo. De fato, suponha que $\varphi$ não seja, então 
existe $a \neq 0, a \in K^{n}$ tal que $\varphi(a)=0$. Como $L_{1}(a) \neq 0$ ( $L_{1}$ é isomorfismo) $e$ $\theta(a) \neq 0$, segue que $L_{1}(a)=\theta(a) d f(0)$, o que é absurdo devido à hipótese de que $\operatorname{Im} L_{2} \oplus \operatorname{Sp}\{d f(0)\}=\left(K^{n+1}\right)^{*}$.

Através das seguintes mudanças de coordenadas: $\psi(\alpha, a)=\left(\alpha, L_{2}{ }^{-1}\left(\frac{\varphi(a)}{1+\theta(a)}\right)\right)$, $\phi(c, a)=\left(\frac{c}{1+\theta(a)}\right)$ e usando o fato de que $L_{1}(a)=\theta(a) d f(0)+\varphi(a)$, obtemos:

$$
\begin{aligned}
& \phi\left(H\left(f, h, L_{1}\right)(\alpha, a), a\right)=\phi\left(\left(d f(0)+L_{1}(a)\right)(h(\alpha)), a\right)= \\
& \phi((d f(0)+\theta(a) d f(0)+\varphi(a))(h(\alpha)), a)=\phi((1+\theta(a)) d f(0)+\varphi(a))(h(\alpha)), a)= \\
& \left(d f(0)+(1+\theta(a))^{-1} \varphi(a)\right)(h(\alpha))
\end{aligned}
$$

Por outro lado, temos

$$
\begin{aligned}
& \left(H\left(f, h, L_{2}\right) \circ \psi\right)(\alpha, a)=H\left(f, h, L_{2}\right)\left(\alpha, L_{2}^{-1}\left(\frac{\varphi(a)}{1+\theta(a)}\right)\right)= \\
& =\left(d f(0)+L_{2}\left(L_{2}^{-1}\left(\frac{\varphi(a)}{1+\theta(a)}\right)\right)(h(\alpha))=\right. \\
& =\left(d f(0)+(1+\theta(a))^{-1} \varphi(a)\right)(h(\alpha))
\end{aligned}
$$

Portanto,

$$
\phi\left(H\left(f, h, L_{1}\right)(\alpha, a), a\right)=\left(H\left(f, h, L_{2}\right) \circ \psi\right)(\alpha, a)
$$

(2) Temos $H(f, h, L)(\alpha, a)=(d f(0)+L(a))(h(\alpha))$. Assim, $T_{\alpha} H(f, h, L)=T_{\alpha}(d f(0)+$ $L(a)) \circ T_{\alpha} h=(d f(0)+L(a)) \circ T_{\alpha} h$. Como $h: K^{n} \rightarrow K^{n+1}$, temos $T_{\alpha} h: T_{\alpha} K^{n} \rightarrow$ $T_{h(\alpha)} K^{n+1}$, mas $T_{\alpha} K^{n} \approx K^{n}$ e $T_{h(\alpha)} K^{n+1} \approx K^{n+1}$, assim obtemos o seguinte diagra$m a:$

$$
K^{n} \stackrel{T_{\alpha} h}{\longrightarrow} K^{n+1} \stackrel{d f(0)+L(a)}{\longrightarrow} K
$$

Vamos definir a aplicação induzida de $T_{\alpha} h$ da seguinte forma:

$$
\begin{aligned}
\left(T_{\alpha} h\right)^{*}:\left(K^{n+1}\right)^{*} & \rightarrow\left(K^{n}\right)^{*} \\
A & \mapsto\left(T_{\alpha} h\right)^{*}(A)=A \circ T_{\alpha} h
\end{aligned}
$$

Assim, o conjunto dos pontos críticos de $H(f, h, L)$ fica definido pelo conjunto

$$
\Sigma=\left\{(\alpha, a) ;\left(T_{\alpha} h\right)^{*}(d f(0)+L(a))=0\right\}
$$


Escrevendo $\xi(\alpha, a)=\left(T_{\alpha} h\right)^{*}(d f(0)+L(a))$, vemos que $\frac{\partial \xi}{\partial a}(0,0)=\left(T_{0} h\right)^{*} L$ : $K^{n} \rightarrow\left(K^{n}\right)^{*}$, que é invertivel, então pelo Teorema da Função Implícita, podemos parametrizar $\Sigma$ como $(\alpha, \beta(\alpha))$ para algum $\beta$, seguindo assim, o resultado.

Vamos deixar de lado os parênteses $(f, h, L)$ e considerar a aplicação

$$
\begin{aligned}
j_{1}^{k} H_{a}:\left(K^{n} \times K^{n},(0,0)\right) & \rightarrow\left(J^{k}\left(K^{n}, K\right), j_{1}^{k} H(0,0)\right) \\
(\alpha, a) & \mapsto j^{k} H_{a}(\alpha)
\end{aligned}
$$

Definição 3.1.2 Uma hipersuperfície projetiva $V \subset \mathbb{P}^{n+1}$ é tangente transversa à $\mathcal{R}^{(k)} \times \mathcal{L}^{(k)}$-estratificação invariante $\chi=\cup X_{j} \subset \Sigma \subset J^{k}(n, 1)$ se para cada $x \in V$ pudermos escolher uma carta afim e uma vizinhança $A_{1} \times A_{2}$ de $(0,0) \in K^{n} \times K^{n}$ de modo que a aplicação $j_{1}^{k} H_{a}: A_{1} \times A_{2} \rightarrow J^{k}\left(K^{n}, K\right)$ é transversal à correspondente subvariedade $\tilde{X}_{j} \subset J^{k}\left(K^{n}, K\right)$.

Segue do lema 3.1 .1 acima que se $j_{1}^{k} H_{x}: A_{1} \times A_{2} \rightarrow J^{k}\left(K^{n}, K\right)$ é transversal à $X_{j}$ então para um y suficientemente próximo de $x, j_{1}^{k} H_{y}$ também será transversal a $X_{j}$.

Proposição 3.1.2 Se $X$ é uma subvariedade $\mathcal{R}^{(k)} \times \mathcal{L}^{(k)}$-invariante de $\Sigma \subset J^{k}(n, 1)$ e $V \subset \mathbb{P}^{n+1}$ é tangente transversa $\dot{a} X$, então o conjunto $V(X)=\{x \in V ;$ a função altura normal à $x$ é do tipo $X\}$ é regular e cod $V(X)$ em $V$ é igual à cod $X$ em $\Sigma$. (Observe que esta proposição segue do lema 3.1 .1 e do lema da transversalidade.)

Queremos mostrar que as hipersuperfícies $\{F=0\}$ que não são tangente transversas a algum subconjunto invariante $X \subset \Sigma$ são escassas. Para obter um resultado forte, assumiremos que no caso real $X$ é semi-algébrico e no caso complexo $X$ é construtivel. 
Primeiramente vamos definir o que vem a ser um conjunto semialgébrico e um conjunto construtivel:

Definição 3.1 .3 ([3]) Um conjunto $A \subset \mathbb{R}^{n}$ é semialgébrico quando ele pode ser obtido através da combinação de um número finito das seguintes operações: interseção, união e diferença de conjuntos, a partir de conjuntos da forma $\left\{x \in \mathbb{R}^{n} ; f(x)>\right.$ $0\} \operatorname{com} f: \mathbb{R}^{n} \rightarrow \mathbb{R}$ uma função polinomial.

Definição 3.1.4 ([14]) Um subconjunto $S$ de $\mathbb{C}^{n}$ ou $\mathbb{P}^{n}$ é construtivel se ele pertence a uma álgebra Booleana gerada por conjuntos algébricos fechados, ou equivalentemente, se $S$ é uma união disjunta $T_{1} \cup \ldots \cup T_{k}$, onde $T_{i}$ é localmente fechado, isto é, $T_{i}=T_{i}^{\prime}-T_{i}^{\prime \prime}$, onde $T_{i}^{\prime}$ é um conjunto algébrico fechado e $T_{i}^{\prime \prime} \subset T_{i}^{\prime}$ um conjunto algébrico menor.

Teorema 3.1.1 (Teorema de Chevalley [23]) Seja A um anel Noetheriano e B uma A-álgebra de tipo finito. Seja $\phi: A \rightarrow B$ o isomorfismo canônico; Seja $X=$ $\operatorname{Spec}(A), Y=\operatorname{Spec}(B)$ e $f=\phi^{*}: Y \rightarrow X$, entäo a imagem $f\left(Y^{\prime}\right)$ de um conjunto construtivel $Y^{\prime}$ em $Y$ é construtivel em $X$.

Teorema 3.1.2 ([14]) Seja $S \subset \mathbb{C}^{n} \times \mathbb{C}^{m}$ um conjunto construtível. Então $\pi_{2}(S) \subset$ $\mathbb{C}^{m}$ é um conjunto construtível. Em particular, se $S$ é uma subvariedade de $\mathbb{C}^{n+m} e$ $\overline{\pi_{2}(S)}$ é o fecho de Zariski da imagem, então $\pi_{2}(S)$ contém um conjunto aberto Zariski em $\overline{\pi_{2}(S)}$

As seguintes definições serão utilizadas:

Seja $\mathcal{A}$ um anel de funções de valores reais definidas em um conjunto $E$. Seja $S(\mathcal{A})$ os subconjuntos de $E$ que são descritos por $\mathcal{A}$, isto é, a menor familia de 
subconjuntos de $E$ contendo todos $\{f(x)>0\}, f \in \mathcal{A}$, que é estável com relação à interseção finita, união finita e complemento.

Equivalentemente, $S(\mathcal{A})$ representa os subconjuntos de $E$ da forma $X=\cup_{i=1}^{p} \cap_{j=1}^{q}$ $X_{i j}$, onde cada $X_{i j}$ é $\left\{f_{i j}(x)=0\right\}$ ou $\left\{f_{i j}(x)>0\right\}, f_{i j} \in \mathcal{A}$.

Seja $M$ uma variedade real analítica. Se $U$ é um subconjunto aberto de $M$, seja $\mathcal{O}(U)$ o anel das funções reais analíticas em $U$.

Definição 3.1.5 ([22]) Um conjunto $X$ de $M$ é semianalítico se para todo $a \in M$ existir uma vizinhança $U$ tal que $X \cap U \in S(\mathcal{O}(U))$.

Definição 3.1.6 ([22]) Um subconjunto $X$ de $M$ é subanalítico se cada ponto de $M$ admite uma vizinhança $U$ tal que $X \cap U$ é uma projeção de um conjunto semianalítico relativamente compacto(isto é, existe uma variedade real analítica $N$ e um subconjunto $A \subset M \times N$, onde $A$ é semianalítico relativamente compacto tal que $X \cap U=\pi(A)$, onde $\pi: M \times N \rightarrow M$ é a projeção).

As propriedades básicas destes conjuntos são também discutidas em [22]

\subsection{O teorema de genericidade local}

Teorema 3.2.1 Cada $F \in K^{M}-D$ possui uma vizinhança $U \subset K^{M}-D$ e no caso $K=\mathbb{C}$ (respectivamente $K=\mathbb{R}$ ) um subconjunto subanalítico (respectivamente real subanalítico) $B \subset U$ de codimensão real $\geq 1$, tal que para todo $G \in U-B$ a hipersuperfície $\{G=0\}$ é tangente transversa à $X \subset J^{k}(n, 1)$ desde que $k \leq d$. 
Usando o fato que $\{F=0\} \subset \mathbb{P}^{n+1}$ é compacto, a demonstração do teorema segue da seguinte afirmação:

Afirmação 3.2.1 Para cada $F \in K^{M}-D$, e $x(0) \in\{F=0\}$ podemos encontrar vizinhanças $A$ (respectivamente $U$ ) de $x(0) \in \mathbb{P}^{n+1}$ (respectivamente $F \in K^{M}-D$ ) e um conjunto ruim $B \subset U$ como acima, de modo que para $G \in U-B$ e $y \in A \cap\{G=0\}$ o germe de aplicação $j_{1}^{k} H_{y}($ para $\{G=0\})$ é transversal à $\tilde{X}$.

Para mostrarmos esta afirmação, usaremos o lema 1.4.1 de Thom. Mas neste caso, as familias de deformações serão obtidas variando as hipersuperfícies $\{G=0\}$.

Demonstração da afirmação 3.2.1: Sem perda de generalidade, vamos supor que $x(0)=$ $(1,0, \ldots, 0)$, vamos também supor que o plano tangente a $F=0$ em $x(0)$ é $x_{1}=0$ e por simplicidade, nesta seção, usaremos a notação $x(0)=(1,0)$, sempre que não houver perigo de confusão. Em particular, estamos assumindo que $\frac{\partial F}{\partial x_{j}}(1,0)=0,1<$ $j \leq n$ e $\frac{\partial F}{\partial x_{1}}(1,0) \neq 0$. Usaremos a carta afim usual em $(1,0, \ldots, 0) \in \mathbb{P}^{n+1}$.

Considere a aplicação $g: K^{M} \times K^{n} \rightarrow K, g(u, x)=F(1, x)+\sum_{1}^{M} u_{i} \varphi_{i}(1, x)$, onde $\left\{\varphi_{1}, \ldots, \varphi_{M}\right\}$ é uma base monomial para o espaço vetorial das formas homogêneas em $n+2$ variáveis de grau $d$. Sem perda de generalidade, podemos assumir que $\varphi_{1}=x_{0}^{d}$ $\left(\log o, \varphi_{1}(1,0)=1\right)$.

Desejamos parametrizar estas superfícies (pelo menos numa vizinhança de $0 \in$ $\left.K^{n+1}\right)$ de modo que procuramos $h: K^{M} \times K^{n} \rightarrow K$ tal que $g(u, h(u, \alpha), \alpha) \equiv 0$. Mas $\frac{\partial g}{\partial x_{1}}=\frac{\partial F}{\partial x_{1}}+\sum u_{i} \frac{\partial \varphi_{i}}{\partial x_{1}}, \log o$

$$
\frac{\partial g}{\partial x_{1}}(0,0)=\frac{\partial F}{\partial x_{1}}(1,0) \neq 0
$$

Assim, pelo teorema da função implícita tal função analítica $h$ existe e para valores pequenos de $u$, a hipersuperfície $g(u, x)=0$, é dada por $(h(u, \alpha), \alpha)$.

Note que quando aplicamos o T.F.I., podemos considerar uma família de gráficos na 
qual $h(u, 0)=0$, ou seja, $h\left(u, \alpha_{2}, \ldots, \alpha_{n}\right)=h(0, \alpha)+u \tilde{h}(u, \alpha)$, onde $h(0,0)=0 e$ $\tilde{h}(u, 0)=0$. Segue ainda que $\frac{\partial h}{\partial u_{i}}(u, 0)=0$.

Agora, temos que escolher a aplicaçâa linear $L$ :

$$
L\left(a_{1}, \ldots, a_{n}\right)=L(a)=\sum_{1}^{n} a_{j} x_{j+1}
$$

Vemos que esta é uma escolha admissivel para $g_{u}=0$ próximo a $x(0)$ tomando-se $u$ suficientemente pequeno. Com efeito,

$$
d g_{u}(0)=\left(\frac{\partial F}{\partial x_{1}}(1,0)+\sum_{1}^{M} u_{i} \frac{\partial \varphi_{i}}{\partial x_{1}}(1,0), \ldots, \frac{\partial F}{\partial x_{n}}(1,0)+\sum_{1}^{M} u_{i} \frac{\partial \varphi_{i}}{\partial x_{n}}(1,0)\right)
$$

No nosso caso, $\frac{\partial F}{\partial x_{i}}(1,0)=0,1<i \leq n$

$$
d g_{u}(0)=\left(\frac{\partial F}{\partial x_{1}}(1,0)+\sum_{1}^{M} u_{i} \frac{\partial \varphi_{i}}{\partial x_{1}}(1,0), \ldots, \sum_{1}^{M} u_{i} \frac{\partial \varphi_{i}}{\partial x_{n}}(1,0)\right)
$$

Logo, L satisfaz a condição

$$
\operatorname{ImL} \oplus S p\left\{d g_{u}(0)\right\}=\left(K^{n+1}\right)^{*}
$$

para u próximo a zero.

Assim, temos uma familia de funções $H: K^{M} \times K^{n} \times K^{n} \rightarrow K$,

$$
\begin{aligned}
& H(u, \alpha, a)=\left(d g_{u}(0)+L(a)\right)(h(u, \alpha), \alpha)= \\
& \left(\frac{\partial F}{\partial x_{1}}(1,0)+\sum_{1}^{M} u_{i} \frac{\partial \varphi_{i}}{\partial x_{1}}(1,0), \ldots, \sum_{1}^{M} u_{i} \frac{\partial \varphi_{i}}{\partial x_{n}}(1,0)\right)(h(u, \alpha), \alpha)+ \\
& +\left(\sum_{1}^{n} a_{j} x_{j+1}\right)(h(u, \alpha), \alpha)= \\
& \left(\frac{\partial F}{\partial x_{1}}(1,0)+\sum_{1}^{M} u_{i} \frac{\partial \varphi_{i}}{\partial x_{1}}(1,0)\right)(h(u, \alpha))+\sum_{i=1}^{M} \sum_{j=2}^{n} u_{i} \frac{\partial \varphi_{i}}{\partial x_{j}}(1,0) \alpha_{j}+\sum_{1}^{n} a_{j} x_{j+1} \alpha_{j+1}
\end{aligned}
$$

o que nos leva à uma aplicação

$$
\eta=j^{k} H_{(u, a)}(\alpha): K^{M} \times K^{n} \times K^{n} \rightarrow J^{k}\left(K^{n}, K\right)=K^{n} \times K \times J^{k}(n, 1)
$$


O principal resultado que precisamos mostrar é que $\eta$ é uma submersão em $(0,0,0)$ se $k \leq d$.

Podemos tomar $k=d$. Como $g(u, h(u, \alpha), \alpha) \equiv 0$, derivando com relação $a u_{i}$, obtemos:

$$
\text { (i) } \quad \frac{\partial g}{\partial u_{i}}(u, h(u, \alpha), \alpha)+\frac{\partial g}{\partial x_{1}}(u, h(u, \alpha), \alpha) \frac{\partial h}{\partial u_{i}}(u, \alpha) \equiv 0
$$

Temos que mostrar que a aplicação tangente

$$
T_{0} \eta: K^{M} \times K^{n} \times K^{n} \rightarrow K^{n} \times K \times J^{d}(n, 1)
$$

é sobrejetora. Aqui, estamos identificando um espaço vetorial com seu espaço tangente no ponto. Observe que $T_{0} \eta\left(0 \times K^{n} \times 0\right)$ contém $K^{n} \times 0 \times 0$

Se $u_{1}$ é a coordenada correspondente ao monômio $x_{0}{ }^{d}$ temos:

$$
\begin{aligned}
& \frac{\partial H}{\partial u_{1}}(0,0)=\frac{\partial f}{\partial x_{1}}(0) \frac{\partial h}{\partial u_{1}}(0,0)+\frac{\partial \varphi_{1}}{\partial x_{1}}(1,0) h(0,0)+ \\
& +\sum_{1}^{M} u_{i} \frac{\partial \rho_{i}}{\partial x_{1}}(1,0) \frac{\partial h}{\partial u_{1}}(0,0)+\sum_{j=2}^{n} \frac{\partial \varphi_{1}}{\partial x_{j}}(1,0) \alpha_{j} \\
& \text { Assim, } \frac{\partial H}{\partial u_{1}}(0,0)=\frac{\partial f}{\partial x_{1}}(0) \frac{\partial h}{\partial u_{1}}(0,0)
\end{aligned}
$$

Pelo teorema da função implícita segue que

$$
\begin{aligned}
& \frac{\partial h}{\partial u_{1}}(0,0)=\frac{-\frac{\partial g}{\partial u_{1}}(0,0)}{\frac{\partial g}{\partial x_{1}}(0,0)}, \text { como } \frac{\partial g}{\partial x_{1}}(0,0)=\frac{\partial f}{\partial x_{1}}(0) \\
& \frac{\partial H}{\partial u_{1}}(0,0)=-\frac{\partial g}{\partial u_{1}}(0,0)=-\varphi_{1}(1,0)=-1 \neq 0
\end{aligned}
$$

Assim, é suficiente mostrar que $\mathcal{L}=\left\{u \in K^{M} ; \sum u_{i} \varphi_{i}\right.$ não possui termos envolvendo $x_{1}$ e nem o termo $\left.x_{0}^{d}\right\}$ é levado sobrejetivamente por $T_{0} \eta$ em $J^{d}(n, 1)$. (Note que ambos os espaços $\mathcal{L}$ e $J^{d}(n, 1)$ possuem a mesma dimensão.) 
A seguinte igualdade se verifica:

(ii) $\quad \frac{\partial}{\partial u_{i}}\left(\frac{\partial g}{\partial x_{1}}(u, 0) h(u, \beta)-\frac{\partial g}{\partial x_{1}}(0,0) h(u, 0)\right)=\frac{\partial}{\partial u_{i}}(H(u, \beta, 0)-H(u, 0,0))$

De fato, temos:

$$
\begin{aligned}
& \frac{\partial}{\partial u_{i}}(H(u, \beta, 0)-H(u, 0,0))= \\
& =\left[\frac{\partial F}{\partial x_{1}}(1,0) \frac{\partial h}{\partial u_{i}}(u, \beta)+\frac{\partial \varphi_{i}}{\partial x_{1}}(1,0) h(u, \beta)+\sum_{1}^{M} u_{i} \frac{\partial \varphi_{i}}{\partial x_{1}}(1,0) \frac{\partial h}{\partial u_{i}}(u, \beta)\right]+ \\
& -[\frac{\partial F}{\partial x_{1}}(1,0) \underbrace{\frac{\partial h}{\partial u_{i}}(u, 0)}_{=0}+\frac{\partial \varphi_{i}}{\partial x_{1}}(1,0) \underbrace{h(u, 0)}_{=0}+\sum_{1}^{M} u_{i} \frac{\partial \varphi_{i}}{\partial x_{1}}(1,0) \underbrace{\frac{\partial h}{\partial u_{i}}(u, 0)}_{=0}]
\end{aligned}
$$

Portanto

(a) $\quad \frac{\partial}{\partial u_{i}}(H(u, \beta, 0)-H(u, 0,0))=\left(\frac{\partial F}{\partial x_{1}}(1,0) \frac{\partial h}{\partial u_{i}}(u, \beta)+\sum_{1}^{M} u_{i} \frac{\partial \varphi_{i}}{\partial x_{1}}(1,0) \frac{\partial h}{\partial u_{i}}(u, \beta)\right)+$ $+\frac{\partial \varphi_{i}}{\partial x_{1}}(1,0) h(u, \beta)$

Por outro lado, temos:

$$
\begin{aligned}
& \frac{\partial}{\partial u_{i}}\left(\frac{\partial g}{\partial x_{1}}(u, 0) h(u, \beta)-\frac{\partial g}{\partial x_{1}}(0,0) h(u, 0)\right)= \\
& \underbrace{\frac{\partial^{2} g}{\partial x_{1} \partial u_{i}}(u, 0)}_{=\frac{\partial \varphi_{i}}{\partial x_{1}}(1,0)} h(u, \beta)+\frac{\partial g}{\partial x_{1}}(u, 0) \frac{\partial h}{\partial u_{i}}(u, \beta)+ \\
& -\frac{\partial^{2} g}{\partial x_{1} \partial u_{i}}(0,0) \underbrace{h(u, 0)}_{=0}-\frac{\partial g}{\partial x_{1}}(0,0) \underbrace{\frac{\partial h}{\partial u_{i}}(u, 0)}_{=0}= \\
& =\frac{\partial g}{\partial x_{1}}(u, 0) \frac{\partial h}{\partial u_{i}}(u, \beta)+\frac{\partial \varphi_{i}}{\partial x_{1}}(1,0) h(u, \beta)
\end{aligned}
$$


Como $\frac{\partial g}{\partial x_{1}}(u, 0)=\frac{\partial F}{\partial x_{1}}(1,0)+\sum_{1}^{M} u_{i} \frac{\partial \varphi_{i}}{\partial x_{1}}(1,0)$, segue que $(a)=(b)$ e consequentemente, (ii) está mostrado! Assim,

$$
\begin{aligned}
& \frac{\partial}{\partial u_{i}}(H(u, \beta, 0)-H(u, 0,0))=\left(\frac{\partial F}{\partial x_{1}}(1,0) \frac{\partial h}{\partial u_{i}}(u, \beta)+\sum_{1}^{M} u_{i} \frac{\partial \varphi_{i}}{\partial x_{1}}(1,0) \frac{\partial h}{\partial u_{i}}(u, \beta)\right)+ \\
& \frac{\partial \varphi_{i}}{\partial x_{1}}(1,0) h(u, \beta) \\
& \quad e \quad \frac{\partial}{\partial u_{i}}(H(u, \beta, 0)-H(u, 0,0))=\frac{\partial g}{\partial x_{1}}(u, 0) \frac{\partial h}{\partial u_{i}}(u, \beta)+\frac{\partial \varphi_{i}}{\partial x_{1}}(1,0) h(u, \beta)
\end{aligned}
$$

Lembrando que estamos trabalhando com o conjunto $\mathcal{L}$, temos

$$
\begin{aligned}
& \frac{\partial \varphi_{i}}{\partial x_{1}}(1,0)=0 \\
& \frac{\partial g}{\partial x_{1}}(u, 0)=\frac{\partial F}{\partial x_{1}}(1,0)+\sum_{1}^{M} u_{i} \underbrace{\frac{\partial \varphi_{i}}{\partial x_{1}}(1,0)}_{=0}=\frac{\partial f}{\partial x_{1}}(0)
\end{aligned}
$$

Por (i) temos que

$$
\begin{aligned}
& \frac{\partial h}{\partial u_{i}}(u, \alpha)=-\frac{\partial g}{\partial u_{i}}(u, h(u, \alpha), \alpha)\left(\frac{1}{\frac{\partial g}{\partial x_{1}}(u, h(u, \alpha), \alpha)}\right)= \\
& =-\frac{\partial g}{\partial u_{i}}(u, h(u, \alpha), \alpha)\left(\frac{1}{\frac{\partial F}{\partial x_{1}}(1, x)}\right)
\end{aligned}
$$

Assim, na diferença temos:

$$
\begin{aligned}
& \frac{\partial}{\partial u_{i}}(H(u, \beta, 0)-H(u, 0,0))=\frac{\partial f}{\partial x_{1}}(0)\left(-\frac{\partial g}{\partial u_{i}}(u, h(u, \beta), \beta) \frac{1}{\frac{\partial F}{\partial x_{1}}(1, x)}\right)= \\
& =-\frac{\frac{\partial f}{\partial x_{1}}(0)}{\frac{\partial F}{\partial x_{1}}(1, x)}\left(\frac{\partial g}{\partial u_{i}}(u, h(u, \beta), \beta)\right)
\end{aligned}
$$

Olhando para $\frac{\partial g}{\partial u_{i}}(u, h(u, \beta), \beta)$ e fazendo $u=0$, obtemos um polinômio em $\beta$ :

$$
\frac{\partial g}{\partial u_{i}}(0, h(0, \beta), \beta)=\varphi_{i}(\beta)
$$

Uma vez que $-\frac{\frac{\partial f}{\partial x_{1}}(0)}{\frac{\partial F}{\partial x_{1}}(1, x)}$ é um elemento invertível, o rank $\left\{j^{d}\left(\frac{\partial g}{\partial u_{i}}\right)\right\}_{u}$ coincide com o rank $\left\{\frac{\partial g}{\partial u_{i}}\right\}$, e segue-se então que $T_{0} \eta$ é sobre.

Assim, $\eta$ é uma submersão em alguma vizinhança $U \times A_{1} \times A_{2}$ de $0 \in K^{M} \times K^{n} \times$ 
$K^{n}$. Tomemos $U, A_{1}, A_{2}$ vizinhanças fechadas, reais subanalíticas (por exemplo, bolas fechadas), e $U^{\prime}$ uma vizinhança aberta de $U \times A_{1} \times A_{2}$ na qual $\eta$ é uma submersão. $\eta: U^{\prime} \rightarrow J^{d}\left(K^{n}, K\right)$ é transversal à $\tilde{X}$, então $\eta^{-1}(\tilde{X}) \cap U^{\prime}$ é uma subvariedade diferenciável de $U^{\prime}$. Seja $\Pi: K^{M} \times K^{n} \times K^{n} \rightarrow K^{M}$ a projeção canônica e $\Sigma o$ conjunto dos pontos singulares de $\left.\Pi\right|_{\eta^{-1}(\tilde{X}) \cap U^{\prime}}$.

Como $\eta$ e $\Pi$ são aplicações analíticas e para $K=\mathbb{R}, \bar{X}$ é semialgébrico (para $K=C$, $\bar{X}$ é construtivel) $\Sigma$ é semi-algébrico.

I : $U \times A_{1} \times A_{2} \rightarrow U$ é própria então $B=\Pi\left(\Sigma \cap\left(U \times A_{1} \times A_{2}\right)\right)$ é real subanalítico. Este conjunto tem, pelo teorema de Sard, medida nula e consequentemente possui codimensão $\geq 1$.

Segue do lema 1.4.1 que para $u \in U-B$ a aplicação $\eta_{u}: A_{1} \times A_{2} \rightarrow J^{d}\left(K^{n}, K\right)$ é transversal a $\tilde{X}$, então para todo $\alpha \in A_{1}$ o ponto $y=(h(u, \alpha), \alpha) \in\left\{g_{u}=0\right\}$ satisfaz a condição de tangente transversa dada na definição acima. Podemos escolher a vizinhança $A$ de $x(0)=(1,0, \ldots, 0)$, de forma que $(h, 1)^{-1}(A) \subset U \times A_{1}$.

Corolário 3.2.1 Seja $f$-determinada com número de Milnor $\mu$ (então $k \leq \mu+1$ ). Se $d \geq k$ então para quase toda hipersuperfície $\{G=0\}=V \subset \mathbb{P}^{n+1}$ de grau $d$, $o$ conjunto $V(f)=\{x \in V$; a função altura normal à $x$ possui uma singularidade $\mathcal{A}$-equivalente à $f\}$ é regular e tem dimensão $n+1-\mu$.

Exemplo 3 (i) $n=1$. Se $d \geq 3$, as únicas singularidades tangentes são as simples $\left(A_{1}\right)$ e cúspide $\left(A_{2}\right)$. (Os casos $d=1,2$ são triviais)

(ii) $n=2$. Se $d \geq 4$, as únicas singularidades tangentes são as simples $\left(A_{1}\right)$ (dois tipos no caso real), cúspide $\left(A_{2}\right)$ e tacnode $\left(A_{3}\right)$ (dois tipos no caso real).

(Novamente os casos $d=1,2$ são fáceis. Vamos argumentar o caso $d=3: A_{1}, A_{2}$ são 3-determinadas e a órbita do 2-jato $x^{2} \in J^{3}(2,1)$ tem codimensão 2 . As outras órbitas possuem codimensão $>2$. Então para $G$ tangente transversa às órbitas singulares de $J^{3}(2,1)$ obtemos tangências $\left(A_{1}\right)$ em um conjunto aberto, tangências cuspidais $\left(A_{2}\right)$ 
em uma curva e pontos isolados onde a função altura tem 2 -jato $x^{2}$. Mas como $\{G=$ 0\} é uma cúbica não singular, qualquer seção plana pode ter apenas singularidades do tipo $A_{1}, A_{2}, A_{3}, D_{4}$, então os pontos isolados são do tipo $A_{3}$ ).

\subsection{O teorema de genericidade multilocal}

Embora o teorama 3.2.1 acima descreva os tipos de singularidades tangentes que ocorrem em hipersuperfícies genéricas de grau suficientemente alto e este teorema seja suficiente para a discussão dos respectivos duais, para o caso de multitransversalidade vamos precisar mostrar que os pontos de tangência genericamente estão em posição geral nos hiperplanos tangentes.

Definição 3.3.1 Dados $K$ pontos $p_{1}, \ldots, p_{k} \in \mathbb{P}^{n}$, dizemos que eles estão em posição geral se as retas correspondentes em $\mathbb{C}^{n+1}$ formam subespaços linearmente independentes, então em particular $k \leq n+1$. Vamos denotar por $L\left(p_{1}, \ldots, p_{k}\right)$ os $(k-1)$-planos em $\mathbb{P}^{n}$ gerados por $p_{1}, \ldots, p_{k}$. O ponto $p_{0} \in L\left(p_{1}, \ldots, p_{k}\right)$ está em posição geral com relação a $p_{1}, \ldots, p_{k}$ se $p_{0}$ não está em nenhum subespaço determinado por um subconjunto próprio de $\left\{p_{1}, \ldots, p_{k}\right\}$.

Definição 3.3.2 Uma hipersuperfície $\{F=0\} \subset \mathbb{P}^{n+1}$ tem tangências em posição geral se dado um hiperplano $L$ tangente em $\left\{p_{1}, \ldots, p_{k}\right\} \subset\{F=0\}$ os pontos $p_{1}, \ldots, p_{k}$ estão em posição geral $L$.

Proposição 3.3.1 $O$ conjunto $X_{1}(k)=\{F ; F=0$ possui $k$ singularidades em posição geral\} é um conjunto construtivel de codimensão $k$ cujo fecho é irredutivel. 
Demonstração: $S e e_{i}$ denota o $i$-ésimo vértice de referência em $\mathbb{P}^{n+1}$, ou seja, $e_{i}=$ $(0, \ldots, 0,1,0, \ldots, 0)$ com 1 na i-ésima posição, o subconjunto de $\mathbb{C}^{M}$ consistindo das hipersuperfícies com singularidades em $e_{1}, \ldots, e_{k} \ell$ um espaço linear $L$, pois este subconjunto consiste de hipersuperfícies com singularidades nos mesmos pontos.

Se $\varphi: G L_{n+2} \times \mathbb{C}^{M}$ é a ação do grupo $G L_{n+2}=G L(n+2, \mathbb{C}$ ) (mudanças de coordenadas lineares na fonte), então $X_{1}=\varphi\left(G L_{n+2} \times L\right)$ é construtivel pelo teorema de Chevalley [3.1.1]. Se pensarmos em $G L_{n+2} \subset \mathbb{C}^{(n+2)^{2}}$ então existe uma extensão natural da ação de $\varphi$ em $\mathbb{C}^{(n+2)^{2}}$, e é claro que

$$
\overline{\varphi\left(G L_{n+2} \times L\right)} \subset \overline{\varphi\left(\overline{G L_{n+2}} \times L\right)}
$$

(onde a barra denota o fecho com respeito à topologia de Zariski).

Temos que $\overline{G L_{n+2}}$ e L são irredutíveis pois, $\overline{G L_{n+2}}=M_{n+2}$ o qual é irredutivel e $L$ é um espaço linear. Então $\overline{\varphi\left(G L_{n+2} \times L\right)}$ e $\overline{\varphi\left(\overline{G L_{n+2}} \times L\right)}$ são irredutiveis. Claramente $G L_{n+2} \times L \subset \overline{G L_{n+2}} \times L$ é aberto Zariski. Vamos considerar o seguinte diagrama:

$$
\begin{aligned}
G L_{n+2} \times L & \longrightarrow G L_{n+2} \times \mathbb{C}^{M} \\
(g, F) & \stackrel{\eta}{\rightarrow}(g, F \circ g) \\
\varphi & \searrow \downarrow \pi_{2} \\
& F \circ g
\end{aligned}
$$

onde $\varphi=\pi_{2} \circ \eta$.

Segue do teorema de Chevalley, que $\varphi\left(G L_{n+2} \times L\right)$ é construtivel. Note que $G L_{n+2}$ é um conjunto construtivel pois, seu complementar $\left(G L_{n+2}\right)^{c}$ é o conjunto das matrizes 
de determinante nulo, que é portanto um conjunto algébrico fechado.

$$
G L_{n+2}=\underbrace{M_{n+2}}_{\text {conj.alg.fechado }}-\underbrace{\left(G L_{n+2}\right)^{c}}_{\text {conj.alg.fechado }}
$$

Assim, segue que

$$
\eta\left(G L_{n+2} \times L\right)=\underbrace{G L_{n+2}}_{\text {construtivel }} \times \underbrace{\varphi\left(G L_{n+2} \times L\right)}_{\text {construtivel }} \text { é construtivel }
$$

Pelo teorema 3.1.2, $\varphi\left(G L_{n+2} \times L\right)$ contém um subconjunto aberto Zariski de $\overline{\varphi\left(G L_{n+2} \times L\right)}$, o que implica que $\operatorname{dim}\left(\varphi\left(G L_{n+2} \times L\right)\right)=\operatorname{dim}\left(\overline{\varphi\left(G L_{n+2} \times L\right)}\right)$. Por outro lado, temos

$$
\begin{aligned}
& \underbrace{G L_{n+2} \times L}_{Z \text {-aberto }} \subset \overline{G L_{n+2}} \times L \\
& \overline{G L_{n+2}} \times L \text { é construtivel }
\end{aligned}
$$

Assim pelo teorema 3.1.1, $\varphi\left(\overline{G L_{n+2}} \times L\right)$ é construtivel $\Rightarrow G L_{n+2} \times \varphi\left(\overline{G L_{n+2}} \times L\right)$ é construtível, pelo teorema 3.1.2, $\varphi\left(\overline{G L_{n+2}} \times L\right)$ contém um aberto da topologia de Zariski em $\overline{\varphi\left(\overline{G L_{n+2}} \times L\right)}$, portanto

$$
\operatorname{dim}\left(\varphi\left(\overline{G L_{n+2}} \times L\right)\right)=\operatorname{dim}\left(\overline{\varphi\left(\overline{G L_{n+2}} \times L\right)}\right)
$$

Assim,

$$
\overline{\varphi\left(G L_{n+2} \times L\right)}=\overline{\varphi\left(\overline{G L_{n+2}} \times \bar{L}\right)}
$$

o qual já vimos que é irredutível.

Para concluir a demonstração, precisaríamos ainda verificar que a codimensão de $X_{1}(k)$ é exatamente $k$. Vamos fazer com detalhes o caso $n+2=2$

Considere $X_{1}(k)=\left\{F \in \mathbb{C}^{M} ; F=0\right.$ tem $k$ singularidades em posição geral \}$, k=1,2$ 
(i) $k=1$. Mostremos que $\operatorname{cod}\left(X_{1}(1)\right)=1$. Seja $F\left(x_{1}, x_{2}\right)=a_{0} x_{1}^{d}+a_{1} x_{1}^{d-1} x_{2}+$ $\ldots+a_{d} x_{2}^{d}$, onde $F \in X_{1}(1)$.

$O$ fato de $F$ possuir um ponto singular implica que $F$ possui uma raiz de multiplicidade 2. Com efeito, todo polinômio homogêneo de grau d $F\left(x_{1}, x_{2}\right)$ em $\mathbb{C}\left[x_{1}, x_{2}\right]$ se decompõe em fatores lineares, isto é, $F\left(x_{1}, x_{2}\right)=\prod_{i=1}^{d}\left(a_{i} x_{1}+b_{i} x_{2}\right)$. Se os fatores são distintos então $x_{1}=x_{2}=0$ é a única singularidade de $F=0$. Sem perda de generalidade, podemos supor que $x_{2}=0$ não é solução de $F=0$. Assim, podemos fazer a nossa análise para o polinômio

$$
F\left(x_{1}, 1\right)=a_{0} x_{1}^{d}+a_{1} x_{1}^{d-1}+\ldots+a_{d}
$$

$O$ fato de $F$ ter uma raiz multipla nos diz que $p\left(a_{0}, \ldots, a_{d}\right)=0$, onde $p$ é a resultante entre $F\left(x_{1}, 1\right)$ e $\frac{\partial F}{\partial x_{1}}$. Assim, $X_{1}(1)$ pode ser reescrito da seguinte forma:

$X_{1}(1)=\left\{F \in \mathbb{C}^{M} ; p\left(a_{0}, \ldots, a_{d}\right)=0\right\}$, onde $p$ é a resultante entre $F$ e $\frac{\partial F}{\partial x_{1}}$, ambos desomogenizados com relação a $x_{2}$.

Note que $p\left(a_{0}, \ldots, a_{d}\right)=0$ define uma hipersuperficie em $\mathbb{C}^{M}$, cuja parte regular é determinada exatamente pelas equações:

$$
\left\{\begin{array}{l}
p=0 \\
\operatorname{grad}(p) \neq 0
\end{array}\right.
$$

Portanto $\operatorname{cod}\left(X_{1}(1)\right)=1$.

(ii) $k=2$. Mostremos que $\operatorname{cod}\left(X_{1}(2)\right)=2$.

Seja $F\left(x_{1}, x_{2}\right)=a_{0} x_{1}^{d}+a_{1} x_{1}^{d-1} x_{2}+\ldots+a_{d} x_{2}^{d}$, onde $F \in X_{1}(2)$. $O$ fato de $F$ possuir uma singularidade nos permite escrevê-la da seguinte maneira:

$$
\begin{aligned}
& F\left(x_{1}, x_{2}\right)=\underbrace{\left(\alpha_{1} x_{1}+\alpha_{2} x_{2}\right)^{2}}_{(*)} G_{1}\left(x_{1}, x_{2}\right) \\
& (*)=\text { parte determinada por uma das singularidades }
\end{aligned}
$$


Agora, analisando $G_{1}\left(x_{1}, x_{2}\right)$ de forma análoga ao caso $(i)$, obteremos uma equação $p_{1}=0$ a qual pode ser escrita em termos de $a_{0}, \ldots, a_{d}$, onde $p_{1}$ é a resultante entre $G_{1} e \frac{\partial G_{1}}{\partial x_{1}}$.

Repetindo o processo, colocando desta vez a parte relativa a outra singularidade em evidência, obteremos:

$$
F\left(x_{1}, x_{2}\right)=\left(\beta_{1} x_{1}+\beta_{2} x_{2}\right)^{2} G_{2}\left(x_{1}, x_{2}\right)
$$

Novamente, a partir de $G_{2}$, obtemos uma equação $p_{2}=0$, onde $p_{2}$ é a resultante entre $G_{2}$ e $\frac{\partial G_{2}}{\partial x_{1}}$.

Assim, a melhor situação ocorre quando $p_{1}=0$ intercepta $p_{2}=0$ transversalmente, o que corresponde a termos duas singularidades em posição geral.

$$
X_{1}(2)=\left\{F \in \mathbb{C}^{M} ;\left(\begin{array}{c}
p_{1}=0 \\
\operatorname{grad}\left(p_{1}\right) \neq 0
\end{array}\right) \cap\left(\begin{array}{c}
p_{2}=0 \\
\operatorname{grad}\left(p_{2}\right) \neq 0
\end{array}\right) \text { ocorre transversalmente }\right\}
$$

Portanto $\operatorname{cod}\left(X_{1}(2)\right)=2$.

Proposição 3.3.2 Seja $X_{2}$ o subconjunto de $X_{1}$, definido na proposição anterior, que consiste das hipersuperfícies que possuem uma singularidade adicional que está $n o(k-1)$-plano gerado pelas $k$ anteriores, e está em posição geral a elas. Afirmamos que $X_{2}$ é construtivel de codimensão $\geq k+1$

Demonstraçâo: A construtibilidade segue como na proposição anterior. Para mostrar que $X_{2}$ possui codimensão maior do que $X_{1}$, afirmamos que é suficiente mostrar que $X_{1}-X_{2}$ tem codimensão $k$. De fato, inicialmente vamos assumir que $\operatorname{cod}\left(X_{1}-X_{2}\right)=$ k. A hipótese de construtibilidade de um conjunto $X$ nos garante que $\operatorname{dim}(\bar{X}-X)<$ $\operatorname{dim}(X)=\operatorname{dim}(\bar{X})$. 
Agora, suponha que $\operatorname{dim}\left(X_{1}\right)=\operatorname{dim}\left(X_{2}\right)$, então $\operatorname{dim}\left(\overline{X_{1}}\right)=\operatorname{dim}\left(\overline{X_{2}}\right)$, como $X_{1}$ é irredutivel segue que $\overline{X_{1}}=\overline{X_{2}}$, e então, $\overline{X_{1}}-X_{2}=\overline{X_{2}}-X_{2}$. Então

$$
\begin{aligned}
& N-k=\operatorname{dim}\left(X_{1}-X_{2}\right) \leq \operatorname{dim}\left(\overline{X_{1}}-X_{2}\right)=\operatorname{dim}\left(\overline{X_{2}}-X_{2}\right)<\operatorname{dim}\left(\overline{X_{2}}\right)= \\
& =\operatorname{dim}\left(\overline{X_{1}}\right)=\operatorname{dim}\left(X_{1}\right)=N-k
\end{aligned}
$$

o que é absurdo. Então dado que $\operatorname{cod}\left(X_{1}-X_{2}\right)=k$ segue que $\operatorname{cod}\left(X_{2}\right)>k$.

$O$ fato de que $\operatorname{cod}\left(X_{1}-X_{2}\right)=k$ segue da proposição 3.3.1.

Proposição 3.3.3 Dado qualquer $k, 1 \leq k \leq n+2$, existem hipersuperfícies $F \in \mathbb{C}^{N}$ $\operatorname{com}\{F=0\}$ tendo $k$ singularidades em posição geral, e nenhuma outra singularidade.

Demonstração: Usaremos indução em $n$. Para $n=0$ as formas

$$
\left.F=x_{1}^{2} \prod_{j=1}^{d-2}\left(x_{1}-j x_{2}\right) \quad \text { (respectivamente, } F=x_{1}^{2} x_{2}^{2} \prod_{j=1}^{d-4}\left(x_{1}-j x_{2}\right)\right)
$$

possuem raizes repetidas em 1 (respectivamente 2) pontos.

$$
\begin{aligned}
& \text { Para } n=1 \text { tomaremos } d=3 \text { e consideremos } \\
& \left.\qquad F=x_{1} x_{2} x_{3}+x_{1}^{3}+x_{2}^{3} \quad \text { (respectivamente, } F=x_{3}\left(x_{3}^{2}-x_{1} x_{2}\right), F=x_{1} x_{2} x_{3}\right)
\end{aligned}
$$

Estas $F$ 's possuem respectivamente uma, duas e três singularidades em posição geral.

Agora, desejamos produzir hipersuperfícies $\{F=0\} \subset \mathbb{P}^{n+1}$ tendo apenas $k$ singularidades em posição geral, $1 \leq k \leq n+2$. Por indução podemos encontrar $G_{k}\left(x_{1}, \ldots, x_{n+1}\right)$ com $\left\{G_{k}=0\right\} \subset \mathbb{P}^{n}$ tendo apenas $k$ singularidades em posição geral, $1 \leq k \leq n+1$. A forma $G_{k}+x_{n+2}^{d}$ cuida dos casos $1 \leq k \leq n+1$. Para $k=n+2$ afirmamos que

$$
G_{n+2}=G_{n+1}+x_{n+2}^{d-2}\left(\sum^{n+1} a_{i} x_{i}^{2}\right)
$$


para $\left(a_{1}, \ldots, a_{n+1}\right)$ genéricos possui precisamente $n+2$ singularidades. Basta mostrarmos que

$$
\left\{G_{n+1}+\sum^{n+1} a_{i} x_{i}^{2}=0\right\} \subset \mathbb{C}^{n+1}
$$

possui apenas uma singularidade (na origem) para $\left(a_{1}, \ldots, a_{n+1}\right)$ genéricos. Deste modo, o ponto $(0, \ldots, 0,1)$ será uma singularidade de $\{F=0\} \subset \mathbb{P}^{n+1}$, conseguindo assim, as $n+2$ singularidades em posição geral desejadas.

Considere a aplicação

$$
G:\left(\mathbb{C}^{n+1}-0\right) \times \mathbb{C}^{n+1} \rightarrow \mathbb{C}, \quad(x, a) \mapsto G_{n+1}(x)+\sum a_{i} x_{i}^{2}=G_{a}(x)
$$

Note que $G$ é uma aplicação regular, de fato, calculando o gradiente de $G$ temos que:

$$
\nabla G=(\frac{\partial G_{n+1}}{\partial x_{1}}(x)+2 x_{1}, \ldots, \frac{\partial G_{n+1}}{\partial x_{n+1}}(x)+2 x_{n+1}, x_{1}^{2}, \ldots, \underbrace{x_{i}^{2}}_{\neq 0}, \ldots, x_{n+1}^{2})
$$

como estamos trabalhando em $\mathbb{C}^{n+1}$ - 0 temos que pelo menos um $x_{i} \neq 0,1 \leq i \leq$ $n+1 \Rightarrow \nabla G \neq 0$. Portanto, $G$ é regular. Pelo lema de Thom 1.4.1, temos que $G_{a}: \mathbb{C}^{n+1}-\{0\} \rightarrow \mathbb{C}$ é regular para quase todo $a \in \mathbb{C}^{n+1}$. Logo, $\left\{G_{a}=0\right\} \subset \mathbb{C}^{n+1}-0$ não possui pontos singulares, como desejávamos.

Teorema 3.3.1 O conjunto das F cujas tangências não estão em posição geral formam um conjunto construtivel de codimensão $\geq 1$.

Demonstração: Suponha que $\{F=0\}$ tem um plano tangente $T$ com $k+1$ pontos tangentes $p_{0}, \ldots, p_{k}$ com $p_{1}, \ldots, p_{k}$ em posição geral e $p_{0} \in L\left(p_{1}, \ldots, p_{k}\right)$ em posição geral com relação à $p_{1}, \ldots, p_{k}$. Começaremos considerando o caso especial $p_{i}=e_{i}, 1 \leq$ 
$i \leq k, p_{0}=(1, \ldots, 1,0, \ldots, 0)$ ( $k$ uns) e $T=\left\{x_{n+2}=0\right\}$, ou seja, $\frac{\partial F}{\partial x_{i}}\left(p_{j}\right)=0$ para qualquer $i=1, \ldots, n+1$ e $\frac{\partial F}{\partial x_{n+2}}\left(p_{j}\right)=1$. Então podemos escrever

$$
F=f\left(x_{1}, \ldots, x_{k}\right)+\sum_{k+1}^{n+2} x_{j} f_{j}\left(x_{1}, \ldots, x_{n+2}\right)
$$

Temos,

$$
\begin{aligned}
& F=0 \\
& \frac{\partial F}{\partial x_{i}}=\frac{\partial f}{\partial x_{i}}+\sum_{k+1}^{n+2} x_{j} \frac{\partial}{\partial x_{i}} f_{j}\left(x_{1}, \ldots, x_{n+2}\right)=0
\end{aligned}
$$

para $1 \leq i \leq k$ em $p_{0}, e_{1}, \ldots, e_{k}$. Em particular, $f=\frac{\partial f}{\partial x_{i}}=0$ nestes pontos.

Portanto $\mathbb{C}^{N} \subset \mathbb{C}^{M}$ é o conjunto das formas de grau d nas variáveis $x_{1}, \ldots, x_{k} e$ $X_{2}$ é o subconjunto de $\mathbb{C}^{N}$ discutido na proposição 3.3.2, assim, $f \in X_{2}$.

Além disso, $f_{j}=0$ em $e_{1}, \ldots, e_{k}$ para $k+1 \leq j \leq n+1$, pois continuando com as derivadas, $\frac{\partial F}{\partial x_{j}}\left(e_{l}\right)=f_{j}\left(e_{l}\right)$, como $\frac{\partial F}{\partial x_{j}}\left(e_{l}\right)=0$, pois o plano $x_{n+2}=0$ é tangente comum, isto é, $\operatorname{grad}\left(F\left(e_{l}\right)\right)=(0, \ldots, 0,1)$, segue que $f_{j}\left(e_{l}\right)=0, l=1, \ldots, k ; j=$ $k+1, \ldots n+1$.

Então, $f_{j}=\sum_{k+1}^{n+1} x_{i} f_{i j} e$

$$
F=f+\sum_{j=k+1}^{n+2} \sum_{i=k+1}^{n+1} x_{j} x_{i} f_{i j}
$$

$A$ coleção destas $F$ 's é um produto $X_{2} \times L$ para algum espaço linear $L$.

Mas estamos interessados na $\varphi\left(G L_{n+2}\left(X_{2} \times L\right)\right)$, mais ainda, queremos obter $\operatorname{cod}\left(\varphi\left(G L_{n+2}\left(X_{2} \times L\right)\right)\right)$. O espaço tangente a $X_{2} \times L$, satisfaz a:

$$
\tau=T_{(f, l)}\left(X_{2} \times L\right) \subset T_{f} X_{2}+S p\left\{x_{i} \psi ; \operatorname{grau}(\psi)=d-1, k+1 \leq i \leq n+2\right\}
$$

Com relação a $\varphi\left(G L_{n+2}\left(X_{2} \times L\right)\right)$ temos

$$
\begin{gathered}
G L_{n+2} \times\left(X_{2} \times L\right) \stackrel{\varphi}{\rightarrow} \mathbb{C}^{M} \\
(I, F) \longmapsto \varphi(I, F)
\end{gathered}
$$


Assim,

$d \varphi_{\left(I_{1} F\right)}(\tau)=\tau^{\prime}=T_{f}\left(\varphi\left(G L_{k} \times X_{2}\right)\right)+S p\left\{x_{i} \psi ;\right.$ grau $\left.\psi=d-1, k+1 \leq i \leq n+2\right\}+$ $+S p\left\{x_{i} \frac{\partial F}{\partial x_{j}} ; 1 \leq i, j \leq n+2\right\}$

Fazendo a interseção com $\mathbb{C}^{N}\left(x_{j}=0, k+1 \leq j \leq n+2\right)$

$$
\mathbb{C}^{N} \cap \tau^{\prime} \subset T_{f}\left(\varphi\left(G L_{k} \times X_{2}\right)\right)+S p\left\{x_{i} f_{n+2}\left(x_{1}, \ldots, x_{k}, 0, \ldots, 0\right), 1 \leq i \leq k\right\}
$$

(pois $f_{j}=0$ quando fazemos $x_{j}=0$ para $k+1 \leq j \leq n+1$ ).

Vimos na proposição 3.3.2 que $\operatorname{cod}\left(\varphi\left(G L_{k} \times X_{2}\right)\right)$ em $\mathbb{C}^{M}$ é maior que $k$. Portanto $\operatorname{cod}\left(\mathbb{C}^{N} \cap r^{\prime}\right)$ em $\mathbb{C}^{N} \dot{e} \geq 1$

Teorema 3.3.2 Seja $K=\mathbb{C}$. Seja $\{F=0\}$ uma hipersuperfície não singular com tangências em posição geral. Então dada uma uma estratificação natural $\chi \subset \Sigma \subset$ $J^{k}(n, 1)$, existe uma vizinhança aberta $U$ de $F$ e uma familia enumerável de subconjuntos reais subanalíticos $B_{\alpha} \subset U, \operatorname{com} \operatorname{cod}\left(B_{\alpha}\right) \geq 1$ tal que para $G \in U-\cup B_{\alpha} a$ hipersuperfície $\{G=0\}$ é tangente multitransversa a $\chi$ desde que $k \leq \frac{d-1}{2}$.

Antes de fazermos a demonstração, vamos dar uma idéia da prova:

Como de costume temos uma familia natural de perturbaçôes fornecida pela própria familia de hipersuperfícies. Suponha que temos pontos de tangência $x(1), \ldots, x(r)$ com um plano tangente comum $x_{0}=0$. Como os $x(j)$ estão em posição geral, vamos supor que eles são os pontos $e_{j+1}(1 \mathrm{na}(j+1)$-ésima posição e zero nas demais).

No caso em $r=1, x(1)=(0,1,0, \ldots, 0)$. Na demonstração deste caso (Teorema 3.2.1), vimos que apenas os monômios de grau $d$ em $x_{0}, \ldots, x_{n+1}$ que não envolvem $x_{0}$ ou o termo $x_{1}^{d}$ eram suficientes para mostrar que a aplicação $\eta$ em questão era uma submersão, ou seja, utilizamos apenas uma face do $(n+1)$ simplexo de monômios de grau $d$ em $x_{0}, \ldots, x_{n+1}$. 
No caso geral, desde que $k \leq \frac{d-1}{2}$, nós podemos em $e_{j}$, obter todos os monômios em $x_{1}, \ldots, x_{j-1}, x_{j+1}, \ldots, x_{n}$ de grau $\leq k$, sem usar qualquer monômio da face do simplexo duas vezes. De fato, seja $d_{i}$ o grau do monômio $x_{i}$, então temos que

$$
d_{j}+\left(d_{1}+\ldots+d_{j_{1}}+d_{j+1}+\ldots+d_{n}\right)=d
$$

queremos que $d_{1}+\ldots+d_{j_{1}}+d_{j+1}+\ldots+d_{n} \leq k$. Assim,

$$
d_{j} \geq d-k \geq d-\frac{d-1}{2}=\frac{d+1}{2}
$$

Logo, quando desomogenizamos com relação a uma outra variável, obteremos $d_{j}<$ $\frac{d+1}{2}$, deste modo não repetiremos nenhum monômio, como queríamos.

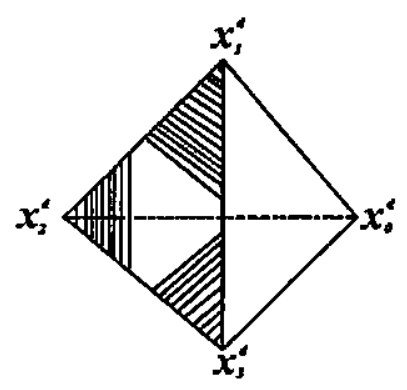

A principal diferença entre este caso e o caso $r=1$ é que $V^{(r)}$ não é compacto, apenas separável. Então quando estamos trabalhando localmente em $V^{(r)}$ precisamos sempre usar alguma vizinhança fixa $U$ de $F$. Isto significa (lembrando nossa demonstração do teorema 3.2.1) que dado $x \in V$, precisamos encontrar parametrizações $h_{u}$ válidas para $F_{u} \in U$. Como estamos trabalhando com multijatos, também queremos garantir que estas parametrizações locais não se interceptam.

\section{Demonstraçâo do teorema 3.3.2}

Afirmação: Podemos escolher $Q=(n+1)^{2}+1$ planos $L_{1}, \ldots, L_{Q}$ de modo que dados quaisquer $n+1$ pontos $p_{0}, \ldots, p_{n} \in \mathbb{P}^{n+1}$ um dos planos $L_{j}$ não contém nunhum ponto $p_{i}$. 
De fato, escolha os planos $L_{j}$ em posição geral (isto é, quaisquer $p \leq n+2$ planos fornecem $p$ planos em posição geral em $K^{n+2}$ ). Note que cada $p_{i}$ pertence a no máximo $n+1$ planos, como temos $n+1$ pontos, estes ocuparão no máximo $(n+1)^{2}$ planos, como $Q$ é superior a este número o resultado segue.

Agora, dado um hiperplano $L \subset \mathbb{P}^{n+1}$ temos que $\mathbb{P}^{n+1}-L \equiv K^{n+1}$. Escolha uma bola fechada $B_{j} \subset \mathbb{P}^{n+1}-L$ suficientemente grande de modo que $\mathbb{P}^{n+1}-B_{j}$ não contém $n+1$ pontos. Seja $V=\{F=0\}$, escolha $L_{j}$ e $B_{j}$ como acima mas com uma propriedade adicional de que a interseção de $V$ com a interseção de quaisquer $n+1$ complementos $\mathbb{P}^{n+1}-B_{j}$ é vazia, ou seja,

$$
\begin{aligned}
& V \cap\left(\cap_{n+1}\left(\mathbb{P}^{n+1}-B_{j}\right)\right)=\emptyset \\
& V \cap\left(\mathbb{P}^{n+1}-\cup_{n+1} B_{j}\right)=\emptyset \\
& \text { Logo, } V \subset \cup_{n+1} B_{j}
\end{aligned}
$$

Portanto, $\left(B_{j}\right)$ é uma cobertura de $V$.

Escolha uma vizinhança $U_{1}$ de $F$ de modo que $G \in U_{1}$ tem as mesmas propriedades que $F$ com relação as coberturas $B_{1}, \ldots, B_{Q}$ acima descritas. Escolha uma carta $x_{0}, \ldots, x_{n}$ em $\mathbb{P}^{n+1}-L_{j}$ e seja $B_{j} \cap V=V_{j}$ com equação $f_{j}=0$; seja

$$
N_{j}=\left\{(x, v) \in V_{j} \times K^{n+1} ; v=\lambda\left(\frac{\partial f_{j}}{\partial x_{0}}(x), \ldots, \frac{\partial f_{j}}{\partial x_{n}}(x)\right)\right\}
$$

e considere a aplicação $E: N_{j} \rightarrow K^{n+1}, E(x, v)=x+v$. Com a nossa notação $E$ é localmente a aplicação

$$
(\alpha, \lambda) \rightarrow h(\alpha)+\lambda\left(\frac{\partial f_{j}}{\partial x_{0}}(h(\alpha)), \ldots, \frac{\partial f_{j}}{\partial x_{n}}(h(\alpha))\right)
$$

e claramente $E$ é um difeomorfismo local. Usando o fato que $V_{j}$ é compacto escolha $\epsilon>0$ tal que a restrição $E: N_{j} \cap\left(V_{j} \times B_{\epsilon}\right) \rightarrow K^{n+1}$ é um mergulho, onde $B_{\epsilon} e^{\prime}$ uma bola de raio $\epsilon$ centrada em $0 \in K^{n+1}$. Agora escolha $U_{2} \subset U_{1}$ tal que para 
qualquer $G \in U_{2}$ o conjunto $W_{j}=B_{j} \cap\{G=0\}$ está contido na vizinhança $E\left(N_{j} \cap\right.$ $\left(V_{j} \times B_{\epsilon}\right)$ ).(Estritamente falando, devemos trabalhar com duas bolas grandes $B_{j}, B_{j}^{\prime}$ para evitarmos dificuldades na fronteira). Escolha um aberto $U \subset U_{2}$ com $\bar{U} \subset U_{2}$ compacto de modo que para $G \in \bar{U}$ e $y \in\{G=0\} \cap B_{j}$ com $y=E(x, v)$ o vetor $v$ não pertence a $T_{y} W_{j}$, isto é, $G$ é suficientemente próximo de $F$ para que os espaços tangentes também sejam próximos (Basta perceber que a vizinhança tubular de $G=$ 0 está contida na vizinhança tubular de $F=0$ e que perturbações suficientemente pequenas da $F$ vão dar perturbaçôes suficientemente pequenas de $\left.\left(\frac{\partial f_{j}}{\partial x_{0}}, \ldots, \frac{\partial f_{j}}{\partial x_{n}}\right)\right)$. Segue para qualquer $x \in V_{j}$ (o qual supomos ter espaço tangente $x_{0}=0$ ) podemos escolher uma vizinhança $A_{1}$ de $0 \in K^{n}$ e uma familia de parametrizações

$$
h: \bar{U} \times A_{1} \rightarrow K^{n+1}, h(u, \alpha)=\left(h^{\prime}(u, \alpha), \alpha\right)
$$

com $h_{u}$ parametrizando $F_{u}=0$. Mais ainda, como $h(\bar{U} \times 0) \subset\{E(h(0), v)\}$ dados $x(1), x(2) \in V_{j}$ com parametrizações locais $h_{j}: \bar{U} \times A_{1}^{j} \times K^{n+1}, j=1,2$,

$$
h_{1}(\bar{U} \times 0) \cap h_{2}(\bar{U} \times 0)=\emptyset
$$

então se necessário, podemos diminuir os $A_{1}^{j}$ e supor que $\bigcap_{j=1,2} h_{j}\left(U \times A_{1}^{j}\right)=\emptyset$.

Agora sejam $(x(1), \ldots, x(r)) \in\{F=0\}^{(r)}, r \leq n+1$ possuindo plano tangente comum e seja $L: K^{n} \rightarrow\left(K^{n+1}\right)^{*}$ como de costume. Escolha um $B_{j}$ com $x(i) \in$ $B_{j}, 1 \leq i \leq r$, e parametrizações locais $h_{j}: \bar{U} \times A_{1}^{j} \rightarrow K^{n+1}$ com

$$
h_{j}\left(U \times A_{1}^{j}\right) \cap h\left(U \times A_{1}^{i}\right)=\emptyset, i \neq j
$$

Considere a aplicação $\eta: A_{1}^{1} \times \ldots \times A_{1}^{r} \times U \times K^{n} \rightarrow \prod_{j=1}^{r} J^{k}\left(K^{n}, K\right)$

$$
\begin{aligned}
& \eta(\alpha(1), \ldots, \alpha(r), u, a)=\prod_{1}^{r}(k \text { jatos em } \alpha(i) d o s \\
& \alpha \rightarrow\left(d f_{j}(x(j))+L(a)\right)\left(h_{i}(u, \alpha(i))\right)= \\
& =\prod_{1}^{r}\left(j_{1}^{k} H_{i}(u, \alpha(i), a)\right)
\end{aligned}
$$

Afirmamos que $\eta$ é transversal a $\Delta^{r}(\chi)$. Se $j_{1}^{k} H_{i}(u, \alpha(i), a) \notin \Sigma$ para algum $i$, então

$$
\eta(\alpha(1), \ldots, \alpha(r), u, a) \notin \Delta^{r}(\chi)
$$


Se o $j_{1}^{k} H_{i}(u, \alpha(i), a) \in \Sigma$, mas os $h_{i}(u, \alpha(i))$ não possuirem um plano tangente comum, então

$$
\eta(\alpha(1), \ldots, \alpha(r), u, a) \notin \Delta^{r}(\chi)
$$

Contudo, se eles possuirem um plano tangente comum dado por

$$
\left(d f_{i}(\alpha(i))+L(a)\right)\left(h_{i}(u, \alpha(i))\right)=c
$$

então os $h_{i}(u, \alpha(i))$ estão em posição geral neste plano. Segue da observação acima que $\eta$ é uma submersão em $(\alpha(1), \ldots, \alpha(r), u, a)$ e assim, transversal a $\Delta^{r}(\chi)$. Então existe um conjunto ruim real subanalítico $B \subset U$ de codimensão $\geq 1$ tal que para $u \in U-B$ a aplicaçào

$$
\eta_{u}: \prod A_{1}^{j} \times K^{n} \rightarrow \prod J^{k}\left(K^{n}, K\right)
$$

é transverval a $\Delta^{r}(\chi)$. Agora, usamos o fato que $\{G=0\}^{(r)}$ é separável para obter uma quantidade enumerável de conjuntos ruins $B_{\alpha}$.

\subsection{Considerações finais}

Nesta última seção comentaremos alguns exemplos de cúspides da aplicação de Gauss projetiva. A principal referência é o artigo "Cusps of the projective Gauss map"[17].

Seja $M$ uma superfície algébrica regular em $\mathbb{P}^{3}$ tal que a familia $H$ é localmente versal. Sabemos que neste caso, a aplicação de Gauss $G: M \rightarrow \mathbb{P}^{3}$ é estável. Ou seja, as únicas singularidades que ocorrem são dobras e cúspides. Mais ainda, $\Sigma G$ é uma reunião disjunta de curvas, contendo pontos isolados que são os pontos de cúspide.

Em [17], Clint McCrory e Theodore Shifrin estudam as cúspides da aplicação 
de Gauss de superfícies em $\mathbb{P}^{3}$. Os principais resultados obtidos são os seguintes teoremas:

Teorema 3.4.1 Para uma superfície projetiva regular genérica mergulhada em $\mathbb{P}^{3}$, as cúspides da aplicação de Gauss são precisamente os pontos parabólicos os quais são os limites dos pontos de inflexão das curvas assintóticas.

$A$ demonstração deste teorema pode ser reduzida ao caso afim, caso em que o resultado é bastante conhecido. A demonstração apresentada pelos autores usa métodos da Geometria Diferencial projetiva.

Teorema 3.4.2 $A$ aplicação de Gauss de uma superfície projetiva genérica de grau $d$ em $\mathbb{P}^{3}$ possui $2 d(d-2)(11 d-24)$ cúspides.

A prova do teorema acima utiliza ferramentas da Teoria Enumerativa e escapa do alcance desta dissertação.

Nos exemplos de superfícies de grau $d=2,3$ é possível verificar diretamente $o$ resultado do teorema 3.4.2:

\section{Exemplo 4 Superfícies Quádricas}

Se $M \subset \mathbb{P}^{3}$ é uma quádrica genérica (ver apêndice)

$$
F\left(x_{1}, x_{2}, x_{3}, x_{4}\right)=0
$$

A equação dos pontos parabólicos da aplicação de Gauss é dada por

$$
\left\{\begin{array}{l}
F=0 \\
H \operatorname{ess}(F)=0
\end{array}\right.
$$


Como $F$ é homogênea de grau 2, Hess $(F)=$ constante, e portanto, vemos que genéricamente a aplicação de Gauss não possui singularidades.

Exemplo 5 Superfícies Cúbicas ([24], [25])

Seja $M$ uma cúbica genérica em $\mathbb{P}^{3}$. Segue do teorema 3.4 .2 que $M$ tem 54 cúspides, mas é possivel neste caso, argumentar diretamente.

Sabemos que existem 27 retas contidas em $M$. Evidentemente, cada reta é uma direçāo assintótica em $M$. Por outro lado, todos os pontos de uma tal reta $L \subset M$ são pontos de inflexão. Assim, aplicando o teorema 3.4.1, vemos que os pontos do conjunto parabólico $\Pi$ nos quais uma reta $L$ de $M$ é tangente a $\Pi$ correspondem aos pontos de cúspide da aplicação normal de Gauss $G$.

Não é difícil mostrar o seguinte resultado: "Cada reta de $M$ intercepta a curva parabólica em dois pontos e é tangente à essa curva nestes pontos".

De fato: Seja $L \subset M$ e $H$ um plano contendo $L$. Então $H \cap M$ é uma curva de grau 3 contendo $L$, logo $H \cap M=L \cup C$, onde $C$ é uma cônica.

Observemos que $C$ pode ser redutivel, mas $L \cup C$ não pode conter uma linha dupla, pois neste caso $M$ seria singular.

$O$ número de interseções de $L$ e $C$ é 2 (Teorema de Bezout [24]), e evidentemente $H$ é tangente a $M$ em cada um destes pontos $p_{i}, i=1,2$ de $L \cap C$

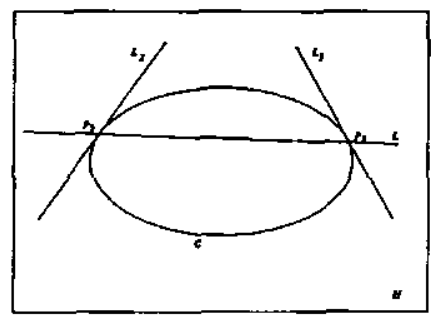


Se $L_{i}$ indica a reta tangente a $C$ em $p_{i}$, os pares de retas $\left(L, L_{1}\right)$ e $\left(L, L_{2}\right)$ são as direções assintóticas de $M$ em $p_{1}$ e $p_{2}$, respectivamente. (Estas são as retas com maior contato com $M$ em $\left.p_{i}\right)$. Quando estes dois pontos $p_{1}$ e $p_{2}$ coincidem, isto é, $L$ é tangente a $C$, o ponto $p=p_{1}=p_{2}$ é ponto parabólico de $M$ e $L$ é a única direção assintótica.

Agora definimos uma involução $\sigma$ de $L$ como segue. Dado $p \in L, T_{p} M \cap M$ é tangente a $M$ em outro ponto $q$, e definimos $\sigma(p)=q$. $A$ involução $\sigma$ é algébrica, $e$ pelo teorema do ponto fuxo de Lefschetz, $\sigma$ possui dois pontos fixos, os quais são os dois pontos parabólicos em $L$.

Exemplo 6 A cúbica de Fermat $x_{0}^{3}+x_{1}^{3}+x_{2}^{3}+x_{3}^{3}=0$ é um exemplo de uma superfície projetiva que não é genérica. A curva parabólica $\Pi$ é dada por $x_{0} x_{1} x_{2} x_{3}=0$. Os pontos singulares da curva $\Pi=0$ são obtidos a partir da solução dos determinantes dos menores $2 \times 2$ da matriz

$$
\left(\begin{array}{cccc}
3 x_{0}^{2} & 3 x_{1}^{2} & 3 x_{2}^{2} & 3 x_{3}^{2} \\
x_{1} x_{2} x_{3} & x_{0} x_{2} x_{3} & x_{0} x_{1} x_{3} & x_{0} x_{1} x_{2}
\end{array}\right)
$$

Note que o ponto $(1,0,0,0)$ é um ponto singular de $\Pi=0$. Portanto, $M$ não é uma superfície genérica.

Pode-se mostrar mais precisamente que $\Pi$ é uma curva redutível com 18 pontos singulares (pontos duplos) e que se perturbarmos a cúbica de Fermat cada um dos 18 pontos duplos vão dar origem a 3 cúspides da aplicação de Gauss. 


\section{Apêndice A}

\section{Geometria Algébrica Projetiva}

\section{A.1 O Espaço Projetivo e as Variedades Projetivas}

Definimos uma relação de equivalência $\sim$ em pontos não nulos de $K^{n+1}$ da seguinte forma: $\left(x_{0}^{\prime}, \ldots, x_{n}^{\prime}\right) \sim\left(x_{0}, \ldots, x_{n}\right)$ se existe um elemento não nulo $\lambda \in K$ tal que $\left(x_{0}^{\prime}, \ldots, x_{n}^{\prime}\right)=\lambda\left(x_{0}, \ldots, x_{n}\right)$

Definição A.1.1 Um espaço projetivo n-dimensional sobre um corpo $K$, denotado $\mathbb{P}^{n}(K)$, é o conjunto das classes de equivalência de $\sim$ em $K^{n+1}-\{0\}$. Assim,

$$
\mathbb{P}^{n}(K)=\left(K^{n+1}-\{0\}\right) / \sim
$$

Cada n-upla não nula $\left(x_{0}, \ldots, x_{n}\right) \in K^{n+1}$ define um ponto $p \in \mathbb{P}^{n}(K)$, e dizemos que $\left(x_{0}, \ldots, x_{n}\right)$ são as coordenadas homogêneas de $p$.

Existe uma correspôndencia $1-1$ entre pontos de $\mathbb{P}^{n}(K)$ e retas passando pela origem em $K^{n+1}$. De fato, todas as coordenadas homogêneas de $p \in \mathbb{P}^{n}(K)$ sāo 
dadas por $\lambda p, \lambda \in K-\{0\}$, e note que todos estes pontos pertencem a mesma reta passando pela origem em $K^{n+1}$. Como $p \neq 0$ temos a garantia de que esta reta existe. Por outro lado, dada qualquer reta $L$ passando pela origem em $K^{n+1}$, um ponto $p \in L-\{0\}$ nos fornece as coordenadas homogêneas de um único ponto em $\mathbb{P}^{n}(K)$.

Proposição A.1.1 Para cada $i=0, \ldots, n$, seja $U_{i}=\left\{\left(x_{0}, \ldots, x_{n}\right) \in \mathbb{P}^{n}(K) ; x_{i} \neq\right.$ $0\}$

(i) Os pontos de cada $U_{i}$ estão em correspondência 1-1 com os pontos de $K^{n}$.

(ii) $\mathbb{P}^{n}(K) \cong K^{n} \cup \mathbb{P}^{n-1}(K)$

(iii) Temos $\mathbb{P}^{n}(K)=\cup_{i=0}^{n} U_{i}$

\section{Demonstração:}

(i) Para cada $i=0, \ldots, n$ considere $\phi_{i}: K^{n} \rightarrow U_{i}$ dada por

$$
\phi_{i}\left(x_{1}, \ldots, x_{n}\right)=\left(x_{1}, \ldots, x_{i}, 1, x_{i+1}, \ldots, x_{n}\right)
$$

$e \psi_{i}: U_{i} \rightarrow K^{n}$ dada por

$$
\psi_{i}\left(x_{0}, \ldots, x_{n}\right)=\left(\frac{x_{0}}{x_{i}}, \ldots, \frac{x_{i-1}}{x_{i}}, \frac{x_{i+1}}{x_{i}}, \ldots, \frac{x_{n}}{x_{i}}\right)
$$

Primeiro devemos mostrar que $\psi_{i}$ está bem definida. Sejam $\left(x_{0}, \ldots, x_{n}\right)$ e $\left(y_{0}, \ldots, y_{n}\right)$ coordenadas homogêneas de $p \in U_{0}$. Assim temos que $\left(y_{0}, \ldots, y_{n}\right)=\lambda\left(x_{0}, \ldots, x_{n}\right)=$ $\left(\lambda x_{0}, \ldots, \lambda x_{n}\right)$. Então:

$\psi_{i}\left(y_{0}, \ldots, y_{n}\right)=\psi_{i}\left(\lambda x_{0}, \ldots, \lambda x_{n}\right)=\left(\frac{\lambda x_{0}}{\lambda x_{i}}, \ldots, \frac{\lambda x_{i-1}}{\lambda x_{i}}, \frac{\lambda x_{i+1}}{\lambda x_{i}}, \ldots, \frac{\lambda x_{n}}{\lambda x_{i}}\right)=$ $=\left(\frac{x_{0}}{x_{i}}, \ldots, \frac{x_{i-1}}{x_{i}}, \frac{x_{i+1}}{x_{i}}, \ldots, \frac{x_{n}}{x_{i}}\right)=\psi_{i}\left(x_{0}, \ldots, x_{n}\right)$.

Além disso,

$\phi_{i}\left(\psi_{i}\left(x_{0}, \ldots, x_{n}\right)\right)=\phi_{i}\left(\frac{x_{0}}{x_{i}}, \ldots, \frac{x_{i-1}}{x_{i}}, \frac{x_{i+1}}{x_{i}}, \ldots, \frac{x_{n}}{x_{i}}\right)=\left(\frac{x_{0}}{x_{i}}, \ldots, \frac{x_{i-1}}{x_{i}}, 1, \frac{x_{i}+1}{x_{i}}, \ldots, \frac{x_{n}}{x_{i}}\right)=$ $=\left(x_{0}, \ldots, x_{i-1}, x_{i}, x_{i+1}, \ldots, x_{n}\right)$ 
$\psi_{i}\left(\phi_{i}\left(x_{1}, \ldots, x_{n}\right)\right)=\psi_{i}\left(x 1, \ldots, x_{i}, 1, x_{i+1}, \ldots, x_{n}\right)=\left(\frac{x_{1}}{1}, \ldots, \frac{x_{i}}{1}, \frac{x_{i+1}}{1}, \ldots, \frac{x_{n}}{1}\right)=$ $=\left(x_{1}, \ldots, x_{n}\right)$

Portanto $\psi$ e $\phi$ são aplicações inversas, o. que estabelece a correspondência 1-1 desejada.

(ii) Pela definição de $U_{i}$, temos que

$$
\mathbb{P}^{n}(K)=U_{i} \cup H_{i}, \text { onde } H_{i}=\left\{p \in \mathbb{P}^{n}(K) ; i \text {-ésima coordenada é nula }\right\}
$$

Os pontos em $H_{i}$ estâo em correspondência 1-1 com as classes de equivalência $\left(x_{0}, \ldots, x_{i-1}, x_{i+1}, \ldots, x_{n}\right)$, onde duas n-uplas representam o mesmo ponto de $H_{i}$ se uma for um escalar não nulo multiplicado pela outra. Em outras palavras, $H_{i}$ é uma "cópia"de $\mathbb{P}^{n-1}(K)$, o espaço projetivo de dimensão menor.

$$
\mathbb{P}^{n}(K) \cong K^{n} \cup \mathbb{P}^{n-1}(K)
$$

(iii) Como cada $U_{i} \subset \mathbb{P}^{n}(K), 0 \leq i \leq n$, pela própria definição de $U_{i}$, segue que $\cup_{i=0}^{n} U_{i} \subset \mathbb{P}^{n}(K)$. Falta apenas mostrar que $\mathbb{P}^{n}(K) \subset \cup_{i=0}^{n} U_{i}$. Seja $p \in \mathbb{P}^{n}(K)$, logo, $p=\left(x_{0}, \ldots, x_{n}\right)$, onde $x_{0}, \ldots, x_{n}$ são não simultaneamente nulos, assim, $p \in U_{i}$ para algum $i$, portanto $p \in \cup_{i=0}^{n} U_{i}$.

Nosso próximo objetivo é estender a definição de variedades no espaço afim para o espaço projetivo.

Definição A.1.2 Seja $K$ um corpo e sejam $f_{1}, \ldots, f_{s}$ polinômios em $K\left[x_{1}, \ldots, x_{n}\right]$. Então o conjunto

$$
V\left(f_{1}, \ldots, f_{s}\right)=\left\{\left(a_{1}, \ldots, a_{n}\right) \in K^{n} ; f_{i}\left(a_{1}, \ldots, a_{n}\right)=0 ; \text { para todo } 1 \leq i \leq s\right\}
$$

é chamado de variedade afim definida por $f_{1}, \ldots, f_{s}$. 
Definição A.1.3 Um polinômio é homogêneo de grau d se todos os termos de $f$ possuem grau $d$.

Exemplo $7 f=x^{2}+2 x y+y^{2}$ é homogêneo de grau 2.

Observe que se $f \in K\left[x_{0}, \ldots, x_{n}\right]$ é um polinômio homogêneo que se anula em alguma coordenada homogênea de um ponto $p \in \mathbb{P}^{n}(K)$, então $f$ se anula em todas as coordenadas homogêneas de $p$.

Definição A.1.4 Seja $K$ um corpo e sejam $f_{1}, \ldots, f_{s} \in K\left[x_{0}, \ldots, x_{n}\right]$ polinômios homogêneos.

$$
V\left(f_{1}, \ldots, f_{s}\right)=\left\{\left(a_{0}, \ldots, a_{n}\right) \in \mathbb{P}^{n}(K) ; f_{i}\left(a_{0}, \ldots, a_{n}\right)=0 ; \text { para todo } 1 \leq i \leq s\right\}
$$

Chamamos $V\left(f_{1}, \ldots, f_{s}\right)$ a variedade projetiva definida por $f_{1}, \ldots, f_{s}$.

As variedades projetivas $V(f)$ definidas por uma única equação homogênea são conhecidas como hipersuperfícies. Estas são geralmente classificadas de acordo com o grau que definem suas equações. Assim se $f$ possui grau 2 em $K\left[x_{0}, \ldots, x_{n}\right]$, chamamos $V(f)$ de hipersuperfície quádrica ou quádrica.

Análogamente, hipersuperfícies definidas por equações de grau 3,4 e 5 são conhecidas como cúbicas, quárticas e quínticas respectivamente.

Proposição A.1.2 Seja $V=V\left(f_{1}, \ldots, f_{s}\right)$ uma variedade projetiva. Então $W=$ $V \cap U_{0}$ pode ser identificado com a variedade afim $V\left(g_{1}, \ldots, g_{s}\right) \subset K^{n}$, onde $g_{i}\left(y_{1}, \ldots, y_{n}\right)=$ $f_{i}\left(1, y_{1}, \ldots, y_{n}\right)$ para cada $1 \leq i \leq s$. 
Demonstração: Seja $p=\left(1, a_{1}, \ldots, a_{n}\right) \in W=V \cap U_{0}$. Usando a aplicação $\psi_{0}: U_{0} \rightarrow$ $K^{n}$ (da última proposição), temos que $\psi_{0}(p)=\left(a_{1}, \ldots, a_{n}\right)$ e como para cada $i$, temos $g_{i}\left(a_{1}, \ldots, a_{n}\right)=f_{i}\left(1, a_{1}, \ldots, a_{n}\right)=0$, segue que $\psi_{0}(p) \in V\left(g_{1}, \ldots, g_{s}\right)$. Por outro lado, se $\left(a_{1}, \ldots, a_{n}\right) \in V\left(g_{1}, \ldots, g_{s}\right)$, então o ponto com coordenadas homogêneas $\left(1, a_{1}, \ldots, a_{n}\right)$ pertence a $U_{0}$ e satisfaz as equações $f_{i}\left(1, a_{1}, \ldots, a_{n}\right)=g_{i}\left(a_{1}, \ldots, a_{n}\right)=$ 0. Assim, $\phi\left(V\left(g_{1}, \ldots, g_{n}\right)\right) \subset W$. Como as aplicações $\phi$ e $\psi$ são inversas, os pontos de $W$ estão em correspondência $1-1$ com os pontos de $V\left(g_{1}, \ldots, g_{s}\right)$.

Exemplo 8 Considere a variedade projetiva $V=V\left(x_{1}^{2}-x_{2} x_{0}, x_{1}^{3}-x_{3} x_{0}^{2}\right) \subset \mathbb{P}^{3}(R)$. Para interseccionar $V$ com $U_{0}$, nós desomogenizamos as equações que definem $V, o$ que dará a variedade afim $V\left(x_{1}^{2}-x_{2}, x_{1}^{3}-x_{3}\right) \subset R^{3}$.

Note que também podemos desomogenizar com relação à outras variáveis.

Uma idéia natural é reverter o processo de desomogenização descrito anteriomente $e$ "homogenizar" as equações que definem a variedade afim. Por exemplo, considere a variedade afim $W=V\left(x_{2}-x_{1}^{3}+x_{1}^{2}\right)$ em $U_{0}=R^{2}$. A equação que define $W$ não é homogênea, de modo que não obtemos uma variedade projetiva em $\mathbb{P}^{2}(R)$ diretamente desta equação. Mas podemos usar uma variável extra $x_{0}$ para tornar o polinômio $f=x_{2}-x_{1}^{3}+x_{1}^{2}$ homogêneo. Como $f$ possui grau 3, modificamos $f$ de modo que todos os seus termos possuam grau 3. Isto nos leva ao polinômio homogênio $f^{h}=$ $x_{2} x_{0}^{2}-x_{1}^{3}+x_{1}^{2} x_{0}$. Mais ainda, note que a desomogenização de $f^{h}$ nos devolve o polinômio original $f$ em $x_{1}, x_{2}$.

Agora, note que dada qualquer variedade afim $W=V\left(g_{1}, \ldots, g_{s}\right) \subset K^{n}$, podemos homogenizar as equações que definem $W$ de modo a obter a variedade projetiva $V=$ $V\left(g_{1}^{h}, \ldots, g_{s}^{h}\right) \subset \mathbb{P}^{n}(K)$. Mais ainda, $V \cap U_{0}=W$. Assim, nossa variedade afim original $W$ é a porção afim da variedade projetiva $V$. 
Exemplo 9 Neste exemplo, escreveremos as coordenadas homogêneas dos pontos em $\mathbb{P}^{2}(K)$ como sendo da forma $(x, y, z)$. Numerando estas coordenadas como $0,1,2$, vemos que $U_{2}$ é o conjunto dos pontos com coordenadas $(x, y, 1)$. Agora considere a variedade afim

$$
W=V(g)=V\left(y-x^{3}+x\right) \subset U_{2}
$$

Sabemos que $W$ é a porção afim $V \cap U_{2}$ da variedade projetiva $V=V\left(g^{h}\right)=$ $V\left(y z^{2}-x^{3}+x z^{2}\right)$. A variedade $V$ consiste de $W$ junto com os pontos no infinito $V \cap V(z)$. A porção afim $W$ é o gráfico de um polinômio cúbico, o qual é uma curva regular. Os pontos no infinito, os quais formam um complemento de $W$ em $V$, são dados pelas soluções das equações:

$$
\left\{\begin{array}{l}
y z^{2}-x^{3}+x z^{2}=0 \\
z=0
\end{array}\right.
$$

É fácil ver que as soluções são $z=x=0$ e como estamos trabalhando em $\mathbb{P}^{2}(K)$, tomamos o único ponto $p=(0,1,0)$ em $V \cap V(Z)$. Assim, $V=W \cup\{p\}$.

Vejamos o que acontece quando desomogenizamos a equação de $V$ com relação a $y$ e estudamos a interseç̧ão $V \cap U_{1}$.

$$
W^{\prime}=V \cap U_{1}=V\left(g^{h}(x, 1, y)\right)=V\left(z^{2}-x^{3}+x z^{2}\right)
$$

Através da teoria de singularidades, podemos facilmente verificar que $p$, que corresponde ao ponto $(x, z)=(0,0) \in W^{\prime}$, é um ponto singular de $W^{\prime}$.

Assim, mesmo começando com uma variedade afim suave, homogenizando as equações e tomando a correspondente variedade projetiva podemos obter um objeto geométrico mais complicado, pois, não estávamos "vendo toda a figura"na porção afim original da variedade. Em geral, dada uma variedade projetiva $V \subset \mathbb{P}^{n}(K)$, como $\mathbb{P}^{n}(K)=\cup_{i=0}^{n} U_{i}$, precisamos considerar $V \cap U_{i}$ para diversos valores de $i=0, \ldots, n$ para vermos toda a figura. 


\section{A.2 Correspondência entre Ideais Projetivos e Vari- edades Projetivas}

Definição A.2.1 (Ordem Lexicográfica); Seja $\alpha=\left(\alpha_{1}, \ldots, \alpha_{n}\right)$ e $\beta=\left(\beta_{1}, \ldots, \beta_{n}\right) \in$ $Z_{\geq 0}^{n}$. Dizemos que $\alpha>_{\text {lex }} \beta$ se no vetor diferença $\alpha-\beta \in Z_{\geq 0}^{n}$, o valor não nulo mais à esquerda é positivo. Escrevemos $x^{\alpha}>_{l e x} x^{\beta}$ se $\alpha>_{l e x} \beta$

Definição A.2.2 (Ordem Graduada Lex). Sejam $\alpha, \beta \in Z_{\geq 0}^{n}$. Dizemos que $\alpha>_{\text {grelex }}$ $\beta$ se $|\alpha|=\sum_{i=1}^{n} \alpha_{i}>|\beta|=\sum_{i=1}^{n} \beta_{i}$ ou,$|\beta|=|\alpha|$ e $\alpha>_{\text {lex }} \beta$.

Definição A.2.3 Seja $I \subset K\left[x_{1}, \ldots, x_{n}\right]$ um ideal não nulo.

(i) O termo de liderança de um polinômio $f(L T(f))$, é o monômio de $f$ que possui maior grau.

(ii) Denotamos por $L T(I)=\left\{c x^{\alpha}\right.$; existe $\left.f \in I \operatorname{com} L T(f)=c x^{\alpha}\right\}$

(iii) Denotamos por $\langle L T(I)>$ o ideal gerado pelos elementos de $L T(I)$

Definição A.2.4 Fixe uma ordem monomial. Um subconjunto finito $G=\left\{g_{1}, \ldots, g_{s}\right\}$ de um ideal I é uma base de Gröebner se

$$
<L T\left(g_{1}\right), \ldots, L T\left(g_{s}\right)>=<L T(I)>
$$

Definição A.2.5 Um ideal I em $K\left[x_{1}, \ldots, x_{n}\right]$ é homogêneo se para cada $f \in I$, as componentes homogêneas $f_{i}$ de $f$ também estiverem em $I$. 
Teorema A.2.1 Seja $I \subset K\left[x_{0}, \ldots, x_{n}\right]$ um ideal. Então são equivalentes:

(i) I é um ideal homogêneo de $K\left[x_{0}, \ldots, x_{n}\right]$

(ii) $I=<f_{1}, \ldots, f_{s}>$, onde $f_{1}, \ldots, f_{s}$ são polinômios homogêneos.

Demonstraçẫo: $(i i) \Rightarrow(i)$

Seja $g \in I$, então $g=\sum_{j=1}^{s} A_{j} f_{j}$. Agora suponha que expandimos cada $A_{j}$ como soma de suas componentes homogêneas: $A_{j}=\sum_{k=1}^{d} A_{j d}$. Portanto,

$$
g=\sum_{j=1}^{s} \sum_{k=1}^{d} A_{j k} f_{j}
$$

Podemos reagrupar os termos de g que possuem mesmo grau da seguinte forma:

$$
g=\sum_{j+k=2}^{s+d} A_{j k} f_{j}, 1 \leq j \leq d, 1 \leq k \leq d
$$

Note que as componentes homogêneas de $g$ pertencem à $I$, logo $I$ é um ideal homogêneo.

(i) $\Rightarrow$ (ii) Seja I um ideal homogêneo. Pelo Teorema das Bases de Hilbert, temos $I=<F_{1}, \ldots, F_{s}>$ para alguns polinômios $F_{j} \in K\left[x_{0}, \ldots, x_{n}\right]$ (não necessariamente homogêneos). Se escrevermos $F_{j}$ como a soma de suas componentes homogêneas, digamos $F_{j}=\sum_{i} F_{j i}$, então cada $F_{j i} \in I$ pois I é homogêneo. Seja $I^{\prime}$ o ideal gerado pelos polinômios homogêneos $F_{j i}$. Entâo $I \subset I^{\prime}$ pois cada $F_{j}$ é uma soma dos geradores de $I^{\prime}$. Por outro lado, $I^{\prime} \subset I$ pois cada componente homogênea de $F_{j}$ está em I. Isto mostra que $I=I^{\prime}$ e segue que I possui uma base de polinômios homogêneos.

Observação A.2.1 Seja $I \subset K\left[x_{0}, \ldots, x_{n}\right]$ um ideal homogêneo e suponha que $I=<f_{1}, \ldots, f_{s}>$, onde $f_{1}, \ldots, f_{s}$ são homogêneos. Então é fácil ver que

$$
V(I)=V\left(f_{1}, \ldots, f_{s}\right)
$$

de modo que $V(I)$ é uma variedade projetiva. 
Proposição A.2.1 Seja $V \subset \mathbb{P}^{n}(K)$ uma variedade projetiva e seja

$$
I(V)=\left\{f \in K\left[x_{0}, \ldots, x_{n}\right] ; f\left(a_{0}, \ldots, a_{n}\right)=0, \forall\left(a_{0}, \ldots, a_{n}\right) \in V\right\}
$$

(Isto significa que $f$ se anula para todas as coordenadas homogêneas de todos os pontos em $V)$.

Se $K$ é infinito, então $I(V)$ é um ideal homogêneo em $K\left[x_{0}, \ldots, x_{n}\right]$

Demonstração: Note que $I(V)$ é fechado com relação à soma e com relação ao produto por elementos de $K\left[x_{0}, \ldots, x_{n}\right]$.

Assim, $I(V)$ é um ideal em $K\left[x_{0}, \ldots, x_{n}\right]$. Agora, pegue $f \in I(V)$ e fixe um ponto $p \in V$. Por hipótese, $f$ se anula em todas as coordenadas homogêneas $\left(a_{0}, \ldots, a_{n}\right)$ de $p$.

Como $K$ é infinito, segue que cada componente homogênea $f_{i}$ de $f$ se anula em $\left(a_{0}, \ldots, a_{n}\right)$.

De fato, podemos escrever $f=\sum f_{i}$ como a soma de suas componentes homogêneas $f_{i}$

$$
f=f_{0}+f_{1}+\ldots+f_{n}
$$

Como $p \in V$ e $\left(a_{0}, \ldots, a_{n}\right)$ é coordenada homogênea de $p$ segue que

$$
\begin{aligned}
& f\left(a_{0}, \ldots, a_{n}\right)=f_{0}\left(a_{0}, \ldots, a_{n}\right)+f_{1}\left(a_{0}, \ldots, a_{n}\right)+\ldots+f_{n}\left(a_{0}, \ldots, a_{n}\right)=0 \\
& f\left(2 a_{0}, \ldots, 2 a_{n}\right)=f_{0}\left(a_{0}, \ldots, a_{n}\right)+2 f_{1}\left(a_{0}, \ldots, a_{n}\right)+\ldots+2^{n} f_{n}\left(a_{0}, \ldots, a_{n}\right)=0 \\
& \vdots \\
& f\left((n+1)\left(a_{0}, \ldots, a_{n}\right)\right)=f_{0}\left(a_{0}, \ldots, a_{n}\right)+(n+1) f_{1}\left(a_{0}, \ldots, a_{n}\right)+\ldots+ \\
& +(n+1)^{n} f_{n}\left(a_{0}, \ldots, a_{n}\right)=0
\end{aligned}
$$


Note que o nosso sistema é representado por

$$
\underbrace{\left(\begin{array}{cccc}
1 & 1 & \ldots & 1 \\
1 & 2^{1} & \ldots & 2^{n} \\
\vdots & & & \\
1 & (n+1)^{1} & \ldots & (n+1)^{n}
\end{array}\right)}_{\text {Matriz de Vandermonde } \Rightarrow \operatorname{det} \neq 0}\left(\begin{array}{c}
f_{0}\left(a_{0}, \ldots, a_{n}\right) \\
\vdots \\
f_{n}\left(a_{0}, \ldots, a_{n}\right)
\end{array}\right)=\left(\begin{array}{c}
0 \\
0 \\
\vdots \\
0
\end{array}\right)
$$

Logo o sistema possui solução única. Como $(0, \ldots, 0)$ é uma solução segue que

$$
\begin{aligned}
& f_{0}\left(a_{0}, \ldots, a_{n}\right)=0 \\
& \vdots \\
& f_{n}\left(a_{0}, \ldots, a_{n}\right)=0
\end{aligned}
$$

Isto mostra que $f_{i} \in I(V)$ e assim, $I(V)$ é homogêneo.

Teorema A: Seja K um corpo infinito. Então as aplicações

$$
\begin{aligned}
& \text { Variedades Projetivas } \stackrel{I}{\longrightarrow} \text { Ideais Homogêneos } \\
& \text { Ideais Homogêneos } \stackrel{V}{\longrightarrow} \text { Variedades Projetivas }
\end{aligned}
$$

são inclusões reversas. Além do mais, para qualquer variedade projetiva, temos $V(I(V))=V$ de modo que I é sempre $1-1$.

Demonstraçäo: Mostremos que I e $V$ são inclusões reversas:

(i) $\mathrm{Se} I_{1} \subset I_{2}$ então $V\left(I_{2}\right) \subset V\left(I_{1}\right)$

Seja $p$ um ponto pertencente à $V\left(I_{2}\right)$. Isto significa que para todo $f \in I_{2}, f$ se anula em todas as coordenadas homogêneas $\left(a_{0}, \ldots, a_{n}\right)$ de p. Como $I_{1} \subset I_{2} \Rightarrow \forall g \in I_{1}, g$ se anula em todas as coordenadas homogêneas $\left(a_{0}, \ldots, a_{n}\right)$ de $p \Rightarrow p \in V\left(I_{1}\right)$.

(ii) $V_{1} \subset V_{2}$ então $I\left(V_{2}\right) \subset I\left(V_{1}\right)$

Seja $f \in I\left(V_{2}\right)$, logo $f$ se anula em todas as coordenadas homogêneas de todos os pontos de $V_{2}$, como $V_{1} \subset V_{2}$ temos que $f$ se anula em todas as coordenadas homogêneas de todos os pontos de $V_{1} \Rightarrow f \in I\left(V_{1}\right)$ 
Resta mostrar que $V(I(V))=V$, quando $V=V\left(f_{1}, \ldots, f_{s}\right)$ é uma variedade projetiva. Como todo $f \in I(V)$ se anula em todas as coordenadas homogêneas dos pontos de $V$, a inclusão $V \subset V(I(V))$ segue diretamente da definição de $V$.

Por outro lado, note que $f_{1}, \ldots, f_{s} \in I(V)$ pela definição de $I$, e assim, < $f_{1}, \ldots, f_{s}>\subset I(V)$. Como $V$ é inclusão reversa, segue que $V(I(V)) \subset V\left(<f_{1}, \ldots, f_{s}\right\rangle$ )$=V$. Isto mostra a igualdade desejada $V(I(V))=V$ e consequentemente, $I$ é 1-1.

Definição A.2.6 O radical de um ideal é o ideal:

$$
\sqrt{I}=\left\{f \in K\left[x_{0}, \ldots, x_{n}\right] ; f^{n} \in I \text { para algum } n \geq 1\right\}
$$

Proposição A.2.2 Seja $I \subset K\left[x_{0}, \ldots, x_{n}\right]$ um ideal homogêneo. Então $\sqrt{I}$ também é um ideal homogêneo.

Demonstração: Se $f \in \sqrt{I}$, então $f^{m} \in I$ para algum $m \geq 1$. Agora, decompondo $f$ nas suas componentes homogêneas

$$
f=\sum_{i} f_{i}=f_{\max }+\sum_{i<\max } f_{i}
$$

onde $f_{\max }$ é a componente homogênea não nula de maior grau em $f$. Expandindo a potência $f^{m}$, é fácil ver que

$$
\left(f^{m}\right)_{\max }=\left(f_{\max }\right)^{m}
$$

Como I é um ideal homogêneo, $\left(f^{m}\right)_{\max } \in I$. Assim, $\left(f_{\max }\right)^{m} \in I$, o que mostra que $f_{\max } \in \sqrt{I}$. Se considerarmos $g=f-f_{\max } \in \sqrt{I}$ e repetirmos o argumento, teremos que $g_{\max } \in \sqrt{I}$. Mas $g_{\max }$ também é uma componente homogênea de $f$. Aplicando repetidamente este argumento, mostramos que todas as componentes homogêneas de $f$ estão em $\sqrt{I}$. Como isto vale para todo $f \in \sqrt{I}$, temos que $\sqrt{I}$ é um ideal homogêneo. 
Agora vamos recordar o Nullstellensatz Fraco e o Nullstellensatz Forte no caso afim:

Seja I um ideal contido em $K\left[x_{1}, \ldots, x_{n}\right]$

- (O Nullstellensatz Fraco) $V_{a}(I)=\emptyset$ em $K^{n} \Leftrightarrow I=K\left[x_{1}, \ldots, x_{n}\right]$

- (O Nullstellensatz Forte) $\sqrt{I}=I_{a}\left(V_{a}(I)\right)$ em $K\left[x_{1}, \ldots, x_{n}\right]$

(Para evitar confusão, usaremos $I_{a}$ e $V_{a}$ para denotar as versões afim de $I$ e $V$ ).

É natural questionarmos se estes resultados se estendem à variedades projetivas e ideais homogêneos.

A resposta é não. Em particular o Nullstellensatz Fraco falha para certos deais homogêneos. Por exemplo, considere o ideal $I=<x_{0}, \ldots, x_{n}>\subset C\left[x_{0}, \ldots, x_{n}\right]$.

Então $V(I) \subset \mathbb{P}^{n}(C)$ é definido pelas equações $x_{0}=\cdots=x_{n}=0$. A única solução é $(0, \ldots, 0)$, mas isto é impossivel, pois não é permitido que todas as coordenadas homogêneas se anulem simultâneamente.

Segue que $V(I)=\emptyset$ e ainda, $I \neq C\left[x_{0}, \ldots, x_{n}\right]\left(1+x_{0} \notin I\right)$.

Felizmente, é possivel caracterizar os ideais para os quais isto acontece.

A seguinte versão do Nulltellensatz Fraco descreve todos os ideais homogêneos sem soluções projetivas.

Teorema A.2.2 (O Nullstellensatz Fraco Projetivo) Seja $K$ algebricamente fechado e seja I um ideal homogêneo em $K\left[x_{0}, \ldots, x_{n}\right]$. Então são equivalentes:

(i) $V(I) \subset \mathbb{P}^{n}(K)$ é vazio

(ii) Para cada $0 \leq i \leq n$, existe um inteiro $m_{i} \geq 0$ tal que $x_{i}^{m_{i}} \in I$.

(iii) Existe algum $r \geq 1$ tal que $<x_{0}, \ldots, x_{n}>^{r} \subset I$ 
Demonstração: $(i i) \Rightarrow(i)$

Segue que $\left\langle x_{0}^{m_{0}}, \ldots, x_{n}{ }^{m_{n}}>\subset I\right.$, assim, aplicando $V$, temos que $V(I)$ está contido em $\left.V\left(<x_{0}{ }^{m_{0}}, \ldots, x_{n}{ }^{m_{n}}\right\rangle\right)=\emptyset$ pois estamos em $\mathbb{P}^{n}(K)$, logo $V(I)=\emptyset$.

(iii) $\Rightarrow(i i)$

É claro que se $\left\langle x_{0}, \ldots, x_{n}>^{r} \subset I\right.$ então $x_{i}^{r} \in N \forall$.

Para mostrar $(i) \Rightarrow\left(\right.$ iii), trabalharemos com a variedade afim $C_{v}=V_{a}(I) \subset K^{n+1}$, como interpretamos pontos em $\mathbb{P}^{n}(K)$ como retas passando pela origem em $K^{n+1}$, então $C_{v}$ é a união das retas determinadas por $V$. Em particular, $C_{v}$ contém todas as coordenadas homogêneas dos pontos de $V$.

Então, observe que se $V=\emptyset \Rightarrow C_{v} \subset\{(0, \ldots, 0)\}$ em $K^{n+1}$. Obtemos então

$$
I_{a}(\{(0, \ldots, 0)\}) \subset I_{a}\left(C_{v}\right)=I_{a}\left(V_{a}(I)\right)
$$

$\operatorname{Mas} I_{a}(\{(0, \ldots, 0)\})=<x_{0}, \ldots, x_{n}>$.

Pela versão afim do Nullstellensatz forte, temos que

$$
I_{a}\left(C_{v}\right)=I_{a}\left(V_{a}(I)\right)=\sqrt{I}
$$

Assim, $<x_{0}, \ldots, x_{n}>\subset \sqrt{I}$, logo existe $r \geq 1$ tal que $\left\langle x_{0}, \ldots, x_{n}>^{r} \subset I\right.$.

Teorema A.2.3 (O Nulltellensatz Forte Projetivo) Seja K um corpo algebricamente fechado e seja I um ideal homogêneo em $K\left[x_{0}, \ldots, x_{n}\right]$. Se $V=V(I)$ é uma variedade projetiva não vazia em $\mathbb{P}^{n}(K)$, então temos

$$
I(V(I))=\sqrt{I}
$$

Demonstração: Como na demostração do teorema anterior, trabalharemos com a variedade projetiva $V=V(I) \subset \mathbb{P}^{n}(K)$ e seu cone afim $C_{v}=V_{a}(I) \subset K^{n+1}$. Primeiro afirmamos que

(*) $I_{\mathbf{a}}\left(C_{v}\right)=I(V)$ quando $V \neq \emptyset$ 
Para ver isto, suponha que $f \in I_{a}\left(C_{v}\right)$. Dado $p \in V$, qualquer coordenada homogênea de $p$ pertence à $C_{v}$, de modo que $f$ se anula em todas as coordenadas homogêneas de $p$. Por definição, isto implica $f \in I(V)$.

Por outro lado, tome $f \in I(V)$. Como todo ponto não nulo de $C_{v}$ fornece coordenadas homogêneas para um ponto em $V$, segue que $f$ se anula em $C_{v}-\{0\}$. Resta mostrar que $f$ se anula na origem.

Como $I(V)$ é um ideal homogêneo, sabemos que as componentes homogêneas $f_{i}$ de $f$ também se anulam em $V$. Em particular, o termo constante de $f$, o qual é homogêneo na componente $f_{0}$ de grau zero, se anula em $V$. Como $V \neq 0$, isto força $f_{0}=0$,o que significa que $f$ se anula na origem. Assim, $f \in I_{a}\left(C_{v}\right)$ e $(*)$ está mostrado.

Pela forma afim do Nullstellensatz Forte, sabemos que $\sqrt{I}=I_{a}\left(V_{a}(I)\right)$. Então usando $(*)$, obtemos:

$$
\sqrt{I}=I_{a}\left(V_{a}(I)\right)=I_{a}\left(C_{v}\right)=I(V)=I(V(I))
$$

O que completa a demonstração do teorema.

Agora podemos completar a correspondência projetiva ideal-variedade.

Como no caso afim, temos uma correspondência 1-1 entre variedades projetivas $e$ ideais radicais homogêneos com excessão dos casos $\sqrt{I}=<x_{0}, \ldots, x_{n}>e \sqrt{I}=<$ $1>$.

Teorema A.2.4 Seja $K$ um corpo algebricamente fechado. Se restringirmos as correspondências do teorema $A$ a variedades projetivas não vazias e ideais radicais homogêneos própriamente contidos em $\left\langle x_{0}, \ldots, x_{n}>\right.$ então:

Variedades Projetivas não vazias $\stackrel{I}{\longrightarrow}$ Ideais Rad. Hom. própr. contidos em $\left\langle x_{0}, \ldots, x_{n}\right\rangle$ Ideais Rad. Hom. própr. contidos em $<x_{0}, \ldots, x_{n}>\stackrel{V}{\longrightarrow}$ Variedades Projetivas não vazias 
Demonstração: Note que uma consequência do Nullstellensatz Fraco projetivo é que os únicos ideais radicais homogêneos $I \operatorname{com} V(I)=\emptyset$ são $<x_{0}, \ldots, x_{n}>$ e $K\left[x_{0}, \ldots, x_{n}\right]$. Uma segunda observação é que se I é um ideal homogêneo diferente de $K\left[x_{0}, \ldots, x_{n}\right]$, então $I \subset<x_{0}, \ldots, x_{n}>$.

Estas observações mostram que os ideais radicais homogêneos com $V(I) \neq \emptyset$ são precisamente aqueles que satisfazem $I$ estritamente contido em $\left\langle x_{0}, \ldots, x_{n}\right\rangle$. E a relação segue do Nullstellensatz Forte projetivo.

Definição A.2.7 Seja I um ideal em $K\left[x_{1}, \ldots, x_{n}\right]$. Definimos a homogenização de I como sendo o ideal

$$
I^{h}=<f^{h} ; f \in I>\subset K\left[x_{0}, \ldots, x_{n}\right]
$$

onde $f^{h}$ é a homogenização de $f$.

Proposição A.2.3 Para qualquer ideal $I \subset K\left[x_{1}, \ldots, x_{n}\right]$, a homogenização $I^{h}$ é um ideal homogêneo em $K\left[x_{0}, \ldots, x_{n}\right]$

Demonstração: $O$ ideal $I^{h}$ possui um conjunto finito de geradores e pela própria definição de $I^{h}$, estes geradores são polinômios homogêneos. Logo $I^{h}$ é um ideal homogêneo.

Exemplo 10 Considere $\left.I=<f_{1}, f_{2}\right\rangle=\left\langle x_{2}-x_{1}^{2}, x_{3}-x_{1}^{3}\right\rangle$, o ideal da cúbica torcida afim. Se homogenizarmos $f_{1}, f_{2}$ então teremos o ideal $\left.J=<x_{2} x_{0}-x_{1}^{2}, x_{3} x_{0}^{2}-x_{1}^{3}\right\rangle$ em $R\left[x_{0}, x_{1}, x_{2}, x_{3}\right]$.

Afirmamos que $J \neq I^{h}$. Para mostrarmos isto, considere o polinômio $f_{3}=f_{2}-$ $x_{1} f_{1}=x_{3}-x_{1} x_{2} \in I \Rightarrow f_{3}^{h}=x_{0} x_{3}-x_{1} x_{3} \in I^{h}$. Agora note que $f_{3}^{h}$ não pode ser obtido como combinação de $f_{1}^{h}$ e $f_{2}^{h}$. Logo, $f_{3}^{h} \notin J \Rightarrow J \neq I^{h}$. 
Precisamos então, de um método para calcular um conjunto finito de geradores para o ideal $I$.

Teorema B: Seja I um ideal em $K\left[x_{1}, \ldots, x_{n}\right]$ e seja $G=\left\{g_{1}, \ldots, g_{s}\right\}$ uma base de Gröebner para I com respeito a uma ordenação monomial graduada em $K\left[x_{1}, \ldots, x_{n}\right]$. Então $G^{h}=\left\{g_{1}^{h}, \ldots, g_{s}^{h}\right\}$ é uma base para $I^{h} \subset K\left[x_{0}, \ldots, x_{n}\right]$

Para ilustrar o teorema, considere novamente o ideal $I=\left\langle x_{2}-x_{1}^{2}, x_{3}-x_{1}^{3}\right\rangle d a$ cúbica torcida afim $W \subset R^{3}$.

Calculando uma base de Gröebner para I com respeito a ordem grlex, temos:

$$
G=\left\{x_{1}^{2}-x_{2}, x_{1} x_{2}-x_{3}, x_{1} x_{3}-x_{2}^{2}\right\}
$$

Pelo teorema $B$, a homogenização destes polinômios gera $I^{h}$. Assim,

$$
I^{h}=<x_{1}^{2}-x_{0} x_{2}, x_{1} x_{2}-x_{0} x_{3}, x_{1} x_{3}-x_{2}^{2}>
$$

Discutiremos agora o significado geométrico da homogenização de um ideal.

Definição A.2.8 Dada uma variedade afim $W \subset K^{n}$, o fecho projetivo de $W$ é a variedade projetiva

$$
\bar{W}=V\left(I_{a}(W)^{h}\right) \subset \mathbb{P}^{n}(K)
$$

onde $I_{a}(W)^{h} \subset K\left[x_{0}, \ldots, x_{n}\right]$ é a homogenização do ideal $I_{a}(W) \subset K\left[x_{1}, \ldots, x_{n}\right]$

Proposição A.2.4 Seja $W \subset K^{n}$ uma variedade afim e seja $\bar{W} \subset \mathbb{P}^{n}(K)$ seu fecho projetivo. Então $\bar{W}$ é a menor variedade projetiva em $\mathbb{P}^{n}(K)$ contendo $W$.

Demonstração: Precisamos mostrar que se $V$ é uma variedade projetiva contendo $W$, então $\bar{W} \subset V$. 
Suponha que $V=V\left(F_{1}, \ldots, F_{s}\right)$. Então os $F_{i}$ 's se anulam em $V$, de modo que sua desomogenização $f_{i}=F_{i}\left(1, x_{1}, \ldots, x_{n}\right)$ se anula em $W$. Assim, $f_{i} \in I_{a}(W)$ e logo, $f_{i}^{h} \in I_{a}(W)^{h}$. Isto mostra que $f_{i}^{h}$ se anula em $\bar{W}=V\left(I_{a}(W)^{h}\right)$.

Mas, $F_{i}=x_{0}^{e_{i}} f_{i}^{h}$ para algum inteiro $e_{i}$.

De fato, seja $F\left(x_{0}, \ldots, x_{n}\right)$ um polinômio homogêneo de grau d e seja e a maior potência de $x_{0}$ dividindo $F$. Então $f=F\left(1, x_{1}, \ldots, x_{n}\right)$ é um polinômio de grau $(d-e)$. Assim,

$$
f^{h}=\sum_{j=0}^{d-e} F_{j}\left(1, x_{1}, \ldots, x_{n}\right) x_{0}^{(d-e)-j}, \text { onde } F_{j} \text { é o termo de grau } j \text { de } F
$$

Portanto,

$$
x_{0}^{e} f^{h}=\sum_{j=0}^{d-e} \underbrace{F_{j}\left(1, x_{1}, \ldots, x_{n}\right) x_{0}^{d-j}}_{\text {pol. hom. de grau } d}=F\left(x_{0}, x_{1}, \ldots, x_{n}\right)
$$

Assim, $F_{i}$ se anula em $\bar{W}$ e como isto é válido para todo $i$, segue que $\bar{W} \subset V$.

Vejamos agora um exemplo.

Exemplo 11 Considere a cúbica torcida afim $W \subset R^{3}$

Sabemos que $I_{a}(W)=\left\langle x_{2}-x_{1}^{2}, x_{3}-x_{1}^{3}\right\rangle$

Usando o teorema $B$, temos que

$$
I_{a}(W)^{h}=<x_{1}^{2}-x_{0} x_{2}, x_{1} x_{2}-x_{0} x_{3}, x_{1} x_{3}-x_{2}^{2}>
$$

Segue assim que a variedade

$$
V=V\left(x_{1}^{2}-x_{0} x_{2}, x_{1} x_{2}-x_{0} x_{3}, x_{1} x_{3}-x_{2}^{2}\right)
$$

é o fecho projetivo da cúbica torcida afim $W$.

O principal obstáculo da definição do fecho projetivo é que precisamos conhecer $I_{a}(W)$. Seria muito mais interessante se pudéssemos calcular o fecho diretamente de 
qualquer ideal definindo $W$. Quando $K$ é algebricamente fechado, isto sempre pode ser feito.

Teorema C: Seja $K$ um corpo algebricamente fechado, e seja $I \subset K\left[x_{1}, \ldots, x_{n}\right]$ um ideal. Então $V\left(I^{h}\right) \subset \mathbb{P}^{n}(K)$ é o fecho projetivo de $V_{a}(I) \subset K^{n}$.

Demonstraçäo: Seja $W=V_{a}(I) \subset K^{n}$ e $Z=V\left(I^{h}\right) \subset \mathbb{P}^{n}(K)$. Fazendo $Z \cap U_{0}$, teremos a porção afim de $V\left(I^{h}\right)$, ou seja, fazendo $x_{0}=1$ obtemos $V_{a}(I)=W$. Assim, temos que $Z$ é uma variedade projetiva contendo $W$.

Demonstraremos agora que $Z$ é a menor variedade projetiva contendo $W$.

Seja $V=V\left(F_{1}, \ldots, F_{s}\right)$ uma variedade projetiva qualquer contendo $W$. Temos que a desomogenização $f_{i}=F_{i}\left(1, x_{1}, \ldots, x_{n}\right) \in I_{a}(W)$. Como $K$ é algebricamente fechado, o Nullstellensatz implica que $I_{a}(W)=\sqrt{I}$, de modo que $f_{i}^{m} \in I$ para algum inteiro $m$. Isto nos diz que $\left(f_{i}^{m}\right)^{h} \in I^{h}$ e, consequentemente, $\left(f_{i}^{m}\right)^{h}$ se anula em $Z$. Mas, $\left(f_{i}^{m}\right)^{h}=\left(f_{i}^{h}\right)^{m}$, e segue que $f_{i}^{h}$ se anula em $Z$. Então $F_{i}=x_{0}^{e_{i}} f_{i}^{h}$ também se anula em $Z$. Donde concluímos que $Z \subset W$. Isto mostra que $Z$ é a menor variedade projetiva contendo $W$. Como o fecho projetivo $\bar{W}$ possui a mesma propriedade, segue que $Z=\bar{W}$.

Se combinarmos os teoremas $B$ e $C$, teremos um algorítmo para calcular o fecho projetivo de uma variedade afim sobre um corpo algebricamente fechado:

Dado $W \subset K^{n}$ definida por $f_{1}=\ldots=f_{s}=0$, calculamos uma base de Gröebner $G$ de $\left\langle f_{1}, \ldots, f_{s}\right\rangle$ com respeito a uma ordem graduada, e então o fecho projetivo em $\mathbb{P}^{n}(K)$ é definido por $V\left(G^{h}\right)$ 


\section{A.3 A Geometria das Hipersuperfícies Quádricas}

Definição A.3.1 Uma variedade $V=V(f) \subset \mathbb{P}^{n}(K)$, onde $f$ é um polinômio homogêneo não nulo de grau 2, é chamada hipersuperfície quádrica, ou simplesmente quádrica

Definição A.3.2 Dadas $V$ e $W$ duas variedades projetivas em $\mathbb{P}^{n}(K)$, dizemos que $V$ é projetivamente equivalente a $W$ se existir uma matriz invertível $A \in G L(n+1, K)$ tal que $V=A(W)$.

Observação A.3.1 Através dos resultados de álgebra linear, sabemos que as equações que definem uma quádrica são equivalentes à uma equação da forma $c_{0} x_{0}^{2}+\ldots+c_{n} x_{n}^{2}$, e se $K$ for algebricamente fechado, então a hipersuperfície quádrica de rank $p+1$ é projetivamente equivalente à quádrica definida pela equação $x_{0}^{2}+\ldots+x_{p}^{2}=0$.

Discutiremos a seguir algumas propriedades interessantes de quádricas não singulares em $\mathbb{P}^{2}, \mathbb{P}^{3}$ e $\mathbb{P}^{5}$.

Para o caso de $\mathbb{P}^{2}$ considere a aplicação $F: \mathbb{P}^{1} \rightarrow \mathbb{P}^{2}$ definida por $F(u, v)=$ $\left(u^{2}, u v, v^{2}\right)$, onde $(u, v)$ são coordenadas homogêneas em $\mathbb{P}^{1}$. Observe que os pontos de $F\left(\mathbb{P}^{1}\right)$ satisfazem a equação $x_{0} x_{2}-x_{1}^{2}=0$, mais ainda, a aplicação $F: \mathbb{P}^{1} \rightarrow$ $V\left(x_{0} x_{2}-x_{1}^{2}\right)$ é uma bijeção, de modo que esta cônica é uma cópia de $\mathbb{P}^{1}$ em $\mathbb{P}^{2}$. De fato, mostremos que $F$ é sobre:

Seja $\left(w_{0}, w_{1}, w_{2}\right) \in V\left(x_{0} x_{2}-x_{1}^{2}\right)$, logo $w_{0} w_{2}-w_{1}^{2}=0$. Sem perda de generalidade, podemos supor que $w_{0} \neq 0$, então $\left(w_{0}, w_{1}\right)$ é um ponto de $\mathbb{P}^{1} . F\left(w_{0}, w_{1}\right)=$ $\left(w_{0}^{2}, w_{0} w_{1}, w_{1}^{2}\right)=\left(w_{0}^{2}, w_{0} w_{1}, w_{0} w_{2}\right)=\left(w_{0}, w_{1}, w_{2}\right)$ 
$F$ é injetora. De fato, basta mostrarmos que todo ponto $(u, v) \in \mathbb{P}^{1}$ tal que $F(u, v)=\left(w_{0}, w_{1}, w_{2}\right) \in V$, é equivalente ao ponto $\left(w_{0}, w_{1}\right)$, pois como vimos anteriormente $F(u, v)=\left(w_{0}, w_{1}, w_{2}\right)=F\left(w_{0}, w_{1}\right)$. Logo, $F(u, v)=\left(u^{2}, u v, v^{2}\right)=$ $\lambda\left(w_{0}^{2}, w_{0} w_{1}, w_{1}^{2}\right)$. Como $w_{0} \neq 0, \lambda \neq 0$ segue que $u^{2}=\lambda w_{0} \Rightarrow u \neq 0$. Assim, $u u=$ $\lambda w_{0} w_{0} \Rightarrow u=\frac{\lambda w_{0}}{u} w_{0}$. Seja $\beta=\frac{\lambda w_{0}}{u}$. Análogamente, temos $u v=\lambda w_{0} w_{1} \Rightarrow v=\beta w_{1}$. Logo $(u, v)=\beta\left(w_{0}, w_{1}\right) \Rightarrow(u, v) \sim\left(w_{0}, w_{1}\right)$ em $\mathbb{P}^{1}$.

Quando vemos quádricas em $\mathbb{P}^{3}$ a situação é mais interessante. Considere a aplicação $F: \mathbb{P}^{1} \times \mathbb{P}^{1} \rightarrow \mathbb{P}^{3}$ que leva um ponto $\left(x_{0}, x_{1}, y_{0}, y_{1}\right) \in \mathbb{P}^{1} \times \mathbb{P}^{1}$ no ponto $\left(x_{0} y_{0}, x_{0} y_{1}, x_{1} y_{0}, x_{1} y_{1}\right) \in \mathbb{P}^{3}$. Esta aplicação é chamada aplicação de Segre.

Proposição A.3.1 A aplicação de Segre $F: \mathbb{P}^{1} \times \mathbb{P}^{1} \rightarrow \mathbb{P}^{3}$ é um-a-um e sua imagem é a quádrica não singular $V\left(z_{0} z_{3}-z_{1} z_{2}\right)$. (Note que esta é uma maneira de enxergar o toro em $\mathbb{P}^{3}$ )

Demonstração: Usaremos $\left(z_{0}, z_{1}, z_{2}, z_{3}\right)$ como coordenadas homogêneas em $\mathbb{P}^{3}$. Note que os pontos de $F\left(\mathbb{P}^{1} \times \mathbb{P}^{1}\right)$ satisfazem a equação $z_{0} z_{3}-z_{1} z_{2}=0$ assim, $F\left(\mathbb{P}^{1} \times \mathbb{P}^{1}\right) \subset$ $V\left(z_{0} z_{3}-z_{1} z_{2}\right)$. Para mostrar a igualdade, suponha $\left(w_{0}, w_{1}, w_{2}, w_{3}\right) \in V\left(z_{0} z_{3}-z_{1} z_{2}\right)$. Se $w_{0} \neq 0$, então $\left(w_{0}, w_{2}, w_{0}, w_{1}\right) \in \mathbb{P}^{1} \times \mathbb{P}^{1}$ e $F\left(w_{0}, w_{2}, w_{0}, w_{1}\right)=\left(w_{0}^{2}, w_{0} w_{1}, w_{0} w_{2}, w_{1} w_{2}\right)$. Contudo, como $w_{0} w_{3}-w_{1} w_{2}=0$, segue que:

$$
F\left(w_{0}, w_{2}, w_{0}, w_{1}\right)=\left(w_{0}^{2}, w_{0} w_{1}, w_{0} w_{2}, w_{0} w_{3}\right)=\left(w_{0}, w_{1}, w_{2}, w_{3}\right)
$$

Quando uma coordenada diferente é não nula, a demonstração é similar e segue que $F\left(\mathbb{P}^{1} \times \mathbb{P}^{1}\right)=V\left(z_{0} z_{3}-z_{1} z_{2}\right)$.

$F$ é injetiva. De fato, sem perda de generalidade, podemos supor $w_{0} \neq 0$. Basta mostrarmos que todo ponto $\left(u_{0}, u_{1}, u_{2}, u_{3}\right) \in \mathbb{P}^{1} \times \mathbb{P}^{1}$ tal que $F\left(u_{0}, u_{1}, u_{2}, u_{3}\right)=$ $\left(w_{0}, w_{1}, w_{2}, w_{3}\right) \in V$ é equivalente ao ponto $\left(w_{0}, w_{2}, w_{0}, w_{1}\right) \in \mathbb{P}^{1} \times \mathbb{P}^{1}$, pois como vimos na parte anterior da demonstração, $F\left(w_{0}, w_{2}, w_{0}, w_{1}\right)=\left(w_{0}, w_{1}, w_{2}, w_{3}\right)=$ 
$F\left(u_{0}, u_{1}, u_{2}, u_{3}\right)$. Assim, devemos mostrar que $\left(w_{0}, w_{2}, w_{0}, w_{1}\right) \sim\left(u_{0}, u_{1}, u_{2}, u_{3}\right)$ em $\mathbb{P}^{1} \times \mathbb{P}^{1}$, ou seja, $\left(w_{0}, w_{2}\right) \sim\left(u_{0}, u_{1}\right)$ e $\left(w_{0}, w_{1}\right) \sim\left(u_{2}, u_{3}\right)$ em $\mathbb{P}^{1}$. Aplicando $F$ temos que $\left(u_{0} u_{2}, u_{0} u_{3}, u_{1} u_{2}, u_{1} u_{3}\right)=\lambda\left(w_{0}^{2}, w_{0} w_{1}, w_{0} w_{2}, w_{1} w_{2}\right), u_{0} u_{2}=\lambda w_{0}^{2}$, como $w_{0} \neq 0 \Rightarrow u_{0} \neq 0$ e $u_{2} \neq 0 \Rightarrow u_{0}=\frac{\lambda w_{0}}{u_{2}} w_{0}$, seja $\alpha=\frac{\lambda w_{0}}{u_{2}} \Rightarrow u_{0}=\alpha w_{0}$. $u_{1} u_{2}=\lambda w_{0} w_{2} \Rightarrow u_{1}=\frac{\lambda w_{0}}{u_{2}} w_{2} \Rightarrow u_{1}=\alpha w_{2}$. Assim, $\left(u_{0}, u_{1}\right) \sim\left(w_{0}, w_{2}\right)$. Por outro lado, temos $u_{0} u_{2}=\lambda w_{0} w_{0} \Rightarrow u_{2}=\frac{\lambda w_{0}}{u_{0}} w_{0}$, seja $\beta=\frac{\lambda w_{0}}{u_{0}} \Rightarrow u_{2}=\beta w_{0}$, $u_{0} u_{3}=\lambda w_{0} w_{1} \Rightarrow u_{3}=\frac{\lambda w_{0}}{u_{0}} w_{1} \Rightarrow u_{3}=\beta w_{1}$. Logo $\left(u_{2}, u_{3}\right) \sim\left(w_{0}, w_{1}\right)$. Portanto $F$ é injetora.

Vamos agora mostrar que o conjunto de retas projetivas em $\mathbb{P}^{3}$ se identifica com uma quádrica em $\mathbb{P}^{5}$.

Dois pontos $p \neq q$ em $\mathbb{P}^{3}$ dão vetores l.i. $p=\left(a_{0}, a_{1}, a_{2}, a_{3}\right)$ e $q=\left(b_{0}, b_{1}, b_{2}, b_{3}\right)$ em $K^{4}$. Agora considere a aplicação $F: \mathbb{P}^{1} \rightarrow \mathbb{P}^{3}$ dada por

$$
\text { ( } \nabla) F(u, v)=\left(a_{0} u-b_{0} v, a_{1} u-b_{1} v, a_{2} u-b_{2} v, a_{3} u-b_{3} v\right)
$$

Como $p$ e $q$ são linearmente independentes, $a_{0} u-b_{0} v, a_{1} u-b_{1} v, a_{2} u-b_{2} v, a_{3} u-b_{3} v$ não podem se anular simultâneamente, de modo que $E$ está definida para todo $\mathbb{P}^{1} . A$ imagem de $F$ é uma variedade $L \subset \mathbb{P}^{3}$ definida por equações lineares que determinam um plano em $K^{4}$, logo, uma reta ęm $\mathbb{P}^{3}$. Chamamos $L$ de reta projetiva determinada por $p$ e $q$.

Lembre que uma reta $L \subset \mathbb{P}^{2}$ Ié definida por uma única equação $A_{0} X_{0}+A_{1} X_{1}+$ $A_{2} X_{2}=0$. Note que $\left(A_{0}, A_{1}, \dot{A}_{2}\right)$ pode ser observado como as "coordenadas homogêneas"de $L$ e que o conjunto de todas as retas formam o espaço projetivo dual $\mathbb{P}^{2 v}$.

Faz sentido questionarmos a thesma coisa para $\mathbb{P}^{3}$. Em particular, podemos encontrar "coordenadas homogêneas"para retas em $\mathbb{P}^{3}$ ? 
Sabemos que uma reta $L \subset \mathbb{P}^{3}$ pode ser parametrizada projetivamente usando dois pontos $p=\left(a_{0}, a_{1}, a_{2}, a_{3}\right), q=\left(b_{0}, b_{1}, b_{2}, b_{3}\right) \in K^{4}$. Então considere a matriz $2 \times 4$ cujas linhas são $p$ e $q$

$$
\Omega=\left(\begin{array}{llll}
a_{0} & a_{1} & a_{2} & a_{3} \\
b_{0} & b_{1} & b_{2} & b_{3}
\end{array}\right)
$$

Numerando as colunas de $\Omega$ usando $0,1,2,3$, então o determinante formado usando as colunas $i$ e $j$ será denotado $w_{i j}$. Podemos assumir $0 \leq i<j \leq 3$ e obtemos os seis determinantes

$$
\left(\nabla^{\prime}\right)=\left\{\begin{array}{l}
w_{01}=a_{0} b_{1}-a_{1} b_{0} \\
w_{02}=a_{0} b_{2}-a_{2} b_{0} \\
w_{03}=a_{0} b_{3}-a_{3} b_{0} \\
w_{12}=a_{1} b_{2}-a_{2} b_{1} \\
w_{13}=a_{1} b_{3}-a_{3} b_{1} \\
w_{23}=a_{2} b_{3}-a_{3} b_{2}
\end{array}\right.
$$

Usaremos a notação

$$
w(p, q)=\left(w_{01}, w_{02}, w_{03}, w_{12}, w_{13}, w_{23}\right) \in K^{6}
$$

Os $w_{i j}$ são são chamados as coordenadas de Plücker da reta L. Note que toda reta possui pelo menos uma coordenada de Plücker não nula, devido ao fato de $\Omega$ possuir rank 2. As coordenadas de Plücker de uma reta $L$ não dependem dos pontos $p$ e $q$ escolhidos. De fato, suponha que escolhemos um par diferente $q^{\prime}, p^{\prime} \in L$. Em termos de coordanadas homogêneas, $L$ pode ser descrito como o conjunto $L=\{u p-$ $\left.v q ;(u, v) \in \mathbb{P}^{1}\right\}$. Em particular, podemos escrever

$$
\begin{aligned}
& p^{\prime}=u p-v q \\
& q^{\prime}=s p-t q
\end{aligned}
$$

para pontos distintos $(u, v),(s, t) \in \mathbb{P}^{1}$. Note que

$$
\left(\begin{array}{c}
u p-v q \\
s p-t q
\end{array}\right)=\left(\begin{array}{ll}
u & -v \\
s & -t
\end{array}\right)=\left(\begin{array}{l}
p \\
q
\end{array}\right)
$$


Assim, $w(p, q)=w(u p-v q, s p-t q)=(s v-u t) w(p, q)$ em $K^{6}$, onde $s v-u t \neq 0$ pois $(u, v)$ e $(s, t)$ são pontos distintos de $\mathbb{P}^{1}$. Isto mostra que $w(p, q)$ nos dá um ponto em $\mathbb{P}^{5}$ o qual depende apenas de $L$. Assim, uma reta $L$ determina um ponto bem definido $w(L) \in \mathbb{P}^{5}$.

Variando $L$ sobre as retas em $\mathbb{P}^{3}$, as coordenadas de Plücker $w(L)$ irão descrever um certo subconjunto de $\mathbb{P}^{5}$. Eliminando os $a_{i}^{\prime} s$ e $b_{i}^{\prime} s$ de $\left(\nabla^{\prime}\right)$, é fácil ver que $w_{01} w_{23}-$ $w_{02} w_{13}+w_{03} w_{12}=0$ para todos os conjuntos de coordenadas de Plücker. Considerando $z_{i j}, 0 \leq i<j \leq 3$, coordenadas homogêneas em $\mathbb{P}^{5}$, segue que os pontos de $w(L)$ pertencem à quádrica não sigular $V\left(z_{01} z_{23}-z_{02} z_{13}+z_{03} z_{12}\right) \subset \mathbb{P}^{5}$.

Teorema A.3.1 A aplicação $\left\{\right.$ retas em $\left.\mathbb{P}^{3}\right\} \rightarrow V\left(z_{01} z_{23}-z_{02} z_{13}+z_{03} z_{12}\right)$ definida por enviar uma reta $L \subset \mathbb{P}^{3}$ em suas coordenadas de Plücker $w(L) \in V\left(z_{01} z_{23}-\right.$ $\left.z_{02} z_{13}+z_{03} z_{12}\right) \in \mathbb{P}^{5}$ é uma bijeção.

Demonstração: A estratégia da demonstração é mostrar que uma reta $L \subset \mathbb{P}^{3}$ pode ser reconstruída pelas suas coordenadas de Plücker. Dados dois pontos $p=$ $\left(a_{0}, a_{1}, a_{2}, a_{3}\right)$ e $q=\left(b_{0}, b_{1}, b_{2}, b_{3}\right)$ em $L$, é fácil checar que conseguimos os quatro vetores em $K^{4}$

$$
\text { (*) } \begin{aligned}
b_{0} p-a_{0} q & =\left(0,-w_{01},-w_{02},-w_{03}\right) \\
b_{1} p-a_{1} q & =\left(w_{01}, 0,-w_{12},-w_{13}\right) \\
b_{2} p-a_{2} q & =\left(w_{02}, w_{12}, 0,-w_{23}\right) \\
b_{3} p-a_{3} q & =\left(w_{03}, w_{13}, w_{23}, 0\right)
\end{aligned}
$$

Sempre que estes vetores forem não nulos, ele nos fornecem pontos de $L$.

Para Mostrar que w é 1-1, suponha que temos retas $L$ e $L^{\prime}$ tais que $w(L)=\lambda w\left(L^{\prime}\right)$ para algum $\lambda$ não nulo. Em termos das coordenadas de Plücker, isto significa que $w_{i j}=\lambda w_{i j}^{\prime}$ para todo $0 \leq i<j \leq 3$. Sabemos que alguma coordenada de Plücker 
de $L$ é não nula, e por mudança de coordenadas em $\mathbb{P}^{3}$, podemos assumir $w_{01} \neq 0$. Então (*) implica que em $\mathbb{P}^{3}$, os pontos

$$
\begin{aligned}
& P=\left(0,-w_{01}^{\prime},-w_{02}^{\prime},-w_{03}^{\prime}\right)=\left(0,-\lambda w_{01},-\lambda w_{02},-\lambda w_{03}\right)=\left(0,-w_{01},-w_{02},-w_{03}\right) \\
& Q=\left(w_{01}^{\prime}, 0,-w_{12}^{\prime},-w_{13}^{\prime}\right)=\left(\lambda w_{01}, 0,-\lambda w_{12},-\lambda w_{13}\right)=\left(w_{01}, 0,-w_{12},-w_{13}\right)
\end{aligned}
$$

estão ambos em $L$ e $L^{\prime}$. Como existe uma única reta passando por dois pontos em $\mathbb{P}^{3}$, segue que $L=L^{\prime}$. Isto mostra que nossa aplicação é 1-1.

Para ver que $w$ é sobre, escolha um ponto $\left(w_{01}, w_{02}, w_{03}, w_{12}, w_{23}\right) \in V\left(z_{01} z_{23}-\right.$ $\left.z_{02} z_{13}+z_{03} z_{12}\right)$. Por mudanças de coordenadas em $\mathbb{P}^{3}$, podemos assumir $w_{01} \neq 0$. Então os dois primeiros vetores de (*) são não nulos e assim, determinam uma reta $L \subset \mathbb{P}^{3}$. Vamos calcular as coordenadas de Plücker desta reta:

$$
\begin{aligned}
& w_{01}^{\prime}=w_{01}^{2} \\
& w_{02}^{\prime}=w_{01} w_{02} \\
& w_{03}^{\prime}=w_{01} w_{03} \\
& w_{12}^{\prime}=w_{01} w_{12} \\
& w_{13}^{\prime}=w_{01} w_{13} \\
& w_{23}^{\prime}=w_{02} w_{13}-w_{03} w_{12}
\end{aligned}
$$

Usando o fato de que $\left(w_{01}, w_{02}, w_{03}, w_{12}, w_{23}\right) \in V\left(z_{01} z_{23}-z_{02} z_{13}+z_{03} z_{12}\right)$, temos $q u e-w_{03} w_{12}=w_{01} w_{23}-w_{02} w_{13}$ e usando isto em $w_{23}^{\prime}$ segue:

$$
w_{23}^{\prime}=w_{01} w_{23}
$$

Assim, $w(L)=\left(w_{01}^{2}, w_{01} w_{02}, w_{01} w_{03}, w_{01} w_{12}, w_{01} w_{13}, w_{01} w_{23}\right)$, como $w_{01} \neq 0$, temos $w(L)=\left(w_{01}, w_{02}, w_{03}, w_{12}, w_{13}, w_{23}\right)$. Portanto $w_{i j}$ são as coordenadas de Plücker de $L$, logo w é sobre. 


\section{Referências Bibliográficas}

[1] J. Martinet. Singularieties of smooth functions and maps, volume 58 of London Math. Soc. Lectures Notes. Cambridge University Press, 1982.

[2] J. N. Damon. The unfolding and determinacy theorems for subgroups of $\mathcal{A}$ and K. Mem. Amer. Math. Soc., 50, 1984.

[3] C. G. Gibson. Singular points of smooth mappings, volume 25 of Pitman Research Notes in Mathematics. 1979.

[4] J. A. Montaldi. On generic composite of maps. Bull. Lond. Math. Soc, 23:81-85, 1991.

[5] J. W. Bruce, N. Kirk, and A. A. du Plessis. Complete transversals and the classifications of singularities. Nonlinearity, 10, 1997.

[6] J. N. Mather. Stability of $C^{\infty}$-mappings IV: Classification of stable map-germs by $\mathbb{R}$-algebras. Publ. Math. IHES, 37:223-248, 1970.

[7] C. T. C. Wall. Classification and stability of singularities of smooth maps. In D. T. Lê, K. Saito, and B. Tessier, editors, Singularity Theory, pages 920-952. World Scientific Publishing, 1995.

[8] J.W. Bruce, A. A. du Plessis, and C. T. C. Wall. Determinacy and unipotency. Invent. Math., 88:521-554, 1987. 
[9] J. H. Rieger. Families of maps from the plane to the plane. J. London Math. soc,36:351-369, 1987.

[10] J.W. Bruce and P.J. Giblin. Curves And singularities, Cambridge University Press.

[11] Shafarevich.Basic Algebraic Geometry 1, Varieties in projective space, Springer Verlag(Teorema 3, página 58).

[12] Farid Tari. Singularidades de aplicasōoes diferenciáveis, Notas Didáticas do ICMC-USP.

[13] J.W.Bruce. The duals of generic hypersurfaces, Math. Scand.49(1981), 36-60.

[14] D.Mumford. Algebraic Geometry I: Complex projective varieties, Springer Verlag, Berlin-Heidelberg-New York, 1976.

[15] David Cox, John Little and Donal O'Shea;Ideals, varieties, and algorithms - An Introduction to Computational Algebraic Geometry and Commutative Algebra; Springer-Verlag.Springer-Verlag.

[16] J.W.Bruce, Generic geometry and duality, LMS Lecture Note Series 201, Edited by J.P.Brasselet(1994)pp 29-60].

[17] Clint McCrory and Theodore Shifrin, Cusps of the projective Gauss map, J. Differential Geometry 19(1984)257-276.

[18] A. Nogueira. Superfícies em $\mathbb{R}^{4}$ e dualidade. Tese de doutorado, Instituto de Ciências Matemáticas e de Computação, USP-São Carlos, 1998.

[19] E.J.N.Looijenga. Structural stability of smooth families of $C^{\infty}$ functions, Thesis, University of Amsterdam, 1974. 
[20] C.T.C.Wall, Geometric properties of generic differentiable manifolds, in: Geometry and Topology III(Lecture Notes in Mathematics 597), Springer-Verlag, Berlin - Heidelberg - New York, 1976.

[21] T.Banchoff, T.Gaffney, C.McCrory, Cusps of Gauss mappings, Research Notes in Mathematics, Pitman Advanced Publishing Program, Boston - London - Melbourne.

[22] E.Bierstone, D.Milman, Semianalytic and subanalytic sets, I.H.E.S - Publications Mathématiques, 67,1988.

[23] Hideyuki Matsumura, Commutative algebra, W.A.Benjamin, Inc, New York 1970.

[24] William Fulton, Algebraic curves. An introduction to algebraic geometry, Mathematics Lecture Note Series.

[25] Israel Vainsencher, Introdução às curvas algébricas planas, Décimo segundo colóquio brasileiro de matemática, IMPA, 1979. 